\title{
Stereoselective Total Synthesis of Bioactive Marine Natural Product Biselyngbyolide B
}

\author{
Sayantan Das, Debobrata Paul and Rajib Kumar Goswami* \\ Department of Organic Chemistry, \\ Indian Association for the Cultivation of Science, Jadavpur, \\ Kolkata-700032, India. \\ Email: ocrkg@iacs.res.in
}

Supporting Information

1. General Experimental Procedure: S2

2. ${ }^{1} \mathrm{H}$ and ${ }^{13} \mathrm{C}$-NMR Comparison of Natural Biselyngbyolide B with Synthetic Biselyngbyolide B: S3

3. 2D-NMR Correlation and Structure Confirmation of Macrocycle (27) of Biselyngbyolide B: S4

4. Experimental Procedure: S5-S23

5. Copies of ${ }^{1} \mathrm{H}-\mathrm{NMR},{ }^{13} \mathrm{C}-\mathrm{NMR}, 2 \mathrm{D}-\mathrm{NMR}$ and HRMS Spectra: S24-S44 


\section{General Experimental Procedure:}

All moisture sensitive reactions were performed in oven or flame-dried glassware with Teflon coated magnetic stirring bar under argon atmosphere using dry, freshly distilled solvents, unless otherwise noted. Air- and moisture-sensitive liquids were transferred via a gastight syringe and a stainless-steel needle. Reactions were monitored by thin layer chromatography (TLC, Silica gel $60 \mathrm{~F}_{254}$ ) plates with UV light, ethanolic anisaldehyde (with $1 \% \mathrm{AcOH}$ and $3.3 \%$ conc. $\mathrm{H}_{2} \mathrm{SO}_{4}$ )heat and aqueous $\mathrm{KMnO}_{4}$ (with $\mathrm{K}_{2} \mathrm{CO}_{3}$ and $10 \%$ aqueous $\mathrm{NaOH}$ solution) as developing agents. All workup and purification procedures were carried out with reagent-grade solvents under ambient atmosphere unless otherwise stated. Column chromatography was performed using silica gel 60-120 mesh, 100-200 mesh and 230-400 mesh. Yields mentioned as chromatographically and spectroscopically homogeneous materials unless otherwise stated. Optical rotations were measured only for pure compounds and not for mixtures using sodium (589, D line) lamp and are reported as follows: $[\alpha]^{25} \mathrm{D}(c=\mathrm{mg} / 100 \mathrm{~mL}$, solvent). IR spectra were recorded as thin films (for liquids). HRMS were taken using Quadruple-TOF (Q-TOF) micro MS system using electrospray ionization (ESI) technique. ${ }^{1} \mathrm{H}$ NMR spectra were recorded on 300 , 400 and $500 \mathrm{MHz}$ spectrometers in appropriate solvents and calibrated using residual undeuterated solvent as an internal reference, and the chemical shifts are shown in $\delta$ ppm scales. Multiplicities of NMR signals are designated as s (singlet), $\mathrm{d}$ (doublet), $\mathrm{t}$ (triplet), q (quartet), br

(broad), m (multiplet, for unresolved lines) etc. ${ }^{13} \mathrm{C}$ and 2D NMR spectra were recorded on 75 , 100 and $125 \mathrm{MHz}$ spectrometers. 
2. Table 1: ${ }^{1} \mathrm{H}$ and ${ }^{13} \mathrm{C}$ NMR comparison of natural Biselyngbyolide $\mathrm{B}$ with synthetic Biselyngbyolide $\mathrm{B}$

\begin{tabular}{|c|c|c|c|c|}
\hline \multicolumn{2}{|c|}{$\boldsymbol{\delta}_{\mathbf{H}}$ (Multiplicity, $J$ in $\mathrm{Hz}$ ) } & \multicolumn{3}{|c|}{$\boldsymbol{\delta}_{\mathrm{C}}$} \\
\hline \multirow{2}{*}{$\begin{array}{c}\text { Natural } \\
\mathrm{C}_{6} \mathrm{D}_{6}(400 \mathrm{MHz})\end{array}$} & \multirow{2}{*}{$\begin{array}{c}\text { Synthetic } \\
\mathrm{C}_{6} \mathrm{D}_{6}(300 \mathrm{MHz})\end{array}$} & \multirow{2}{*}{$\begin{array}{c}\text { Natural } \\
\mathrm{C}_{6} \mathrm{D}_{6}(100 \mathrm{MHz})\end{array}$} & \multicolumn{2}{|c|}{ Synthetic } \\
\hline & & & $\mathrm{C}_{6} \mathrm{D}_{6}(125 \mathrm{MHz})$ & $\mathrm{CDCl}_{3}(75 \mathrm{MHz})$ \\
\hline $6.05(\mathrm{dd}, 15.2,10.8,1 \mathrm{H})$ & $6.05(\mathrm{dd}, 14.6,10.5,1 \mathrm{H})$ & 171.6 & 171.1 & 172.9 \\
\hline $5.96(\mathrm{dd}, 15.2,10.8,1 \mathrm{H})$ & $5.96(\mathrm{dd}, 14.6,10.5,1 \mathrm{H})$ & 139.6 & 139.4 & 140.1 \\
\hline $5.87(\mathrm{dt}, 9.6,4.8,1 \mathrm{H})$ & $5.87(\mathrm{dt}, 9.3,3.6,1 \mathrm{H})$ & 136.5 & 136.3 & 137.1 \\
\hline \multirow{5}{*}{$\begin{array}{l}5.44(\mathrm{~m}, 2 \mathrm{H}) \\
5.43(\mathrm{~m}, 1 \mathrm{H}) \\
5.40(\mathrm{~m}, 1 \mathrm{H}) \\
5.37(\mathrm{~m}, 1 \mathrm{H}) \\
5.34(\mathrm{~m}, 1 \mathrm{H})\end{array}$} & \multirow[t]{5}{*}{$5.49-5.32(\mathrm{~m}, 6 \mathrm{H})$} & 134.8 & 134.2 & 134.5 \\
\hline & & 133.8 & 133.4 & 133.6 \\
\hline & & 133.3 & 133.0 & 132.8 \\
\hline & & 132.6 & 132.9 & 131.9 \\
\hline & & 130.8 & 130.5 & 130.4 \\
\hline $5.28(\mathrm{~m}, 1 \mathrm{H})$ & $5.28(\mathrm{~d}, 9.0,1 \mathrm{H})$ & 129.0 & 128.8 & 129.6 \\
\hline $4.87(\mathrm{brd}, 10.0,1 \mathrm{H})$ & $4.88(\mathrm{dd}, 9.9,1 \mathrm{H})$ & 128.4 & $\underset{\text { Mignal }}{\text { Merged in } \mathrm{C}_{6} \mathrm{D}_{6}}$ & 128.0 \\
\hline $\begin{array}{c}4.48(\mathrm{ddd}, 9.6,6.4,3.2 \\
1 \mathrm{H})\end{array}$ & $4.51-4.45(\mathrm{~m}, 1 \mathrm{H})$ & 127.0 & $\begin{array}{c}\text { Merged in } \mathrm{C}_{6} \mathrm{D}_{6} \\
\text { signal }\end{array}$ & 126.7 \\
\hline $3.35(\mathrm{dd}, 10.8,4.8,1 \mathrm{H})$ & $3.35(\mathrm{dd}, 10.5,5.1,1 \mathrm{H})$ & 126.6 & 126.2 & 126.6 \\
\hline $3.08(\mathrm{~s}, 3 \mathrm{H})$ & $3.08(\mathrm{~s}, 3 \mathrm{H})$ & 124.5 & 124.2 & 123.6 \\
\hline $3.03(\mathrm{~m}, 1 \mathrm{H})$ & $3.05-2.99(\mathrm{~m}, 1 \mathrm{H})$ & 88.1 & 87.8 & 87.8 \\
\hline $2.77(\mathrm{~m}, 1 \mathrm{H})$ & $2.80-2.74(\mathrm{~m}, 1 \mathrm{H})$ & 71.4 & 71.1 & 71.3 \\
\hline \multirow{5}{*}{$\begin{array}{l}2.39(\mathrm{~m}, 1 \mathrm{H}) \\
2.38(\mathrm{~m}, 1 \mathrm{H}) \\
2.36(\mathrm{~m}, 2 \mathrm{H}) \\
2.28(\mathrm{~m}, 1 \mathrm{H}) \\
2.26(\mathrm{~m}, 2 \mathrm{H})\end{array}$} & \multirow[t]{5}{*}{$2.39-2.21(\mathrm{~m}, 7 \mathrm{H})$} & 70.1 & 69.7 & 70.3 \\
\hline & & 55.2 & 54.9 & 55.5 \\
\hline & & 43.7 & 43.5 & 43.2 \\
\hline & & 40.9 & 40.6 & 40.8 \\
\hline & & 39.9 & 39.6 & 39.5 \\
\hline $2.10(\mathrm{~m}, 1 \mathrm{H})$ & $2.15-2.02(\mathrm{~m}, 1 \mathrm{H})$ & 36.2 & 36.1 & 35.9 \\
\hline $1.77(\mathrm{~m}, 1 \mathrm{H})$ & $1.83-1.72(\mathrm{~m}, 1 \mathrm{H})$ & 35.5 & 35.2 & 34.9 \\
\hline $1.62(\mathrm{~d}, 1.2,3 \mathrm{H})$ & $1.62(\mathrm{~d}, 1.2,3 \mathrm{H})$ & 33.2 & 33.0 & 33.2 \\
\hline $1.56(\mathrm{~m}, 3 \mathrm{H})$ & $1.56(\mathrm{~m}, 3 \mathrm{H})$ & 23.5 & 23.3 & 23.5 \\
\hline $1.51(\mathrm{~d}, 1.2,3 \mathrm{H})$ & $1.51(\mathrm{~d}, 1.2,3 \mathrm{H})$ & 22.4 & 22.7 & 22.5 \\
\hline \multirow[t]{2}{*}{$0.87(\mathrm{~d}, 6.8,3 \mathrm{H})$} & \multirow[t]{2}{*}{$0.88(\mathrm{~d}, 6.8,3 \mathrm{H})$} & 18.0 & 17.9 & 17.9 \\
\hline & & 10.0 & 9.7 & 9.9 \\
\hline
\end{tabular}


3. Figure 1: Key 2D-NMR Correlation and Structure Confirmation of Macrocycle 27 of Biselyngbyolide B

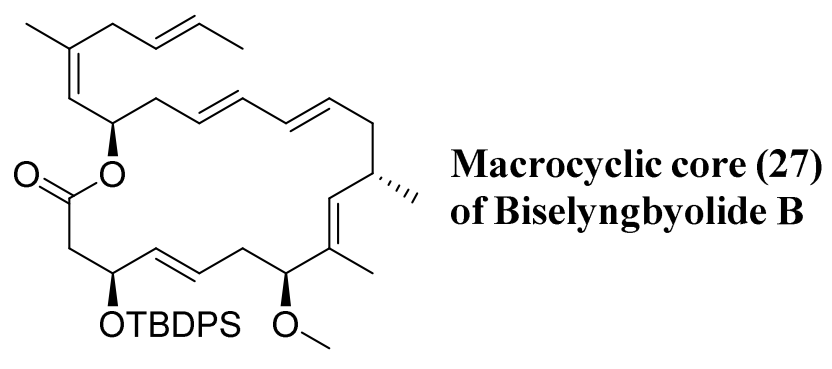

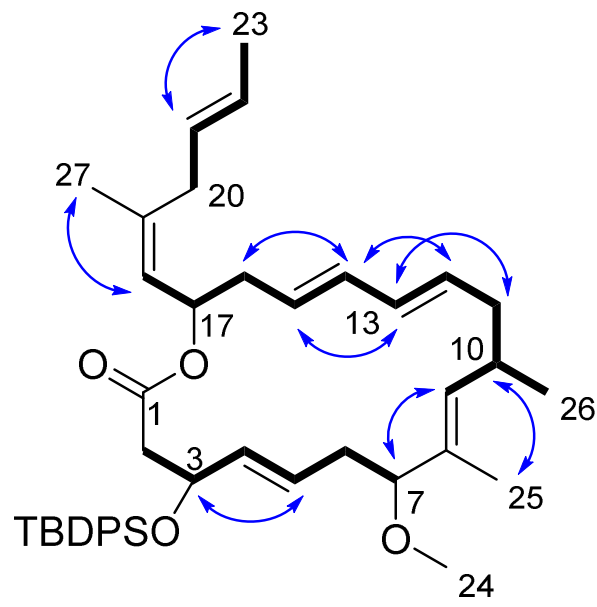

- ${ }^{1} \mathrm{H}-{ }^{1} \mathrm{H}$ COSY correlation

Key NOESY correlation

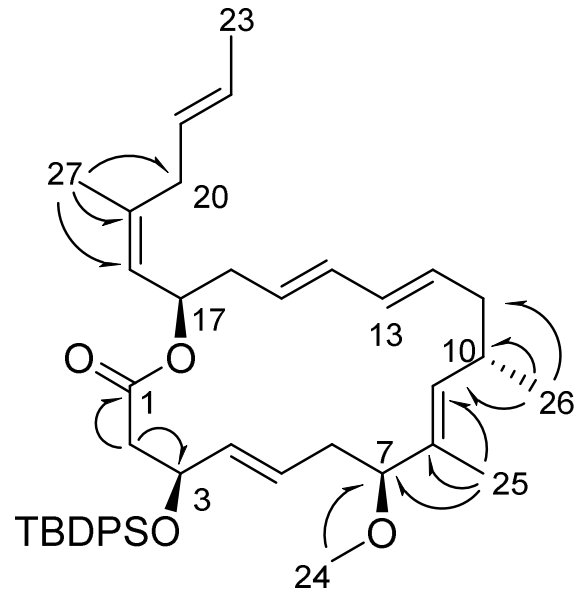

$\overbrace{{ }^{1} \mathrm{H}} \mathrm{HMBC}$ correlation 


\section{Experimental Procedure:}

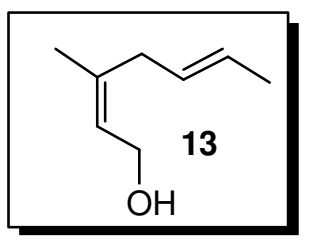

(2Z, 5E)-3-methylhepta-2, 5-dien-1-ol (13): 2-butyn-1-ol 12 (155 mg, 2.2 mmol, dissolved in $2 \mathrm{~mL}$ dry $\mathrm{Et}_{2} \mathrm{O}$ ) was added drop wise to a freshly dried $\mathrm{Et}_{2} \mathrm{O}(10 \mathrm{~mL})$ solution of Red-Al (65 wt. \% in toluene, $\left.1.5 \mathrm{~mL}, 5.1 \mathrm{mmol}\right)$ at $0{ }^{\circ} \mathrm{C}$. The reaction mixture was warmed slowly to room temperature and stirred further for $6 \mathrm{~h}$. The reaction mixture was again cooled to $0{ }^{\circ} \mathrm{C}$ and subsequently crotyl bromide 12a $(0.7 \mathrm{~mL}, 6.6$ mmol) was added and the reaction was continued further for $2 \mathrm{~h}$ at the same temperature before quenching with a saturated solution of sodium potassium tartrate $(5 \mathrm{~mL})$. The resultant mixture was extracted with EtOAc $(2 \times 25 \mathrm{~mL})$, washed with brine, dried $\left(\mathrm{Na}_{2} \mathrm{SO}_{4}\right)$, filtered and concentrated in vacuo. Purification by column chromatography $\left(\mathrm{SiO}_{2}, 100-200\right.$ mesh, $12 \%$ EtOAc in hexane as eluant) furnished major product $\mathbf{1 3}$ with an inseparable mixture of its regioisomer 13a $(198 \mathrm{mg}, 71 \%, \mathbf{1 3} / \mathbf{1 3 a}=2.3: 1)$ as a pale yellow liquid: $R_{f}=0.31(10 \%$ EtOAc in hexane); ${ }^{1} \mathrm{H} \mathrm{NMR}\left(\mathrm{CDCl}_{3}, 300 \mathrm{MHz}\right) \delta 5.47-5.31(\mathrm{~m}, 3 \mathrm{H}), 4.12(\mathrm{~d}, J=6.9 \mathrm{~Hz}, 2 \mathrm{H}), 2.74(\mathrm{~d}, J=$ $6.0 \mathrm{~Hz}, 2 \mathrm{H}), 1.72(\mathrm{~s}, 3 \mathrm{H}), 1.66-1.64(\mathrm{~m}, 3 \mathrm{H}) ;{ }^{13} \mathrm{C} \mathrm{NMR}\left(\mathrm{CDCl}_{3}, 75 \mathrm{MHz}\right) \delta 139.0,128.4,126.3$, 124.4, 59.1, 35.3, 23.6, $17.9 \mathrm{ppm}$; IR(neat): $v_{\max } 3353,2925,1001 \mathrm{~cm}^{-1}$;

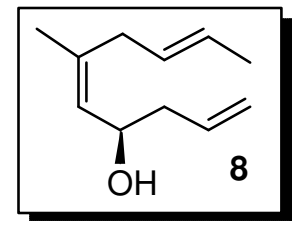

$(R, 5 Z, 8 E)$-6-methyldeca-1, 5, 8-trien-4-ol (8): To an ice cold solution of the mixture of alcohols $13 \& \mathbf{1 3 a}(120 \mathrm{mg}, 0.95 \mathrm{mmol})$ in dry $\mathrm{CH}_{2} \mathrm{Cl}_{2}(5$ $\mathrm{mL})$, DMP (807 mg, $1.90 \mathrm{mmol})$ was added under argon atmosphere. 
The reaction was stirred for 45 min prior to quench it with mixture of saturated aqueous solution of $\mathrm{Na}_{2} \mathrm{~S}_{2} \mathrm{O}_{3}$ and $\mathrm{NaHCO}_{3}(1: 1,3 \mathrm{~mL})$. The resultant mixture was stirred vigorously for $2 \mathrm{~h}$ and extracted with $\mathrm{Et}_{2} \mathrm{O}(2 \times 15 \mathrm{~mL})$, washed with water, brine, dried $\left(\mathrm{Na}_{2} \mathrm{SO}_{4}\right)$ and concentrated in vасио. Careful purification by column chromatography $\left(\mathrm{SiO}_{2}, 230-400\right.$ mesh, 2\% EtOAc in hexane eluant) afforded pure aldehyde $\mathbf{1 4}$ as a colorless liquid $\left(\mathrm{R}_{f}=0.78,10 \%\right.$ EtOAc in hexane $)$ which subsequently was taken in next step without further characterizations.

The solution of the above aldehyde $\mathbf{1 4}$ in anhydrous $\mathrm{Et}_{2} \mathrm{O}(2 \mathrm{~mL})$ was added drop wise to anhydrous $\mathrm{Et}_{2} \mathrm{O}$ solution $(4 \mathrm{~mL})$ of $(+) \mathrm{Ipc}_{2} \mathrm{~B}($ allyl $)$ borane $(1.0 \mathrm{M}$ in dioxane, $1.9 \mathrm{~mL}, 1.9 \mathrm{mmol})$ at $-78{ }^{\circ} \mathrm{C}$. The reaction mixture was stirred for $4 \mathrm{~h}$ at the same temperature prior to dilute it with $30 \%$ aqueous $\mathrm{H}_{2} \mathrm{O}_{2}(2 \mathrm{~mL})$ and $3 \mathrm{M}$ aqueous $\mathrm{NaOH}(4 \mathrm{~mL})$. The resultant mixture was stirred further for $2 \mathrm{~h}$ at room temperature and extracted with EtOAc $(3 \times 15 \mathrm{~mL})$, washed with brine, dried $\left(\mathrm{Na}_{2} \mathrm{SO}_{4}\right)$, filtered, and concentrated. The residual oil was carefully purified by column chromatography $\left(\mathrm{SiO}_{2}, 100-200\right.$ mesh, 15\% EtOAc in hexane as eluant) to give required alcohol 8 (together with ent-8, $122 \mathrm{mg}, 77 \%$ over 2 steps, ee $=80 \%$, confirmed from modified Mosher ester analysis in the next stage) as a colorless oil: $R_{f}=0.36\left(15 \%\right.$ EtOAc in hexane); ${ }^{1} \mathrm{H}$ NMR $\left(\mathrm{CDCl}_{3}, 300 \mathrm{MHz}\right) \delta 5.88-5.74(\mathrm{~m}, 1 \mathrm{H}), 5.51-5.31(\mathrm{~m}, 2 \mathrm{H}), 5.23(\mathrm{~d}, J=8.7 \mathrm{~Hz}, 1 \mathrm{H}), 5.16-5.10(\mathrm{~m}$, 2H), 4.46-4.38(m, 1H), 2.77-2.74(m, 2H), 2.27(t, $J=6.9 \mathrm{~Hz}, 2 \mathrm{H}), 1.70(\mathrm{~d}, J=1.2 \mathrm{~Hz}, 3 \mathrm{H})$, 1.65(dd, $J=6.0,1.2 \mathrm{~Hz}, 3 \mathrm{H}) ;{ }^{13} \mathrm{C} \mathrm{NMR}\left(\mathrm{CDCl}_{3}, 75 \mathrm{MHz}\right) \delta 138.3,134.7,128.5,128.0,126.5$, 118.0, 67.6, 42.4, 35.8, 23.6, 17.9 ppm; IR(neat): $v_{\max } 3386,2926 \mathrm{~cm}^{-1}$; HRMS (ESI) $\mathrm{m} / z$ calculated for $\mathrm{C}_{11} \mathrm{H}_{18} \mathrm{ONa}[\mathrm{M}+\mathrm{Na}]^{+} 189.1255$, found 189.1256 . 


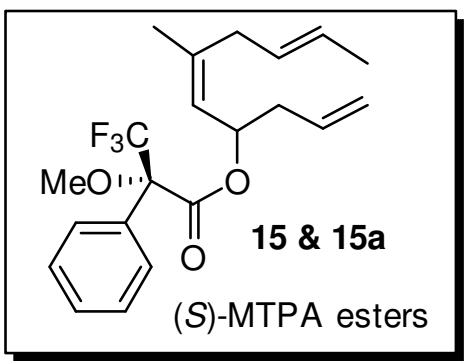

(5Z, 8E)-6-methyldeca-1,5,8-trien-4-yl $\quad(2 S)$-3,3,3-trifluoro-2methoxy-2-phenylpropanoate $(15 \&$ 15a): To a stirred solution of $(S)$-Mosher's acid $(51 \mathrm{mg}, 0.22 \mathrm{mmol})$ in anhydrous toluene (2 $\mathrm{mL})$ at room temperature, DMAP (27 mg, $0.24 \mathrm{mmol}), \mathrm{Et}_{3} \mathrm{~N}(0.03$ $\mathrm{mL}, 0.24 \mathrm{mmol})$ and 2,4,6-trichlorobenzoyl chloride $(0.03 \mathrm{~mL}, 0.22 \mathrm{mmol})$ were added sequentially under argon atmosphere and stirred for 30 minutes. A solution of alcohol 8 (together with ent-8, $12 \mathrm{mg}, 0.07 \mathrm{mmol})$ in dry toluene $(1 \mathrm{~mL})$ was then cannulated. The reaction mixture stirred further for $4 \mathrm{~h}$ at same temperature prior to quench it with saturated aqueous $\mathrm{NH}_{4} \mathrm{Cl}(1$ $\mathrm{mL})$. The resultant mixture was then extracted with EtOAc $(10 \mathrm{~mL})$, washed with water, brine, dried $\left(\mathrm{Na}_{2} \mathrm{SO}_{4}\right)$ and concentrated in vacuo. Purification of crude product by column chromatography (silica gel, 100-200mesh, 5\% EtOAc in hexane) yielded inseparable mixture of $(S)$-MTPA esters 15 and 15a (21 mg, 75\%, $d r=9: 1)$ as a colorless oil. $R_{f}=0.72(10 \%$ EtOAc in hexane); ${ }^{1} \mathrm{H}$ NMR $\left(\mathrm{CDCl}_{3}, 300 \mathrm{MHz}\right) \delta$ 7.50-7.47(m, 2H), 7.38-7.35(m, 3H), 5.80-5.66(m, $\left.2 \mathrm{H}\right)$, 5.51-5.42(m, $1 \mathrm{H}), 5.37-5.27(\mathrm{~m}, 1 \mathrm{H}), 5.13-5.07(\mathrm{~m}, 3 \mathrm{H}), 3.53(\mathrm{~s}, 3 \mathrm{H}), 2.97(\mathrm{dd}, J=14.4,6.6 \mathrm{~Hz}$ $1 \mathrm{H},), 2.37(\mathrm{dd}, J=14.4,6.6 \mathrm{~Hz}, 1 \mathrm{H}), 2.50-2.34(\mathrm{~m}, 2 \mathrm{H}), 1.68(\mathrm{~d}, J=1.5 \mathrm{~Hz}, 3 \mathrm{H}), 1.64(\mathrm{dd}, J=6.0$, $1.5 \mathrm{~Hz}, 3 \mathrm{H}) ;{ }^{13} \mathrm{C} \mathrm{NMR}\left(\mathrm{CDCl}_{3}, 75 \mathrm{MHz}\right) \delta 165.9,141.4,133.2,132.6,129.5,128.3,127.9,127.6$, $127.5,126.9,122.4,118.4,73.6,55.6,39.3,35.9,23.6,23.5,17.9$ ppm; IR(neat): $v_{\max } 2922$, 1743, $1169 \mathrm{~cm}^{-1}$; HRMS (ESI) $\mathrm{m} / z$ calculated for $\mathrm{C}_{21} \mathrm{H}_{25} \mathrm{~F}_{3} \mathrm{O}_{3} \mathrm{Na}[\mathrm{M}+\mathrm{Na}]^{+}$405.1653, found 405.1656. 


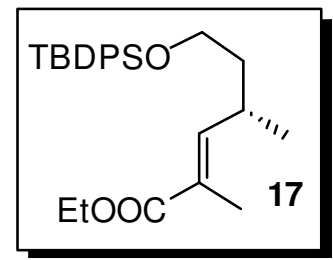

\section{$(S, E)$-Ethyl-6-((tert-butyldiphenylsilyl) oxy)-2,4-dimethylhex-2-enoate}

(17): To a stirred solution of $(\mathrm{COCl})_{2}(3.8 \mathrm{~mL}, 43.8 \mathrm{mmol})$ in anhydrous

$\mathrm{CH}_{2} \mathrm{Cl}_{2}(60 \mathrm{~mL})$ at $-78{ }^{\circ} \mathrm{C}$, DMSO $(6.6 \mathrm{~mL}, 93.4 \mathrm{mmol})$ was added drop wise under argon atmosphere. After $15 \mathrm{~min}$, alcohol $16(10 \mathrm{~g}, 29.2 \mathrm{mmol}$ dissolved in $20 \mathrm{~mL}$ anhydrous $\mathrm{CH}_{2} \mathrm{Cl}_{2}$ ) was cannulated to the reaction mixture. After $30 \min \mathrm{Et}_{3} \mathrm{~N}(20.0 \mathrm{~mL}, 146.0$ mmol) was added and the reaction was continued further for 30 min. The reaction was then warmed slowly to $0{ }^{\circ} \mathrm{C}$ and stirred further for $45 \mathrm{~min}$. The reaction mixture was quenched with saturated aqueous $\mathrm{NH}_{4} \mathrm{Cl}(25 \mathrm{~mL})$ and extracted with $\mathrm{Et}_{2} \mathrm{O}(2 \times 150 \mathrm{~mL})$, washed with saturated $\mathrm{NaHCO}_{3}$, water, brine, dried $\left(\mathrm{Na}_{2} \mathrm{SO}_{4}\right)$ and concentrated in vacuo. Flash column chromatography $\left(\mathrm{SiO}_{2}, 60-120\right.$ mesh, 3\% EtOAc in hexane as eluant) of the crude residue provided the corresponding aldehyde as a colorless oil $\left[R_{f}=0.53\right.$ (5\% EtOAc in hexane) $]$ which was taken forward without further characterizations.

To a solution of above aldehyde in anhydrous toluene $(50 \mathrm{~mL})$ at $80{ }^{\circ} \mathrm{C}$ under argon, $\mathrm{Ph}_{3} \mathrm{P}=\mathrm{CH}(\mathrm{Me}) \mathrm{CO}_{2} \mathrm{Et}(21.2 \mathrm{~g}, 58.4 \mathrm{mmol})$ was added at once. The reaction was continued for 24 $\mathrm{h}$ at the same temperature. The reaction mixture was cooled to room temperature and concentrated under reduced pressure. The residue obtained was purified by flash chromatography ( $\mathrm{SiO}_{2}, 230-400$ mesh, 2\% EtOAc in hexane as eluant) to afford ester 17 (9.8 g, 79\% over 2 steps) as a colorless oil: $\mathrm{R}_{f}=0.56\left(5 \%\right.$ EtOAc in hexane); $[\alpha]_{\mathrm{D}}{ }^{29}=+13.7\left(\right.$ c $\left.1.89 \mathrm{CHCl}_{3}\right) ;{ }^{1} \mathrm{H}$ NMR $\left(\mathrm{CDCl}_{3}, 300 \mathrm{MHz}\right) \delta$ 7.65-7.61(m, 4H), 7.43-7.32(m, 6H), 6.51(dd, $\left.J=9.9,1.2 \mathrm{~Hz}, 1 \mathrm{H}\right), 4.17(\mathrm{q}$, $J=7.2 \mathrm{~Hz}, 2 \mathrm{H}), 3.65-3.59(\mathrm{~m}, 2 \mathrm{H})$, 
$2.81-2.75(\mathrm{~m}, 1 \mathrm{H}), 1.83(\mathrm{~d}, J=1.5 \mathrm{~Hz}, 3 \mathrm{H}), 1.65-1.49(\mathrm{~m}, 2 \mathrm{H}), 1.28(\mathrm{t}, J=7.2 \mathrm{~Hz}, 3 \mathrm{H}), 1.03(\mathrm{~s}$, $9 \mathrm{H}), 0.98(\mathrm{~d}, J=6.6 \mathrm{~Hz}, 3 \mathrm{H}) ;{ }^{13} \mathrm{C} \mathrm{NMR}\left(\mathrm{CDCl}_{3}, 75 \mathrm{MHz}\right) \delta 168.5,147.5,135.7,135.7,134.0$, 129.7, 127.7, 126.9, 61.9, 60.5, 39.5, 29.8, 26.9, 19.9, 19.3, 14.4, 12.6 ppm; IR(neat): $v_{\max } 2930$, 1711, $1109 \mathrm{~cm}^{-1}$; HRMS (ESI) $\mathrm{m} / z$ calculated for $\mathrm{C}_{26} \mathrm{H}_{36} \mathrm{O}_{3} \mathrm{SiNa}[\mathrm{M}+\mathrm{Na}]^{+}$447.2331, found 447.2335.

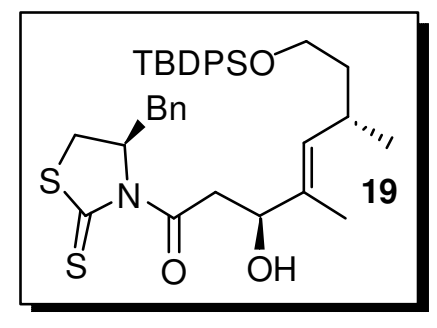

$(3 S, \quad 6 S, \quad E)-1-((R)-4-b e n z y l-2-t h i o x o t h i a z o l i d i n-3-y l)-8-((t e r t-$ butyldiphenylsilyl)oxy)-3-hydroxy-4,6-dimethyloct-4-en-1-one (19): To a solution of ester $\mathbf{1 7}(9.5 \mathrm{~g}, 22.4 \mathrm{mmol})$ in dry $\mathrm{CH}_{2} \mathrm{Cl}_{2}(50$ $\mathrm{mL})$ at $-78{ }^{\circ} \mathrm{C}$ under argon, DIBAL-H $(1.0 \mathrm{M}$ in toluene, $22.4 \mathrm{~mL}$, $22.4 \mathrm{mmol}$ ) was added slowly. The reaction was continued for $15 \mathrm{~min}$ at the same temperature before quenching it with $\mathrm{MeOH}(8 \mathrm{~mL})$. A saturated solution of sodium potassium tartrate (30 $\mathrm{mL}$ ) was added to the reaction mixture. The resulting mixture was warmed to room temperature and stirred vigorous for $2 \mathrm{~h}$ and finally extracted with EtOAc $(2 \times 100 \mathrm{~mL})$, washed with water, brine, dried $\left(\mathrm{Na}_{2} \mathrm{SO}_{4}\right)$ and concentrated in vacuo. Purification of residue by a flash column chromatography produced pure aldehyde as a colorless liquid $\left[\mathrm{R}_{f}=0.58\right.$ (5\% EtOAc in hexane)] which was taken for next reaction without further characterizations.

Freshly distilled $\mathrm{TiCl}_{4}(2.7 \mathrm{~mL}, 24.6 \mathrm{mmol})$ was added drop wise to a stirred solution of thiazolidinethione $18(6.2 \mathrm{~g}, 24.6 \mathrm{mmol})$ dissolved in anhydrous $\mathrm{CH}_{2} \mathrm{Cl}_{2}(40 \mathrm{~mL})$ at $-40{ }^{\circ} \mathrm{C}$ under argon and continued further for $5 \mathrm{~min}$. DIPEA $(4.3 \mathrm{~mL}, 24.6 \mathrm{mmol})$ was added to the reaction mixture in drop wise fashion and stirred for another $10 \mathrm{~min}$. The reaction mixture was then 
cooled to $-78{ }^{\circ} \mathrm{C}$ and the aldehyde from the previous step (dissolved in $20 \mathrm{~mL} \mathrm{CH}_{2} \mathrm{Cl}_{2}$ ) was cannulated into it. The reaction was continued further at $-78{ }^{\circ} \mathrm{C}$ for $45 \mathrm{~min}$ prior to quench with saturated aqueous $\mathrm{NH}_{4} \mathrm{Cl}$ solution $(15 \mathrm{~mL})$. The resulting mixture was warmed to ambient temperature and extracted with EtOAc $(2 \times 100 \mathrm{~mL})$, washed with water, brine, dried $\left(\mathrm{Na}_{2} \mathrm{SO}_{4}\right)$, filtered and concentrated in vacuo. Purification of residue by column chromatography $\left(\mathrm{SiO}_{2}\right.$, 230-400 mesh, 15\% EtOAc in hexane as eluant) resulted compound $\mathbf{1 9}$ as the major aldol product $(10.2 \mathrm{~g}, 72 \%$ over 2 steps, $d r=5: 1)$ as a yellow oil: $\mathrm{R}_{f}=0.31$ (15\% EtOAc in hexane); $[\alpha]_{\mathrm{D}}{ }^{28}=-88.4\left(\right.$ c 3.62, $\left.\mathrm{CHCl}_{3}\right) ;{ }^{1} \mathrm{H}$ NMR $\left(\mathrm{CDCl}_{3}, 300 \mathrm{MHz}\right) \delta$ 7.67-7.64(m, 4H), 7.41-7.27(m, $11 \mathrm{H}), 5.41-5.34(\mathrm{~m}, 1 \mathrm{H}), 5.23(\mathrm{~d}, J=9.6 \mathrm{~Hz}, 1 \mathrm{H}), 4.57-4.53(\mathrm{~m}, 1 \mathrm{H}), 3.61(\mathrm{t}, J=6.3 \mathrm{~Hz}, 2 \mathrm{H})$, $3.43-3.35(\mathrm{~m}, 3 \mathrm{H}), 3.24(\mathrm{dd}, J=12.9,3.9 \mathrm{~Hz}, 1 \mathrm{H}), 3.08-3.01(\mathrm{~m}, 1 \mathrm{H}), 2.88(\mathrm{~d}, J=11.4 \mathrm{~Hz}, 1 \mathrm{H})$, 2.69-2.59(m, 1H), $2.46(\mathrm{bs}, 1 \mathrm{H}), 1.66(\mathrm{~d}, J=0.9 \mathrm{~Hz}, 3 \mathrm{H}), 1.63-1.54(\mathrm{~m}, 1 \mathrm{H}), 1.52-1.39(\mathrm{~m}, 1 \mathrm{H})$, 1.05(s, 9H), 0.94(d, $J=6.9 \mathrm{~Hz}, 3 \mathrm{H}) ;{ }^{13} \mathrm{C} \mathrm{NMR}\left(\mathrm{CDCl}_{3}, 75 \mathrm{MHz}\right) \delta 201.3,173.0,136.5,135.6$, 134.5, 134.2, 134.1, 132.9, 129.6, 129.5, 129.0, 127.7, 127.7, 127.4, 73.2, 68.6, 62.1, 44.6, 40.2, 36.9, 32.2, 28.4, 27.0, 20.8, 19.3, 12.4 ppm; IR(neat): $v_{\max } 3445,3018,1732,1686,1217 \mathrm{~cm}^{-1}$; HRMS (ESI) $m / z$ calculated for $\mathrm{C}_{36} \mathrm{H}_{45} \mathrm{NO}_{3} \mathrm{~S}_{2} \mathrm{SiNa}[\mathrm{M}+\mathrm{Na}]^{+} 654.2508$, found 654.2506.

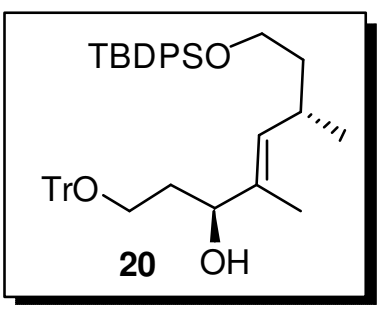

$(3 S, \quad 6 S, \quad E)-8-(($ tert-butyldiphenylsilyl)oxy)-4,6-dimethyl-1(trityloxy)oct-4-en-3-ol (20): To a solution of compound 19 (9.0 g, $14.2 \mathrm{mmol})$ in $\mathrm{MeOH}(30 \mathrm{~mL}), \mathrm{NaBH}_{4}(2.7 \mathrm{~g}, 71.0 \mathrm{mmol})$ was added at $0{ }^{\circ} \mathrm{C}$ and stirred for $30 \mathrm{~min}$ at the same temperature. The reaction was quenched with saturated aqueous $\mathrm{NH}_{4} \mathrm{Cl}(15 \mathrm{~mL})$. $\mathrm{MeOH}$ was removed from the resultant 
mixture under reduced pressure and extracted finally with EtOAc $(3 \times 100 \mathrm{~mL})$, washed with brine, dried $\left(\mathrm{Na}_{2} \mathrm{SO}_{4}\right)$ and concentrated in vacuo. Purification of the residue obtained by column chromatography $\left(\mathrm{SiO}_{2}, 100-200\right.$ mesh, 55\% EtOAc in hexane eluant) afforded the corresponding diol $(5.8 \mathrm{~g}, 92 \%)$ as a thick colorless oil: $\mathrm{R}_{f}=0.41$ in $40 \%$ EtOAc in hexane; $[\alpha]_{\mathrm{D}}{ }^{29}=+1.3(c$ 2.58, $\left.\mathrm{CHCl}_{3}\right) ;{ }^{1} \mathrm{H} \mathrm{NMR}\left(\mathrm{CDCl}_{3}, 300 \mathrm{MHz}\right) \delta$ 7.66-7.64(m, 4H), 7.44-7.34(m, 6H), 5.14(d, $J=$ $9.6 \mathrm{~Hz}, 1 \mathrm{H}), 4.19-4.14(\mathrm{~m}, 1 \mathrm{H}), 3.75-3.71(\mathrm{~m}, 2 \mathrm{H}), 3.59(\mathrm{t}, J=6.6 \mathrm{~Hz}, 2 \mathrm{H}), 2.63-2.61(\mathrm{~m}, 1 \mathrm{H})$, $1.79-1.72(\mathrm{~m}, 1 \mathrm{H}), 1.63-1.55(\mathrm{~m}, 5 \mathrm{H}), 1.59(\mathrm{~s}, 3 \mathrm{H}), 1.46-1.41(\mathrm{~m}, 1 \mathrm{H}), 1.04(\mathrm{~s}, 9 \mathrm{H}), 0.93(\mathrm{~d}, J=6.6$ $\mathrm{Hz}, 3 \mathrm{H}) ;{ }^{13} \mathrm{C} \mathrm{NMR}\left(\mathrm{CDCl}_{3}, 75 \mathrm{MHz}\right) \delta 136.2,135.7,135.7,134.1,132.2,129.7,127.7,77.5$, 62.2, 61.6, 40.2, 36.8, 28.4, 26.9, 20.9, 19.3, 12.0 ppm; IR(neat): $v_{\max } 3367,2930,1427,1109$ $\mathrm{cm}^{-1}$; HRMS (ESI) $\mathrm{m} / z$ calculated for $\mathrm{C}_{26} \mathrm{H}_{38} \mathrm{O}_{3} \mathrm{SiNa}[\mathrm{M}+\mathrm{Na}]^{+} 449.2488$, found 449.2487 .

$\mathrm{Et}_{3} \mathrm{~N}(2.8 \mathrm{~mL}, 19.8 \mathrm{mmol})$ and Trityl chloride $(4.1 \mathrm{~g}, 14.5 \mathrm{mmol})$ were added sequentially to a solution of the above diol $(5.8 \mathrm{~g}, 13.6 \mathrm{mmol})$ in dry $\mathrm{CH}_{2} \mathrm{Cl}_{2}(30 \mathrm{~mL})$ at $0{ }^{\circ} \mathrm{C}$. After being stirred for $15 \mathrm{~min}$ at the same temperature, DMAP (159 $\mathrm{mg}, 1.3 \mathrm{mmol}$ ) was added and the reaction was continued for $6 \mathrm{~h}$ at room temperature. The reaction mixture was quenched with saturated aqueous $\mathrm{NH}_{4} \mathrm{Cl}(15 \mathrm{~mL})$ and extracted with EtOAc $(3 \times 10 \mathrm{~mL})$, washed with brine, dried $\left(\mathrm{Na}_{2} \mathrm{SO}_{4}\right)$, filtered and concentrated in vacuo. Purification of the residue by column chromatography ( $\mathrm{SiO}_{2}, 100-200$ mesh, 20\% EtOAc in hexane eluant) provided pure compound $20(8.1 \mathrm{~g}, 89 \%)$ as a colorless liquid: $\mathrm{R}_{f}=0.45$ in $20 \%$ EtOAc in hexane; $[\alpha]_{\mathrm{D}}{ }^{29}=+12.4(c 1.10$, $\left.\mathrm{CHCl}_{3}\right) ;{ }^{1} \mathrm{H} \mathrm{NMR}\left(\mathrm{CDCl}_{3}, 300 \mathrm{MHz}\right) \delta$ 7.65-7.61(m, 4H), 7.44-7.41(m, 6H), 7.38-7.18(m, 15H), $5.08(\mathrm{~d}, J=9.3 \mathrm{~Hz}, 1 \mathrm{H}), 4.12-4.10(\mathrm{~m}, 1 \mathrm{H}), 3.53(\mathrm{t}, J=6.3 \mathrm{~Hz}, 2 \mathrm{H}), 3.29-3.24(\mathrm{~m}, 1 \mathrm{H}), 3.20-$ 3.15(m, $1 \mathrm{H}), 2.58-2.50(\mathrm{~m}, 2 \mathrm{H}), 1.85-1.82(\mathrm{~m}, 1 \mathrm{H}), 1.68-1.66(\mathrm{~m}, 1 \mathrm{H}), 1.55(\mathrm{~s}, 3 \mathrm{H}), 1.53-1.48(\mathrm{~m}$, 
$1 \mathrm{H}), 1.39-1.34(\mathrm{~m}, 1 \mathrm{H}), 1.02(\mathrm{~s}, 9 \mathrm{H}), 0.87(\mathrm{~d}, J=6.6 \mathrm{~Hz}, 3 \mathrm{H}) ;{ }^{13} \mathrm{C} \mathrm{NMR}\left(\mathrm{CDCl}_{3}, 75 \mathrm{MHz}\right) \delta$ $144.1,135.9,135.7,135.7,134.2,131.9,129.6,128.7,127.9,127.7,127.1,87.3,76.4,62.4,62.2$ 40.2, 35.5, 28.4, 27.0, 20.9, 19.3, 12.0 ppm; IR(neat): $v_{\max } 3433,2930,1109 \mathrm{~cm}^{-1}$; HRMS (ESI) $m / z$ calculated for $\mathrm{C}_{45} \mathrm{H}_{52} \mathrm{O}_{3} \mathrm{SiNa}[\mathrm{M}+\mathrm{Na}]^{+}$691.3583, found 691.3585 .

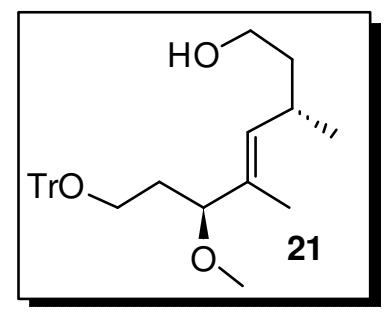

$(3 S, 6 S, E)-6-m e t h o x y-3,5-d i m e t h y l-8-(t r i t y l o x y) o c t-4-e n-1-o l \quad(21)$ : To a chilled $\left(0^{\circ} \mathrm{C}\right)$ solution of alcohol $\mathbf{2 0}(7.8 \mathrm{~g}, 11.7 \mathrm{mmol})$ in dry THF (25 mL) under argon, $\mathrm{NaH}$ (60\% suspension in mineral oil, $560 \mathrm{mg}, 14.0$ mmol) was added and stirred for 30 min prior to quench with MeI (2.2 $\mathrm{mL}, 35.1 \mathrm{mmol})$. The reaction was continued for $6 \mathrm{~h}$ at room temperature before quenching with water at $0{ }^{\circ} \mathrm{C}$. The resultant mixture was extracted with EtOAc $(2 \times 75 \mathrm{~mL})$ washed with brine and dried over $\mathrm{Na}_{2} \mathrm{SO}_{4}$ and concentrated under reduced pressure. The residue was purified by silica gel column chromatography (3\% EtOAc in hexane as eluant) to yield corresponding methyl ether (7.6 g, $95 \%$ yield) as a colorless liquid: $\mathrm{R}_{f}=0.70$ in $10 \%$ EtOAc in hexane; $[\alpha]_{\mathrm{D}}^{29}=+4.2(c$ 2.06, $\left.\mathrm{CHCl}_{3}\right) ;{ }^{1} \mathrm{H} \mathrm{NMR}\left(\mathrm{CDCl}_{3}, 300 \mathrm{MHz}\right) \delta$ 7.66-7.62(m, $\left.4 \mathrm{H}\right), 7.44-7.32(\mathrm{~m}, 12 \mathrm{H}), 7.30-7.16(\mathrm{~m}$, $9 \mathrm{H}), 5.02(\mathrm{~d}, J=9.6 \mathrm{~Hz}, 1 \mathrm{H}), 3.65(\mathrm{dd}, J=8.0,5.4 \mathrm{~Hz}, 1 \mathrm{H}), 3.56-3.47(\mathrm{~m}, 2 \mathrm{H}), 3.15-3.10(\mathrm{~m}, 1 \mathrm{H})$, $3.10(\mathrm{~s}, 3 \mathrm{H}), 3.06-3.00(\mathrm{~m}, 1 \mathrm{H}), 2.63(\mathrm{~m}, 1 \mathrm{H}), 1.86-1.82(\mathrm{~m}, 1 \mathrm{H}), 1.67-1.63(\mathrm{~m}, 1 \mathrm{H}), 1.46(\mathrm{~d}, J=1.2$ $\mathrm{Hz}, 3 \mathrm{H}), 1.35-1.26(\mathrm{~m}, 2 \mathrm{H}), 1.04(\mathrm{~s}, 9 \mathrm{H}), 0.91(\mathrm{~d}, J=6.6 \mathrm{~Hz}, 3 \mathrm{H}) ;{ }^{13} \mathrm{C} \mathrm{NMR}\left(\mathrm{CDCl}_{3}, 75 \mathrm{MHz}\right) \delta$ $144.6,135.7,135.7,134.9,134.2,133.1,129.6,128.8,127.9,127.8,127.7,126.9,86.5,84.2$, 62.1, 60.5, 55.7, 40.1, 34.7, 28.5, 27.0, 21.1, 19.3, 10.8 ppm; IR(neat): $v_{\max } 2930,1448,1111 \mathrm{~cm}^{-}$ ${ }^{1}$; HRMS (ESI) $m / z$ calculated for $\mathrm{C}_{46} \mathrm{H}_{54} \mathrm{O}_{3} \mathrm{SiNa}[\mathrm{M}+\mathrm{Na}]^{+} 705.3740$, found 705.3742 . 
TBAF (1M solution in THF, $12.4 \mathrm{~mL}, 12.4 \mathrm{mmol}$ ) was added to an ice cold solution of the above compound $(6.5 \mathrm{~g}, 9.5 \mathrm{mmol})$ dissolved in dry THF $(25 \mathrm{~mL})$ under argon and stirred further for $3 \mathrm{~h}$ at ambient temperature prior to quench with saturated $\mathrm{NH}_{4} \mathrm{Cl}(10 \mathrm{~mL})$ solution. The resulting mixture was extracted with EtOAc $(3 \times 50 \mathrm{~mL})$, washed with water, brine, dried $\left(\mathrm{Na}_{2} \mathrm{SO}_{4}\right)$, filtered and concentrated in vacuo. Purification by column chromatography $\left(\mathrm{SiO}_{2}\right.$, 100-200 mesh, 15\% EtOAc in hexane as eluant) afforded compound 21 (3.8 g, 90\%) as a colorless oil: $R_{f}=0.43(20 \%$ EtOAc in hexane $) ;[\alpha]_{\mathrm{D}}{ }^{28}=+8.2\left(c 3.36, \mathrm{CHCl}_{3}\right) ;{ }^{1} \mathrm{H} \mathrm{NMR}\left(\mathrm{CDCl}_{3}\right.$, $300 \mathrm{MHz}) \delta 7.45-7.42(\mathrm{~m}, 6 \mathrm{H}), 7.32-7.19(\mathrm{~m}, 9 \mathrm{H}), 5.07(\mathrm{~d}, J=9.3 \mathrm{~Hz}, 1 \mathrm{H}), 3.64(\mathrm{t}, J=6.9,1 \mathrm{H})$, $3.48-3.32(\mathrm{~m}, 2 \mathrm{H}), 3.18-3.10(\mathrm{~m}, 1 \mathrm{H}), 3.13(\mathrm{~s}, 3 \mathrm{H}), 3.06-2.99(\mathrm{~m}, 1 \mathrm{H}), 2.58-2.48(\mathrm{~m}, 1 \mathrm{H}), 1.97-$ 1.86(m, 1H), 1.81-1.69(m, 1H), 1.58-1.47(m, 1H), 1.50(d, $J=1.2 \mathrm{~Hz}, 3 \mathrm{H}), 1.37-1.30(\mathrm{~m}, 1 \mathrm{H})$, $0.96(\mathrm{~d}, J=6.9 \mathrm{~Hz}, 3 \mathrm{H}) ;{ }^{13} \mathrm{C} \mathrm{NMR}\left(\mathrm{CDCl}_{3}, 75 \mathrm{MHz}\right) \delta 144.5,134.8,133.4,128.8,127.8,126.9$, 86.6, 84.3, 61.4, 60.6, 55.7, 40.1, 34.4, 28.9,21.4, 10.7 ppm; IR(neat): $v_{\max } 3412,1645 \mathrm{~cm}^{-1}$; HRMS (ESI) $m / z$ calculated for $\mathrm{C}_{30} \mathrm{H}_{36} \mathrm{O}_{3} \mathrm{Na}[\mathrm{M}+\mathrm{Na}]^{+}$467.2562, found 467.2564.

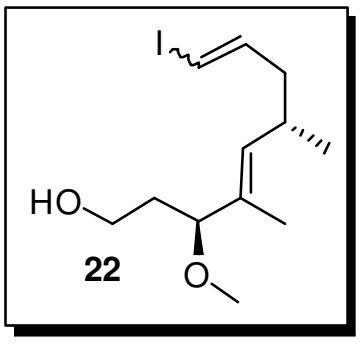

(3S, 4E, 6S, 8E)-9-iodo-3-methoxy-4, 6-dimethylnona-4, 8-dien-1-ol (22): To a stirred solution of alcohol 21 (1.0 g, $2.2 \mathrm{mmol})$ in distilled EtOAc $(15 \mathrm{~mL}), \mathrm{IBX}(1.1 \mathrm{~g}, 4.0 \mathrm{mmol})$ was added and refluxed $\left(80{ }^{\circ} \mathrm{C}\right)$ for $3 \mathrm{~h}$. The reaction mixture was then cooled to room temperature, filtered through a celite pad, washed with EtOAc $(3 \times 15 \mathrm{~mL})$ and concentrated in vacuo. The crude aldehyde $\left(\mathrm{R}_{f}=0.40,10 \%\right.$ EtOAc in hexane) was subjected to flash chromatography and used for the next reaction without further characterizations. 
To a stirred suspension of $\mathrm{CrCl}_{2}(2.2 \mathrm{~g}, 17.6 \mathrm{mmol})$ in dry $\mathrm{THF}(25 \mathrm{~mL})$ at $0{ }^{\circ} \mathrm{C}$ under argon, a THF ( $8 \mathrm{~mL})$ solution of the aldehyde obtained from the previous step and iodoform (2.6 $\mathrm{g}, 6.6 \mathrm{mmol}$ ) was cannulated. The reaction was continued further for $2 \mathrm{~h}$ at the room temperature prior to quench with water. The resultant mixture was extracted with EtOAc $(2 \times 50 \mathrm{~mL})$, washed with brine, dried $\left(\mathrm{Na}_{2} \mathrm{SO}_{4}\right)$, filtered, and concentrated. The crude product was then dissolved in 1:1 $\mathrm{CH}_{2} \mathrm{Cl}_{2} / \mathrm{MeOH}(15 \mathrm{~mL})$, cooled to $0{ }^{\circ} \mathrm{C}$ and CSA $(25 \mathrm{mg}, 0.11 \mathrm{mmol})$ was added. The reaction was quenched with $\mathrm{Et}_{3} \mathrm{~N}(1 \mathrm{~mL})$ after $5 \mathrm{~min}$. The solvent was removed in vacuo and the crude product was purified by column chromatography $\left(\mathrm{SiO}_{2}, 100-200\right.$ mesh, 15\% EtOAc in hexane as eluant) to yield iodo alcohol 22 along with its inseparable mixture of $Z$-isomer (547 mg, $75 \%$ yield, $12 E: 12 Z \sim 4: 1)$ as a colorless liquid: $\mathrm{R}_{f}=0.46$ in $10 \%$ EtOAc in hexane; ${ }^{1} \mathrm{H}$ $\operatorname{NMR}\left(\mathrm{CDCl}_{3}, 500 \mathrm{MHz}\right) \delta 6.42(\mathrm{dt}, J=14.0,7.5 \mathrm{~Hz}, 1 \mathrm{H}), 5.97(\mathrm{~d}, J=14.0 \mathrm{~Hz}, 1 \mathrm{H}), 5.15(\mathrm{~d}, J=$ $10.0 \mathrm{~Hz}, 1 \mathrm{H}), 3.73(\mathrm{~m}, 2 \mathrm{H}), \quad 3.68-3.65(\mathrm{~m}, 1 \mathrm{H}), 3.19(\mathrm{~s}, 3 \mathrm{H}), 2.55-2.51(\mathrm{~m}, 1 \mathrm{H}), \quad 2.44(\mathrm{bs}, 1 \mathrm{H})$, $2.10-2.05(\mathrm{~m}, 1 \mathrm{H}), 1.99-1.89(\mathrm{~m}, 2 \mathrm{H}), 1.66-1.56(\mathrm{~m}, 1 \mathrm{H}), 1.56(\mathrm{~d}, J=1.5 \mathrm{~Hz}, 3 \mathrm{H}), 0.99(\mathrm{~d}, J=6.5$ $\mathrm{Hz}, 3 \mathrm{H}) ;{ }^{13} \mathrm{C} \mathrm{NMR}\left(\mathrm{CDCl}_{3}, 75 \mathrm{MHz}\right) \delta 145.0,133.7,133.6,87.1,75.6,61.5,55.9,43.7,36.5$, 31.9, 20.8, $11.3 \mathrm{ppm}$; IR(neat): $v_{\max } 3491,3020,1217 \mathrm{~cm}^{-1}$; HRMS (ESI) $\mathrm{m} / z$ calculated for $\mathrm{C}_{12} \mathrm{H}_{21} \mathrm{IO}_{2} \mathrm{Na}[\mathrm{M}+\mathrm{Na}]^{+} 347.0484$, found 347.0485

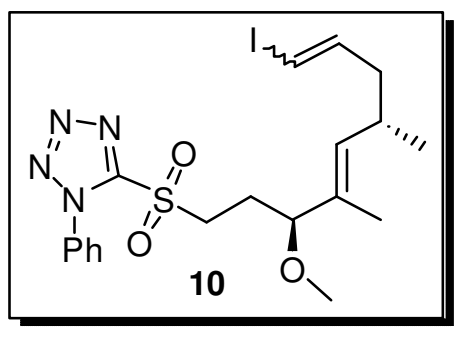

5-(((3S, 4E, 6S, 8E)-9-iodo-3-methoxy-4,6-dimethylnona-4,8dien-1-yl)sulfonyl)-1-phenyl-1H-tetrazole (10): To a mixture of alcohol 22 (220 mg, $0.68 \mathrm{mmol}, 12 E: 12 Z \sim 4: 1), \mathrm{Ph}_{3} \mathrm{P}$ (196 mg, $0.75 \mathrm{mmol}$ ), and 1-phenyl-1H-tetrazol-5-thiol 23 (134 mg, 0.75 
$\mathrm{mmol})$ in dry THF $(5 \mathrm{~mL})$ at $0{ }^{\circ} \mathrm{C}$, DEAD $(0.15 \mathrm{~mL}, 0.75 \mathrm{mmol})$ was added in drop wise manner and stirred for $2 \mathrm{~h}$ at ambient temperature. The reaction mixture was then quenched with saturated aqueous $\mathrm{NaHCO}_{3}(2 \mathrm{~mL})$, extracted with EtOAc $(2 \times 25 \mathrm{~mL})$, washed with brine, dried $\left(\mathrm{Na}_{2} \mathrm{SO}_{4}\right)$ and concentrated under vacuum. The residue was purified by column chromatography $\left(\mathrm{SiO}_{2}, 100-200\right.$ mesh, 5\% EtOAc in hexane as eluant) to afford the corresponding sulfide (306 mg, $93 \%, 12 E: 12 Z \sim 4: 1)$ as a thick oil: $\mathrm{R}_{f}=0.67$ in $10 \%$ EtOAc in hexane; ${ }^{1} \mathrm{H}$ NMR $\left(\mathrm{CDCl}_{3}\right.$, $500 \mathrm{MHz}) \delta 7.60-7.52(\mathrm{~m}, 5 \mathrm{H}), 6.40(\mathrm{dt}, J=14.1,7.2 \mathrm{~Hz}, 1 \mathrm{H}), 5.96(\mathrm{~d}, J=14.1 \mathrm{~Hz}, 1 \mathrm{H}), 5.15(\mathrm{~d}, J$ $=10.0 \mathrm{~Hz}, 1 \mathrm{H}), 3.57-3.54(\mathrm{~m}, 1 \mathrm{H}), 3.44-3.33(\mathrm{~m}, 2 \mathrm{H}), 3.16(\mathrm{~s}, 3 \mathrm{H}), 2.57-2.51(\mathrm{~m}, 1 \mathrm{H}), 2.11-$ $2.04(\mathrm{~m}, 2 \mathrm{H}), 2.01-1.93(\mathrm{~m}, 2 \mathrm{H}), 1.54(\mathrm{~s}, 3 \mathrm{H}), 0.99(\mathrm{~d}, J=6.5 \mathrm{~Hz}, 3 \mathrm{H}) ;{ }^{13} \mathrm{C} \mathrm{NMR}\left(\mathrm{CDCl}_{3}, 75\right.$ MHz) $\delta 154.5,144.9,134.5,133.9,133.0,130.2,129.9,124.1,85.6,75.8,55.9,43.6,33.5,31.9$, 30.2, 20.8, $11.1 \mathrm{ppm}$; IR(neat): $v_{\max }$ 2857, $1093 \mathrm{~cm}^{-1}$; HRMS (ESI) $\mathrm{m} / \mathrm{z}$ calculated for $\mathrm{C}_{19} \mathrm{H}_{25} \mathrm{IN}_{4} \mathrm{OSNa}[\mathrm{M}+\mathrm{Na}]^{+}$507.0691, found 507.0694

To an ice cold ethanolic solution $(5 \mathrm{~mL})$ of the above sulfide $(275 \mathrm{mg}, 0.57 \mathrm{mmol}$, $12 E: 12 \mathrm{Z} \sim 4: 1),\left(\mathrm{NH}_{4}\right)_{6} \mathrm{Mo}_{7} \mathrm{O}_{24} \cdot 4 \mathrm{H}_{2} \mathrm{O}(35 \mathrm{mg}, 0.03 \mathrm{mmol})$ and $30 \%(\mathrm{w} / \mathrm{w})$ aqueous $\mathrm{H}_{2} \mathrm{O}_{2}$ solution $(1 \mathrm{~mL})$ were added sequentially. The reaction was stirred for $12 \mathrm{~h}$ at room temperature. The reaction mixture was then diluted with saturated aqueous $\mathrm{NaHCO}_{3}$ solution $(2 \mathrm{~mL})$ and extracted with EtOAc $(2 \times 25 \mathrm{~mL})$, washed with brine, dried over $\mathrm{Na}_{2} \mathrm{SO}_{4}$, filtered, and concentrated. The crude material was purified by column chromatography $\left(\mathrm{SiO}_{2}, 100-200\right.$ mesh, $5 \%$ EtOAc in hexane as eluant) to get sulfone $10(240 \mathrm{mg}, 12 E: 12 Z \sim 4: 1,82 \%)$ as a pale yellow oil: $R_{f}=0.60\left(10 \%\right.$ EtOAc in hexane); ${ }^{1} \mathrm{H} \mathrm{NMR}\left(\mathrm{CDCl}_{3}, 400 \mathrm{MHz}\right) \delta 7.70-7.67(\mathrm{~m}, 2 \mathrm{H}), 7.62-$ 7.57(m, 3H), 6.4(dt, $J=14.4,7.2 \mathrm{~Hz}, 1 \mathrm{H}), 5.69(\mathrm{~d}, J=14.4 \mathrm{~Hz}, 1 \mathrm{H}), 5.19(\mathrm{~d}, J=9.6 \mathrm{~Hz}, 1 \mathrm{H})$, 
3.82-3.74(m, $2 \mathrm{H}), 3.60-3.57(\mathrm{~m}, 1 \mathrm{H}), 3.16(\mathrm{~s}, 3 \mathrm{H}), 2.53(\mathrm{~m}, 1 \mathrm{H}), 2.21-2.17(\mathrm{~m}, 1 \mathrm{H}), 2.11-2.04(\mathrm{~m}$, 2H), 2.00-1.95(m, $1 \mathrm{H}), 1.54(\mathrm{~d}, J=0.8 \mathrm{~Hz}, 3 \mathrm{H}), 0.98(\mathrm{~d}, J=6.4 \mathrm{~Hz}, 3 \mathrm{H}) ;{ }^{13} \mathrm{C} \mathrm{NMR}\left(\mathrm{CDCl}_{3}, 75\right.$ MHz) $\delta 153.6,144.8,134.8,133.1,132.3,131.5,129.8,125.2,84.3,75.8,56.0,53.2,43.5,31.9$, 26.7, 20.6, 11.2 ppm; IR(neat): $v_{\max } 2924,1342 \mathrm{~cm}^{-1}$; HRMS (ESI) $\mathrm{m} / \mathrm{z}$ calculated for $\mathrm{C}_{19} \mathrm{H}_{25} \mathrm{IN}_{4} \mathrm{O}_{3} \mathrm{SNa}[\mathrm{M}+\mathrm{Na}]^{+} 539.0590$, found 539.0593 .

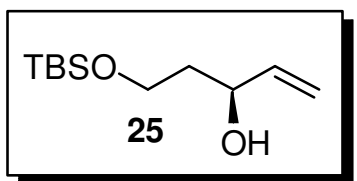

(S)-5-((tert-butyldimethylsilyl)oxy)pent-1-en-3-ol (25): ${ }^{\mathrm{n}} \mathrm{BuLi}(1.6 \mathrm{M}$ in hexane, $3.9 \mathrm{~mL}, 6.3 \mathrm{mmol})$ was added to a suspension of $\mathrm{Me}_{3} \mathrm{SI}(1.5$ g, $7.4 \mathrm{mmol})$ in anhydrous THF $(20 \mathrm{~mL})$ at $-40{ }^{\circ} \mathrm{C}$ under argon. After $30 \mathrm{~min}$ of stirring, epoxide $24(500 \mathrm{mg}, 2.5 \mathrm{mmol})$ dissolved in THF $(10 \mathrm{~mL})$ was cannulated. The reaction was warmed to $0{ }^{\circ} \mathrm{C}$ within $30 \mathrm{~min}$ and stirred further for $2 \mathrm{~h}$ at the same temperature. The reaction was quenched finally with water, extracted with EtOAc $(3 \times 25 \mathrm{~mL})$ washed with brine, dried over $\mathrm{Na}_{2} \mathrm{SO}_{4}$, filtered, and concentrated in vacuo. The residue was purified by column chromatography $\left(\mathrm{SiO}_{2}, 100-200\right.$ mesh, $8 \%$ EtOAc in hexane as eluant) to obtain allylic alcohol 25 (481 mg, 90\%) as a pale yellow oil: $R_{f}=0.28\left(10 \%\right.$ EtOAc in hexane); $[\alpha]_{\mathrm{D}}^{29}=+3.3(c 3.24$, $\left.\mathrm{CHCl}_{3}\right) ;{ }^{1} \mathrm{H} \mathrm{NMR}\left(\mathrm{CDCl}_{3}, 300 \mathrm{MHz}\right) \delta 5.92-5.81(\mathrm{~m}, 1 \mathrm{H}), 5.26(\mathrm{~d}, J=17.1 \mathrm{~Hz}, 1 \mathrm{H}), 5.09(\mathrm{~d}, J=$ $10.5 \mathrm{~Hz}, 1 \mathrm{H}), 4.34(\mathrm{bs}, 1 \mathrm{H}), 3.91-3.75(\mathrm{~m}, 2 \mathrm{H}), 3.35-3.34(\mathrm{~m}, 1 \mathrm{H}), 1.78-1.68(\mathrm{~m}, 2 \mathrm{H}), 0.89(\mathrm{~s}, 9 \mathrm{H})$, 0.06(s, 6H); ${ }^{13} \mathrm{C} \mathrm{NMR}\left(\mathrm{CDCl}_{3}, 75 \mathrm{MHz}\right) \delta 140.7,114.2,72.5,61.9,38.3,25.9,18.2,-5.5,-5.5$ ppm; IR(neat): $v_{\max } 3422,2927,1097 \mathrm{~cm}^{-1}$; HRMS (ESI) $\mathrm{m} / z$ calculated for $\mathrm{C}_{11} \mathrm{H}_{24} \mathrm{O}_{2} \mathrm{SiNa}$ $[\mathrm{M}+\mathrm{Na}]^{+} 239.1443$, found 239.1445. 


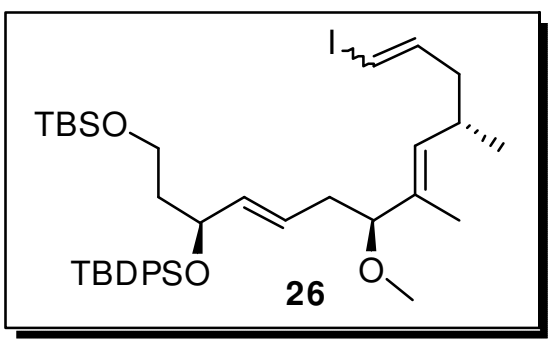

$(S)-5-((1 E, \quad 4 S, \quad 5 E, \quad 7 S, \quad 9 E)-10$-iodo-4-methoxy-5,7dimethyldeca-1,5,9-trien-1-yl)-2,2,9,9,10,10-hexamethyl3,3-diphenyl-4,8-dioxa-3,9-disilaundecane (26): To an ice cold solution of allylic alcohol $25(400 \mathrm{mg}, 1.85 \mathrm{mmol})$ in anhydrous $\mathrm{CH}_{2} \mathrm{Cl}_{2}(10 \mathrm{~mL})$, imidazole (189 mg, $\left.2.78 \mathrm{mmol}\right)$ and TBDPSCl (0.6 mL, $\left.2.22 \mathrm{mmol}\right)$ were added sequentially. The reaction mixture was stirred at room temperature for $5 \mathrm{~h}$ and then quenched by saturated aqueous $\mathrm{NH}_{4} \mathrm{Cl}$ solution $(5 \mathrm{~mL})$. The resultant mixture was extracted with EtOAc $(2 \times 15 \mathrm{~mL})$, washed brine, dried $\left(\mathrm{Na}_{2} \mathrm{SO}_{4}\right)$, filtered and concentrated in vacuo. Purification of the reside by column chromatography $\left(\mathrm{SiO}_{2}, 100-200\right.$ mesh, $2 \%$ EtOAc in hexane as eluant) furnished corresponding TBDPS protected olefin ( $841 \mathrm{mg}$, quantitative) as a colorless thick oil: $R_{f}=0.80\left(10 \%\right.$ EtOAc in hexane); $[\alpha]_{\mathrm{D}}{ }^{28}=+4.99\left(c 3.70, \mathrm{CHCl}_{3}\right) ;{ }^{1} \mathrm{H} \mathrm{NMR}\left(\mathrm{CDCl}_{3}\right.$, $300 \mathrm{MHz}) \delta$ 7.69-7.63(m, 4H), 7.41-7.32(m, 6H), 5.84-5.73(m, 1H), 4.95-4.89(m, 2H), 4.344.28(m, 1H), 3.61-3.53(m, 2H), 1.86-1.76(m, 1H), 1.70-1.59(m, 1H), 1.06(s, 9H), 0.82(s, 9H), 0.04(s, 6H); ${ }^{13} \mathrm{C} \mathrm{NMR}\left(\mathrm{CDCl}_{3}, 75 \mathrm{MHz}\right) \delta 140.7,136.1,136.0,134.4,129.6,129.5,127.6$, 127.4, 114.5, 72.4, 59.6, 40.9, 27.2, 26.0, 19.5, 18.3, -5.2 ppm; IR(neat): $v_{\max } 2930,1108 \mathrm{~cm}^{-1}$; HRMS (ESI) $m / z$ calculated for $\mathrm{C}_{27} \mathrm{H}_{42} \mathrm{O}_{2} \mathrm{Si}_{2} \mathrm{Na}[\mathrm{M}+\mathrm{Na}]^{+} 477.2621$, found 477.2622.

To an ice cold stirred solution of above olefin $(264 \mathrm{mg}, 0.58 \mathrm{mmol})$ in $\mathrm{THF} / \mathrm{B} \mathrm{BuOH}(2: 1$, $\mathrm{v} / \mathrm{v}, 4 \mathrm{~mL})$, NMO (136 mg, $1.16 \mathrm{mmol})$ and $\mathrm{OsO}_{4}\left(0.2 \mathrm{M}\right.$ solution in $\left.{ }^{\mathrm{t}} \mathrm{BuOH}, 0.3 \mathrm{~mL}\right)$ were added sequentially. The resultant mixture was further stirred for $12 \mathrm{~h}$ at room temperature. The solvent was then removed in vacuo and the crude mixture was filtered through a short pad of silica. The filtrate was collected, concentrated and dissolved in 4:1 THF/ $\mathrm{H}_{2} \mathrm{O}(6 \mathrm{~mL})$. The mixture was 
cooled to $0{ }^{\circ} \mathrm{C}$ and $\mathrm{NaIO}_{4}(620 \mathrm{mg}, 2.9 \mathrm{mmol})$ and $\mathrm{NaHCO}_{3}(292 \mathrm{mg}, 3.5 \mathrm{mmol})$ were added sequentially. The reaction mixture was warmed to room temperature and stirred further for 30 min. The reaction mixture was diluted with EtOAc $(10 \mathrm{~mL})$, filtered through a short pad of celite and the filtrate was concentrated to yield crude aldehyde $\mathbf{1 1}$ which was directly taken in JuliaKocienski olefination without further purification and characterizations.

To a stirred solution of sulfone 10 (330 mg, $0.64 \mathrm{mmol}, 12 E: 12 Z \sim 4: 1)$ in dry THF (5 $\mathrm{mL})$ at $-78{ }^{\circ} \mathrm{C}$ under argon, KHMDS $(1.3 \mathrm{~mL}, 0.64 \mathrm{mmol}, 0.5 \mathrm{M}$ in toluene) was added and the reaction mixture was stirred for $30 \mathrm{~min}$ at the same temperature. A solution of the above aldehyde $\mathbf{1 1}$ dissolved in dry THF ( $2 \mathrm{~mL}$ ) was cannulated to the reaction mixture and stirred for another $2 \mathrm{~h}$ at the same temperature before quenching it with saturated aqueous $\mathrm{NH}_{4} \mathrm{Cl}$ solution $(2 \mathrm{~mL})$. The resultant mixture was extracted with EtOAc $(2 \times 10 \mathrm{~mL})$, washed with brine, dried $\left(\mathrm{Na}_{2} \mathrm{SO}_{4}\right)$, filtered and concentrated in vacuo. Purification by column chromatography $\left(\mathrm{SiO}_{2}\right.$, 230-400 mesh, 2\% EtOAc in hexane as eluant) furnished corresponding $4 E$-olefin 26 (339 mg, $12 E: 12 Z \sim 4: 1,71 \%)$ as a colorless liquid: $R_{f}=0.48\left(5 \%\right.$ EtOAc in hexane); ${ }^{1} \mathrm{H} \mathrm{NMR}\left(\mathrm{CDCl}_{3}\right.$, $300 \mathrm{MHz}) \delta 7.68-7.62(\mathrm{~m}, 4 \mathrm{H}), 7.40-7.30(\mathrm{~m}, 6 \mathrm{H}), 6.36(\mathrm{dt}, J=14.4,7.2 \mathrm{~Hz}, 1 \mathrm{H}), 5.88(\mathrm{~d}, J=14.4$ $\mathrm{Hz}, 1 \mathrm{H}), 5.44(\mathrm{dd}, J=15.4,7.2 \mathrm{~Hz}, 1 \mathrm{H}), 5.24-5.16(\mathrm{~m}, 1 \mathrm{H}), 4.97(\mathrm{~d}, J=8.7 \mathrm{~Hz}, 1 \mathrm{H}), 4.29-4.24(\mathrm{~m}$, $1 \mathrm{H}), 3.59(\mathrm{t}, J=6.9 \mathrm{~Hz}, 2 \mathrm{H}), 3.22(\mathrm{t}, J=6.9 \mathrm{~Hz}, 1 \mathrm{H}), 3.09(\mathrm{~s}, 3 \mathrm{H}), 2.51-2.41(\mathrm{~m}, 1 \mathrm{H}), 2.17-2.10(\mathrm{~m}$, $1 \mathrm{H}), 2.04-1.99(\mathrm{~m}, 1 \mathrm{H}), 1.97-1.89(\mathrm{~m}, 2 \mathrm{H}), 1.87-1.77(\mathrm{~m}, 1 \mathrm{H}), 1.67-1.63(\mathrm{~m}, 1 \mathrm{H}), 1.46(\mathrm{~d}, J=1.2$ $\mathrm{Hz}, 3 \mathrm{H}), 1.03(\mathrm{~s}, 9 \mathrm{H}), 0.94(\mathrm{~d}, J=6.6 \mathrm{~Hz}, 3 \mathrm{H}), 0.83(\mathrm{~s}, 9 \mathrm{H}),-0.03(\mathrm{~s}, 6 \mathrm{H}) ;{ }^{13} \mathrm{C} \mathrm{NMR}\left(\mathrm{CDCl}_{3}, 75\right.$ MHz) $\delta 145.0,136.1,136.0,134.7,134.6,133.8,133.4,129.6,129.4,127.6,127.4,127.2,87.1$, $75.5,72.1,59.8,55.7,43.5,41.4,36.8,31.8,27.2,26.1,20.6,19.5,18.4,10.9,-5.2 \mathrm{ppm}$ IR(neat): $v_{\max } 2928,1107 \mathrm{~cm}^{-1}$; HRMS (ESI) $m / z$ calculated for $\mathrm{C}_{38} \mathrm{H}_{59} \mathrm{IO}_{3} \mathrm{Si}_{2} \mathrm{Na}[\mathrm{M}+\mathrm{Na}]^{+}$ 


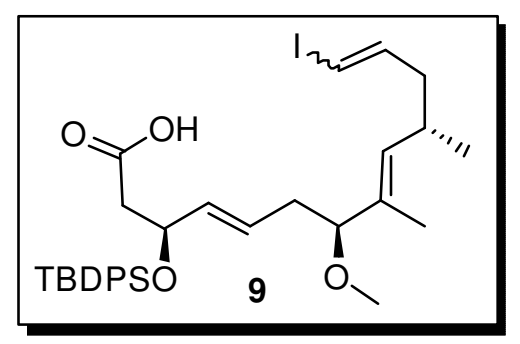

$(3 S, 4 E, 7 S, 8 E, 10 S, 12 E)-3-((t e r t-b u t y l d i p h e n y l s i l y l) o x y)-13-$ iodo-7-methoxy-8,10-dimethyltrideca-4,8,12-trienoic acid (9): To an ice cold solution of olefin 26 (272 $\mathrm{mg}, 0.36 \mathrm{mmol}$, $12 E: 12 Z \sim 4: 1)$ in anhydrous $\mathrm{CH}_{2} \mathrm{Cl}_{2} / \mathrm{MeOH}(4: 1,8 \mathrm{~mL})$ under argon, CSA ( $9 \mathrm{mg}, 0.04 \mathrm{mmol}$ ) was added. The reaction was continued for $6 \mathrm{~h}$ at $0{ }^{\circ} \mathrm{C}$ before quenching it with $\mathrm{Et}_{3} \mathrm{~N}(0.2 \mathrm{~mL})$. The resultant mixture was concentrated and purified by column chromatography $\left(\mathrm{SiO}_{2}, 100-200\right.$ mesh, $15 \%$ EtOAc in hexane as eluant) to provide the required TBS deprotected alcohol (180 mg, 12E:12Z 4:1, 78\%) as a colorless oil: $\mathrm{R}_{f}=0.30$ (10\% EtOAc in hexane); ${ }^{1} \mathrm{H}$ NMR $\left(\mathrm{CDCl}_{3}, 300 \mathrm{MHz}\right) \delta$ 7.69-7.64(m, 4H), 7.43-7.25(m, 6H), 6.37(dt, $J=$ 14.7, $6.6 \mathrm{~Hz}, 1 \mathrm{H}), 5.89(\mathrm{~d}, J=14.7 \mathrm{~Hz}, 1 \mathrm{H}), 5.53(\mathrm{dd}, J=15.3,6.0 \mathrm{~Hz}, 1 \mathrm{H}), 5.37-5.27(\mathrm{~m}, 1 \mathrm{H})$, $5.00(\mathrm{~d}, J=9.0 \mathrm{~Hz}, 1 \mathrm{H}), 4.41-4.32(\mathrm{~m}, 1 \mathrm{H}), 3.69(\mathrm{bs}, 2 \mathrm{H}), 3.28(\mathrm{t}, J=6.6 \mathrm{~Hz}, 1 \mathrm{H}), 3.10(\mathrm{~s}, 3 \mathrm{H})$, 2.52-2.42(m, $1 \mathrm{H}), 2.22-2.13(\mathrm{~m}, 2 \mathrm{H}), 2.09-2.01(\mathrm{~m}, 1 \mathrm{H}), 1.98-1.84(\mathrm{~m}, 2 \mathrm{H}), 1.80-1.72(\mathrm{~m}, 1 \mathrm{H})$ $1.47(\mathrm{~d}, J=1.2 \mathrm{~Hz}, 3 \mathrm{H}), 1.06(\mathrm{~s}, 9 \mathrm{H}), 0.94(\mathrm{~d}, J=6.9 \mathrm{~Hz}, 3 \mathrm{H}) ;{ }^{13} \mathrm{C} \mathrm{NMR}\left(\mathrm{CDCl}_{3}, 75 \mathrm{MHz}\right) \delta$ 145.0, 136.2, 136.0, 134.4, 134.3, 134.1, 134.1, 133.9, 133.4, 129.9, 129.7, 127.8, 127.6, 127.4, 86.7, 75.6, 73.2, 59.6, 55.7, 43.5, 40.1, 36.9, 31.9, 27.2, 20.7, 19.4, 11.0 ppm; IR(neat): $v_{\max }$ 3445, 2930, $1107 \mathrm{~cm}^{-1}$; HRMS (ESI) $\mathrm{m} / z$ calculated for $\mathrm{C}_{32} \mathrm{H}_{45} \mathrm{IO}_{3} \mathrm{SiNa}[\mathrm{M}+\mathrm{Na}]^{+}$655.2080, found 655.2083.

The above alcohol (176 g, $0.28 \mathrm{mmol}, 12 E: 12 Z \sim 4: 1)$ was first treated with IBX (156 
$\mathrm{mg}, 0.56 \mathrm{mmol}$ ), according to the procedure described in the oxidation of alcohol 21 , to yield the corresponding aldehyde $\left[\mathrm{R}_{f}=0.60\right.$ (8\% EtOAc in hexane) $]$ which was taken forward without further purification and characterizations.

To a stirred solution of the above aldehyde in ${ }^{\mathrm{t}} \mathrm{BuOH} / 2$-methyl-2-butene $(2: 1,6 \mathrm{~mL})$ at room temperature, a freshly prepared mixture of aqueous solution of $\mathrm{NaClO}_{2}(76 \mathrm{mg}, 0.84 \mathrm{mmol})$ and $\mathrm{NaH}_{2} \mathrm{PO}_{4} \cdot 2 \mathrm{H}_{2} \mathrm{O}(131 \mathrm{mg}, 0.84 \mathrm{mmol})$ was added. The reaction was continued for $3 \mathrm{~h}$ at room temperature prior to extract with EtOAc $(3 \times 5 \mathrm{~mL})$. The organic layer was washed with brine, dried $\left(\mathrm{Na}_{2} \mathrm{SO}_{4}\right)$, filtered, and concentrated in vacuo. Purification of the crude residue by column chromatography $\left(\mathrm{SiO}_{2}, 60-120\right.$ mesh, $25 \%$ EtOAc in hexane as eluant) provided carboxylic acid 9 (142 mg, 12E:12Z 4:1, 79\% over two steps) as a colorless oil: $\mathrm{R}_{f}=0.22$ (15\% EtOAc in hexane); ${ }^{1} \mathrm{H}$ NMR $\left(\mathrm{CDCl}_{3}, 300 \mathrm{MHz}\right) \delta 7.65-7.63(\mathrm{~m}, 4 \mathrm{H}), 7.41-7.35(\mathrm{~m}, 6 \mathrm{H})$, $6.35(\mathrm{dt}, J=14.1,7.5 \mathrm{~Hz}, 1 \mathrm{H}), 5.88(\mathrm{~d}, J=14.1 \mathrm{~Hz}, 1 \mathrm{H}), 5.50(\mathrm{dd}, J=15.3,7.2 \mathrm{~Hz}, 1 \mathrm{H}), 5.33-$ 5.24(m, 1H), 4.69(dd, $J=9.6,0.6 \mathrm{~Hz}, 1 \mathrm{H}), 4.58-4.52(\mathrm{~m}, 1 \mathrm{H}), 3.20(\mathrm{t}, J=6.9 \mathrm{~Hz}, 1 \mathrm{H}), 3.07(\mathrm{~s}$, $3 \mathrm{H}), 2.53-2.40(\mathrm{~m}, 3 \mathrm{H}), 2.17-2.15(\mathrm{~m}, 1 \mathrm{H}), 1.96-1.82(\mathrm{~m}, 3 \mathrm{H}), 1.44(\mathrm{~d}, J=1.2 \mathrm{~Hz}, 3 \mathrm{H}), 1.02(\mathrm{~s}$, $9 \mathrm{H}), 0.93(\mathrm{~d}, J=6.6 \mathrm{~Hz}, 3 \mathrm{H}) ;{ }^{13} \mathrm{C} \mathrm{NMR}\left(\mathrm{CDCl}_{3}, 75 \mathrm{MHz}\right) \delta 174.8,145.0,136.2,136.0,133.9$, 133.6, 133.5, 133.4, 132.5, 129.9, 129.8, 129.1, 127.8, 127.6, 86.7, 75.6, 71.4, 55.7, 43.5, 43.1, 36.8, 31.9, 27.1, 20.7, 19.4, 11.0 ppm; IR(neat): $v_{\max } 3451,2930,1713 \mathrm{~cm}^{-1}$; HRMS (ESI) $\mathrm{m} / \mathrm{z}$ calculated for $\mathrm{C}_{32} \mathrm{H}_{43} \mathrm{IO}_{4} \mathrm{SiNa}[\mathrm{M}+\mathrm{Na}]^{+}$669.1873, found 669.1874 . 


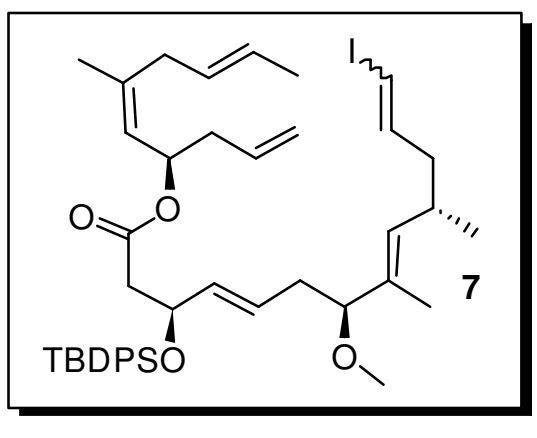

$(R, 5 Z, 8 E)$-6-methyldeca-1, 5, 8-trien-4-yl $(3 S, 4 E, 7 S, 8 E$, $10 S, \quad 12 E)-3-(($ tert-butyldiphenylsilyl) oxy)-13-iodo-7methoxy-8, 10-dimethyltrideca-4, 8, 12-trienoate (7): To a stirred solution of carboxylic acid $9(83 \mathrm{mg}, 0.13 \mathrm{mmol}$, $12 E: 12 Z \sim 4: 1)$ and alcohol 8 (together with ent-8, $28 \mathrm{mg}$, 0.17) in anhydrous $\mathrm{CH}_{2} \mathrm{Cl}_{2}(5 \mathrm{~mL})$ at room temperature under argon atmosphere, DMAP $(22 \mathrm{mg}$, $0.18 \mathrm{mmol})$, MNBA $(72 \mathrm{mg}, 0.21 \mathrm{mmol})$ and $\mathrm{Et}_{3} \mathrm{~N}(0.1 \mathrm{~mL}, 0.65 \mathrm{mmol})$ were added sequentially. Stirring was continued at room temperature for $2 \mathrm{~h}$. The reaction mixture was then diluted with water and extracted with EtOAc $(2 \times 15 \mathrm{~mL})$, washed with brine, dried over $\mathrm{Na}_{2} \mathrm{SO}_{4}$, filtered, and concentrated in vacuo. The residue was purified by column chromatography $\left(\mathrm{SiO}_{2}\right.$, 230-400 mesh, 2\% EtOAc in hexane as eluant) to get ester 7 (74 $\mathrm{mg}, 12 E / 12 Z \sim 4: 1,73 \%)$ as a colorless oil: $\mathrm{R}_{f}=0.72\left(5 \%\right.$ EtOAc in hexane); ${ }^{1} \mathrm{H}$ NMR $\left(\mathrm{CDCl}_{3}, 300 \mathrm{MHz}\right) \delta$ 7.68-7.64(m, 4H), 7.41-7.32(m, 6H), 6.42-6.32(m, $1 \mathrm{H}), 5.91(\mathrm{~d}, J=14.4 \mathrm{~Hz}, 1 \mathrm{H}), 5.70-5.58(\mathrm{~m}, 1 \mathrm{H}), 5.53-5.40(\mathrm{~m}$, $3 \mathrm{H}), 5.35-5.20(\mathrm{~m}, 2 \mathrm{H}), 5.11-4.95(\mathrm{~m}, 4 \mathrm{H}), 4.61-4.54(\mathrm{~m}, 1 \mathrm{H}), 3.19(\mathrm{t}, J=6.6 \mathrm{~Hz}, 1 \mathrm{H}), 3.08(\mathrm{~s}, 3 \mathrm{H})$, $2.89(\mathrm{dd}, J=14.4,6.6 \mathrm{~Hz}, 1 \mathrm{H}), 2.64(\mathrm{dd}, J=14.4,6.6 \mathrm{~Hz}, 1 \mathrm{H}), 2.57-2.37(\mathrm{~m}, 3 \mathrm{H}), 2.30-2.22(\mathrm{~m}$, $2 \mathrm{H}), 2.14-2.06(\mathrm{~m}, 1 \mathrm{H}), 2.00-1.88(\mathrm{~m}, 3 \mathrm{H}), 1.66(\mathrm{~d}, J=1.5 \mathrm{~Hz}, 3 \mathrm{H}), 1.63(\mathrm{dd}, J=6.2,1.5 \mathrm{~Hz}, 3 \mathrm{H})$, $1.45(\mathrm{~d}, J=1.5 \mathrm{~Hz}, 3 \mathrm{H}), 1.01(\mathrm{~s}, 9 \mathrm{H}), 0.94(\mathrm{~d}, J=6.6 \mathrm{~Hz}, 3 \mathrm{H}) ;{ }^{13} \mathrm{C} \mathrm{NMR}\left(\mathrm{CDCl}_{3}, 75 \mathrm{MHz}\right) \delta$ 170.0, 145.0, 142.6, 139.6, 136.2, 136.0, 134.4, 134.2, 133.8, 133.7, 133.4, 133.4, 129.7, 129.6, $128.2,127.6,127.5,126.6,123.8,117.7,86.8,75.6,71.6,70.4,55.7,44.1,43.5,39.7,36.8,35.9$, 31.8, 27.1, 23.5, 20.6, 19.4, 17.9, 11.0 ppm; IR(neat): $v_{\max } 2930,1735,1106 \mathrm{~cm}^{-1}$; HRMS (ESI) $m / z$ calculated for $\mathrm{C}_{43} \mathrm{H}_{59} \mathrm{IO}_{4} \mathrm{SiNa}[\mathrm{M}+\mathrm{Na}]^{+}$817.3125, found 817.3127. 


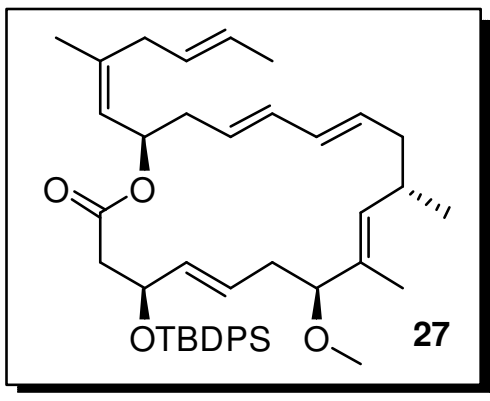

$(4 S, \quad 5 E, \quad 8 S, \quad 9 E, \quad 11 S, \quad 13 E, \quad 15 E, \quad 18 R)-4-((t e r t-$ butyldiphenylsilyl)oxy)-8-methoxy-9,11-dimethyl-18-((1Z, 4E)-2-methylhexa-1,4-dien-1-yl)oxacyclooctadeca-5,9,13,15tetraen-2-one (27): To a solution of compound 7 (70 $\mathrm{mg}, 0.09$ mmol, 12E:12Z 4:1) in anhydrous and degassed DMF (25 mL), $\mathrm{Pd}(\mathrm{OAc})_{2}(20 \mathrm{mg}, 0.09 \mathrm{mmol}), \mathrm{Bu}_{4} \mathrm{NCl}(100 \mathrm{mg}, 0.36 \mathrm{mmol})$ and $\mathrm{K}_{2} \mathrm{CO}_{3}(124 \mathrm{mg}, 0.9 \mathrm{mmol})$ were added. The reaction mixture was stirred for $1 \mathrm{~h}$ at $60{ }^{\circ} \mathrm{C}$. The mixture was cooled to room temperature and filtered through a celite pad. The filtrate was concentrated under vacuum and purified by column chromatography $\left(\mathrm{SiO}_{2}, 230-400\right.$ mesh, 5\% EtOAc in hexane as eluant) to give corresponding macrocycle $27(34 \mathrm{mg}, 58 \%)$ as a pale yellow oil: $\mathrm{R}_{f}=0.65$ (5\% EtOAc in hexane); $[\alpha]_{\mathrm{D}}{ }^{29}=-24.2\left(c 0.33, \mathrm{CHCl}_{3}\right) ;{ }^{1} \mathrm{H} \mathrm{NMR}\left(\mathrm{CDCl}_{3}, 300 \mathrm{MHz}\right) \delta$ 7.69-7.66(m, 4H), 7.417.34(m, 6H), 5.87-5.69(m, 2H), 5.48-5.25(m, 6H), 5.14-5.03(m, 2H), 4.85(d, $J=9.6 \mathrm{~Hz}, 1 \mathrm{H})$ $4.54-4.48(\mathrm{~m}, 1 \mathrm{H}), 3.17-3.12(\mathrm{~m}, 1 \mathrm{H}), 3.10(\mathrm{~s}, 3 \mathrm{H}), 2.92(\mathrm{dd}, J=14.8,6.0 \mathrm{~Hz}, 1 \mathrm{H}), 2.65(\mathrm{dd}, J=$ 14.8, $6.0 \mathrm{~Hz}, 1 \mathrm{H}), 2.57-2.50(\mathrm{~m}, 2 \mathrm{H}), 2.31(\mathrm{dd}, J=14.4,6.0 \mathrm{~Hz}, 1 \mathrm{H}), 2.24-2.15(\mathrm{~m}, 3 \mathrm{H}), 2.10-$ $2.05(\mathrm{~m}, 2 \mathrm{H}), 1.82-1.74(\mathrm{~m}, 1 \mathrm{H}), 1.65-1.63(\mathrm{~m}, 6 \mathrm{H}), 1.44(\mathrm{~d}, J=1.2 \mathrm{~Hz}, 3 \mathrm{H}), 1.03(\mathrm{~s}, 9 \mathrm{H}), 0.98(\mathrm{~d}, J$ $=6.6 \mathrm{~Hz}, 3 \mathrm{H}) ;{ }^{13} \mathrm{C} \mathrm{NMR}\left(\mathrm{CDCl}_{3}, 125 \mathrm{MHz}\right) \delta 170.1,138.7,136.5,136.2,136.1,134.5,134.5$, $133.9,133.8,132.3,131.9,130.8,129.7,129.6,128.3,127.9,127.7,127.6,126.6,126.5,123.9$, 87.6, 72.4, 70.5, 55.4, 44.8, 40.4, 38.3, 36.3, 35.9, 32.7, 27.2, 23.6, 22.2, 19.5, 17.9, $10.1 \mathrm{ppm}$; IR(neat): $v_{\max }$ 2924, $1736 \mathrm{~cm}^{-1}$; HRMS (ESI) $\mathrm{m} / z$ calculated for $\mathrm{C}_{43} \mathrm{H}_{58} \mathrm{O}_{4} \mathrm{SiNa}[\mathrm{M}+\mathrm{Na}]^{+}$ 689.4002, found 689.4005. 


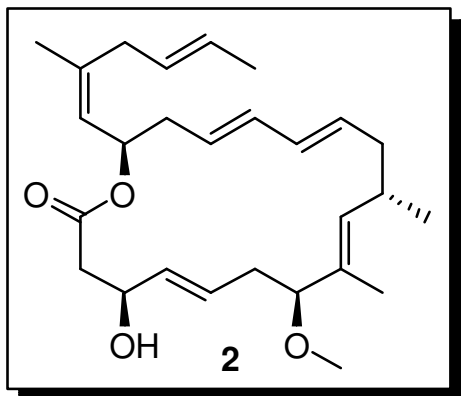

Biselyngbyolide B (2): To an ice cold solution of above macrocycle $27(28 \mathrm{mg}, 0.04 \mathrm{mmol})$ in dry THF $(2 \mathrm{~mL})$ under argon, TBAF (1M solution in THF, $0.05 \mathrm{~mL}, 0.05 \mathrm{mmol})$ was added and the reaction mixture was stirred further for $2 \mathrm{~h}$ at the same temperature prior to quench with saturated $\mathrm{NH}_{4} \mathrm{Cl}$ solution $(1 \mathrm{~mL})$. The resultant mixture was extracted with EtOAC $(2 \times 8 \mathrm{~mL})$, washed with water, brine, dried $\left(\mathrm{Na}_{2} \mathrm{SO}_{4}\right)$, filtered and concentrated in vacuo. Purification by column chromatography $\left(\mathrm{SiO}_{2}, 100-200\right.$ mesh, 25\% EtOAc in hexane as eluant) afforded biselyngbyolide B (2) (17 mg, $96 \%)$ as a colorless oil. $R_{f}=0.17(20 \%$ EtOAc in hexane $) ;[\alpha]_{\mathrm{D}}{ }^{28}=-51.7\left(c 0.48, \mathrm{CHCl}_{3}\right) ;{ }^{1} \mathrm{H}$ $\operatorname{NMR}\left(\mathrm{C}_{6} \mathrm{D}_{6}, 300 \mathrm{MHz}\right) \delta 6.05(\mathrm{dd}, J=14.6,10.5 \mathrm{~Hz}, 1 \mathrm{H}), 5.96(\mathrm{dd}, J=14.6,10.5 \mathrm{~Hz}, 1 \mathrm{H})$, $5.87(\mathrm{dt}, J=9.3,3.6 \mathrm{~Hz}, 1 \mathrm{H}), 5.49-5.32(\mathrm{~m}, 6 \mathrm{H}), 5.28(\mathrm{~d}, J=9.0 \mathrm{~Hz}, 1 \mathrm{H}), 4.88(\mathrm{dd}, J=9.9 \mathrm{~Hz}$, 1H), 4.51-4.45(m, 1H), 3.35(dd, $J=10.5,5.1 \mathrm{~Hz}, 1 \mathrm{H}), 3.08(\mathrm{~s}, 3 \mathrm{H}), 3.05-2.99(\mathrm{~m}, 1 \mathrm{H}), 2.80-$ 2.74(m, 1H), 2.39-2.21(m, 7H), 2.15-2.02(m, 1H), 1.83-1.72(m, 1H), $1.62(\mathrm{~d}, J=1.2 \mathrm{~Hz}, 3 \mathrm{H})$, $1.56(\mathrm{~m}, 3 \mathrm{H}), 1.51(\mathrm{~d}, J=1.2 \mathrm{~Hz}, 3 \mathrm{H}), 0.88(\mathrm{~d}, J=6.8 \mathrm{~Hz}, 3 \mathrm{H}) ;{ }^{13} \mathrm{C} \mathrm{NMR}\left(\mathrm{C}_{6} \mathrm{D}_{6}, 125 \mathrm{MHz}\right) \delta$ 171.1, 139.4, 136.3, 134.2, 133.4, 133.0, 132.9, 130.5, 128.8, 126.2, 124.2, 87.8, 71.1, 69.7, 54.9, 43.5, 40.6, 39.6, 36.1, 35.2, 33.0, 23.3, 22.7, 17.9, 9.7 ppm; IR(neat): $v_{\max } 3384,2926,1730$, $1095 \mathrm{~cm}^{-1}$; HRMS (ESI) $\mathrm{m} / \mathrm{z}$ calculated for $\mathrm{C}_{27} \mathrm{H}_{40} \mathrm{O}_{4} \mathrm{Na}[\mathrm{M}+\mathrm{Na}]^{+} 451.2824$, found 451.2823 . 
5. Copies of ${ }^{1} \mathrm{H}-\mathrm{NMR},{ }^{13} \mathrm{C}-\mathrm{NMR}, 2 \mathrm{D}-\mathrm{NMR}$ and HRMS Spectra

${ }^{1} \mathrm{H}-\mathrm{NMR}$ spectrum of compound $13\left(300 \mathrm{MHz}, \mathrm{CDCl}_{3}\right)$

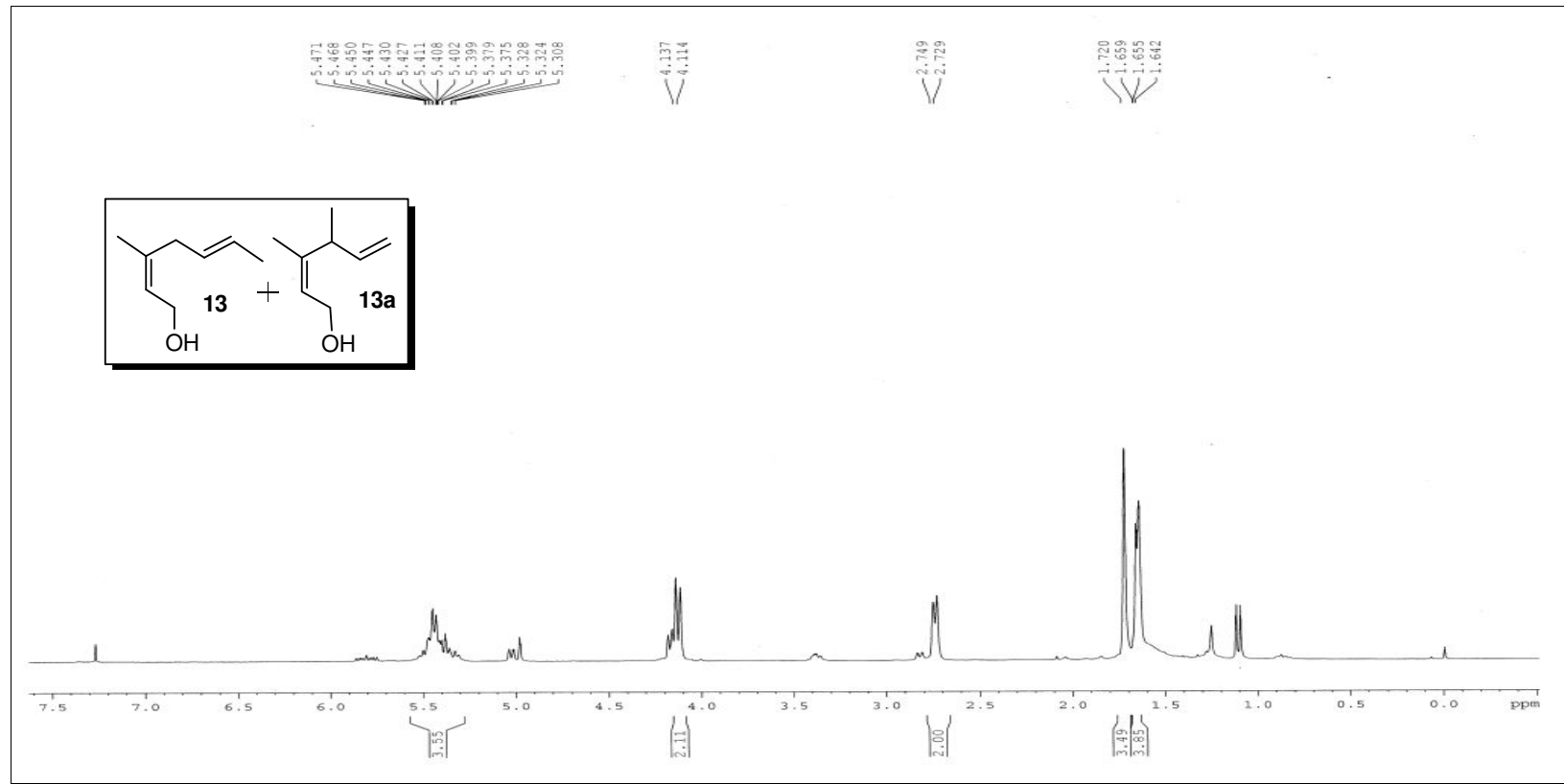

${ }^{13} \mathrm{C}$-NMR spectrum of compound $13\left(75 \mathrm{MHz}, \mathrm{CDCl}_{3}\right)$

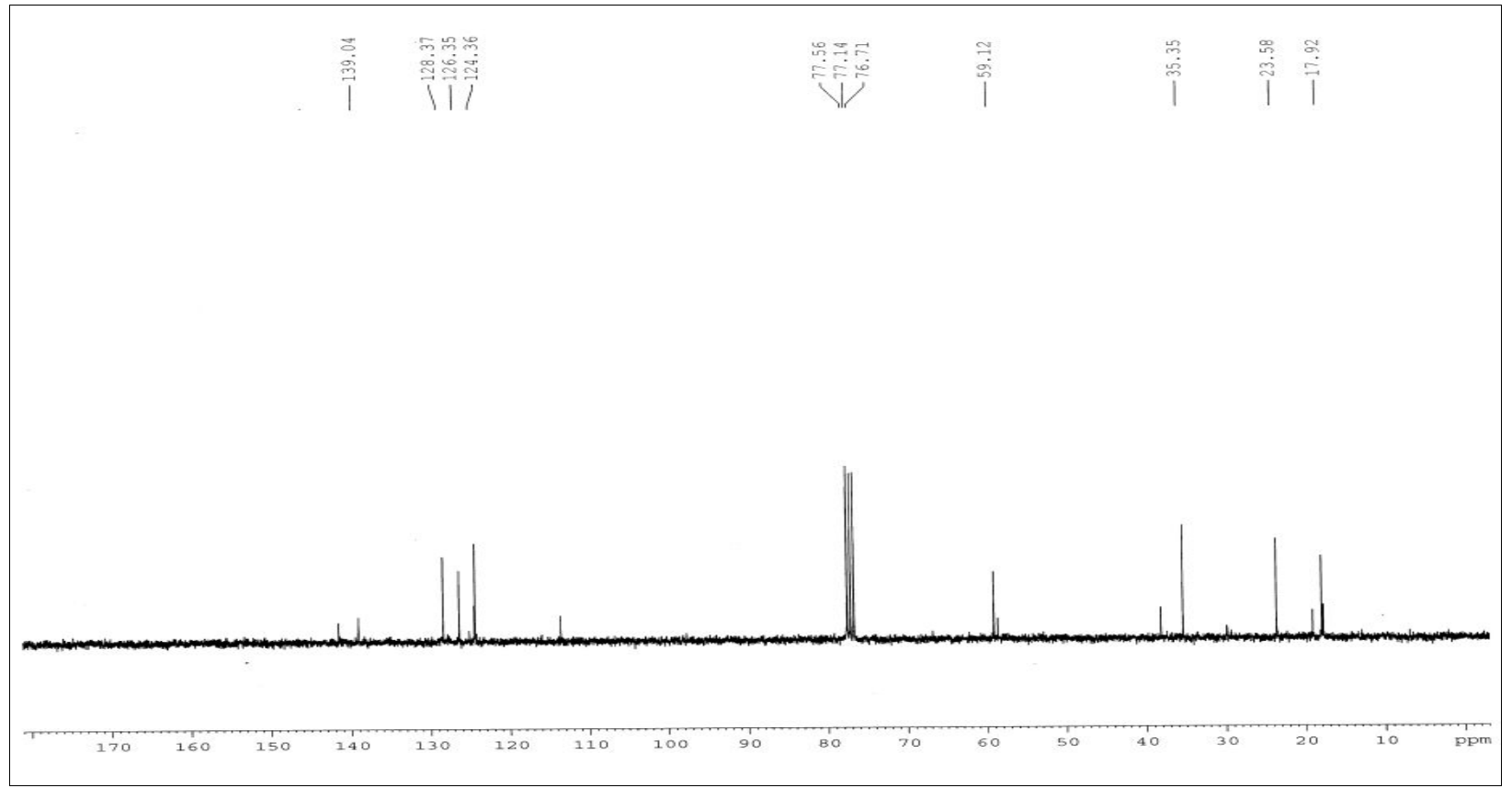


${ }^{1} \mathrm{H}$-NMR spectrum of compound $8\left(300 \mathrm{MHz}, \mathrm{CDCl}_{3}\right)$

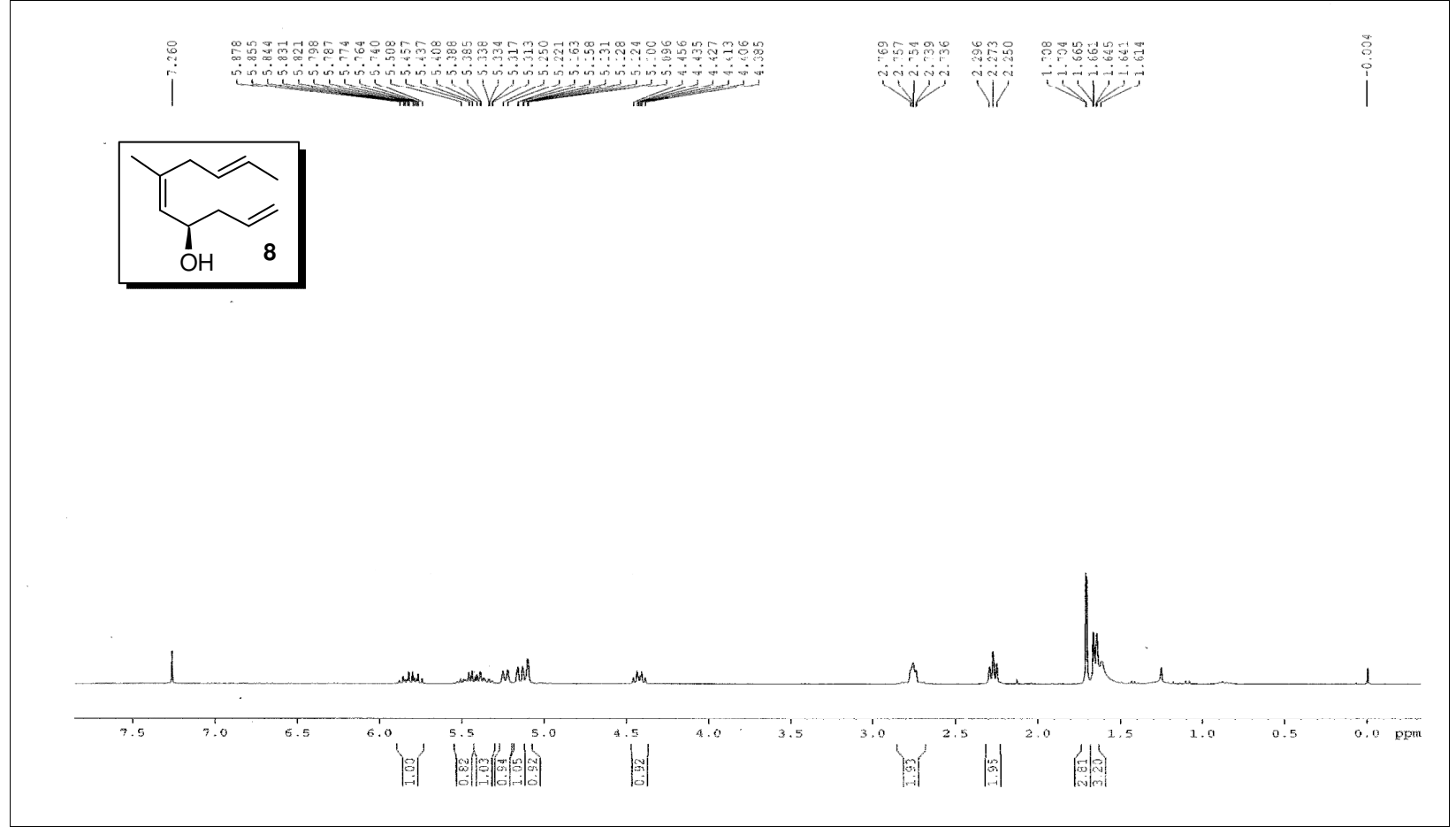

${ }^{13} \mathrm{C}$-NMR spectrum of compound $8\left(75 \mathrm{MHz}, \mathrm{CDCl}_{3}\right)$

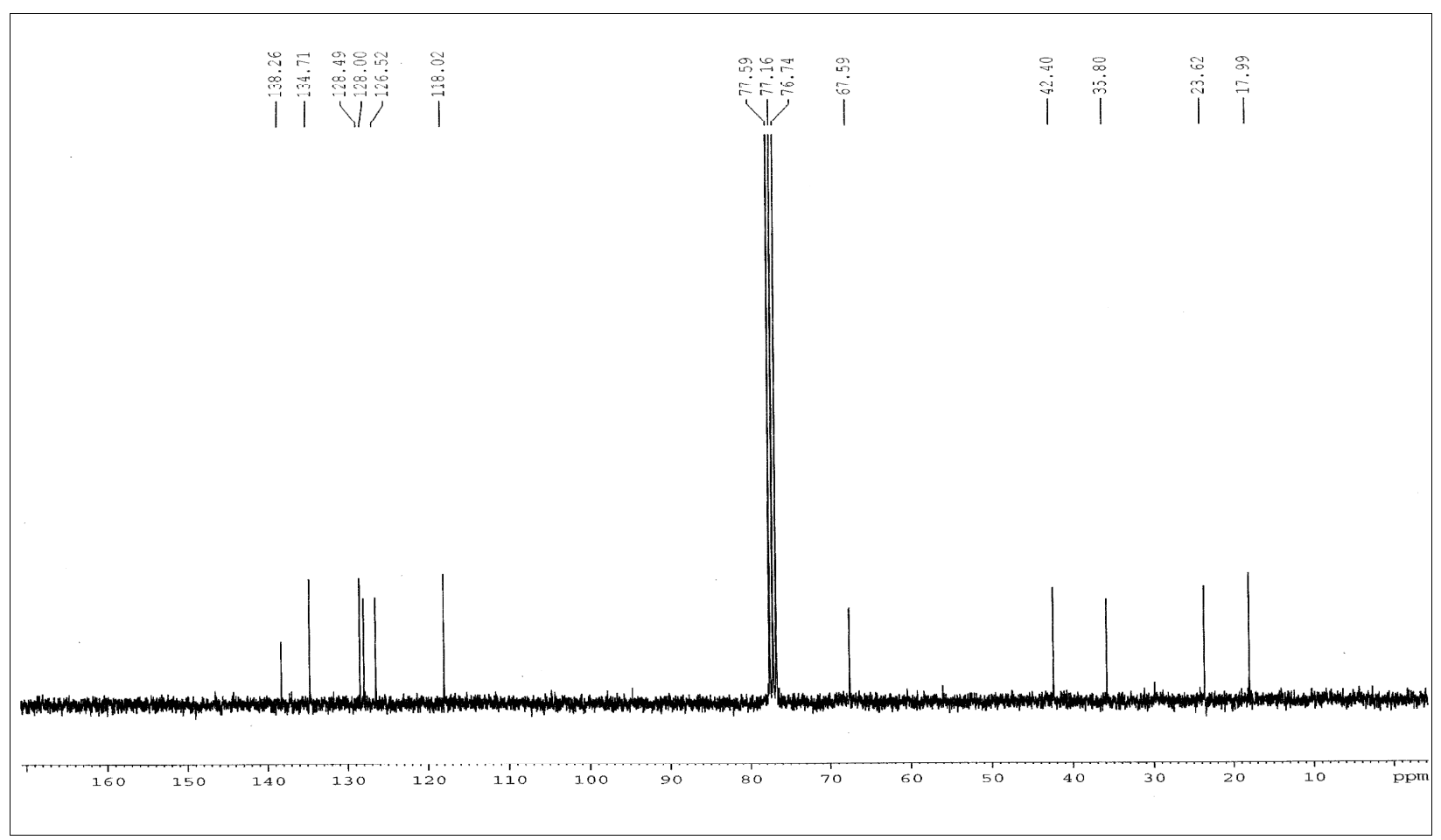


${ }^{1} \mathrm{H}-\mathrm{NMR}$ spectrum of compound $15 \& 15 \mathrm{a}\left(300 \mathrm{MHz}, \mathrm{CDCl}_{3}\right)$

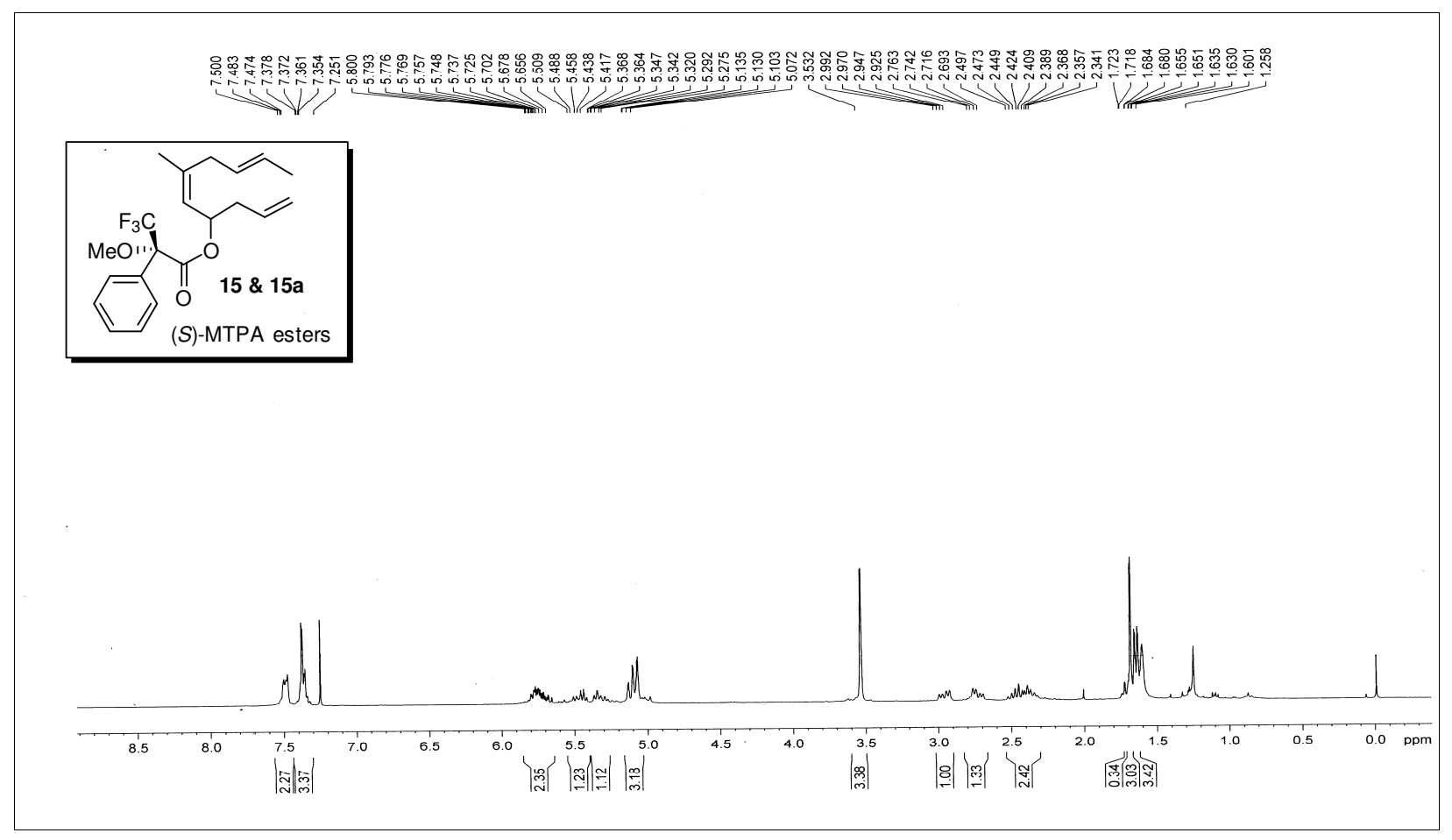

${ }^{13} \mathrm{C}-\mathrm{NMR}$ spectrum of compound $15 \& 15 \mathrm{a}\left(75 \mathrm{MHz}, \mathrm{CDCl}_{3}\right)$

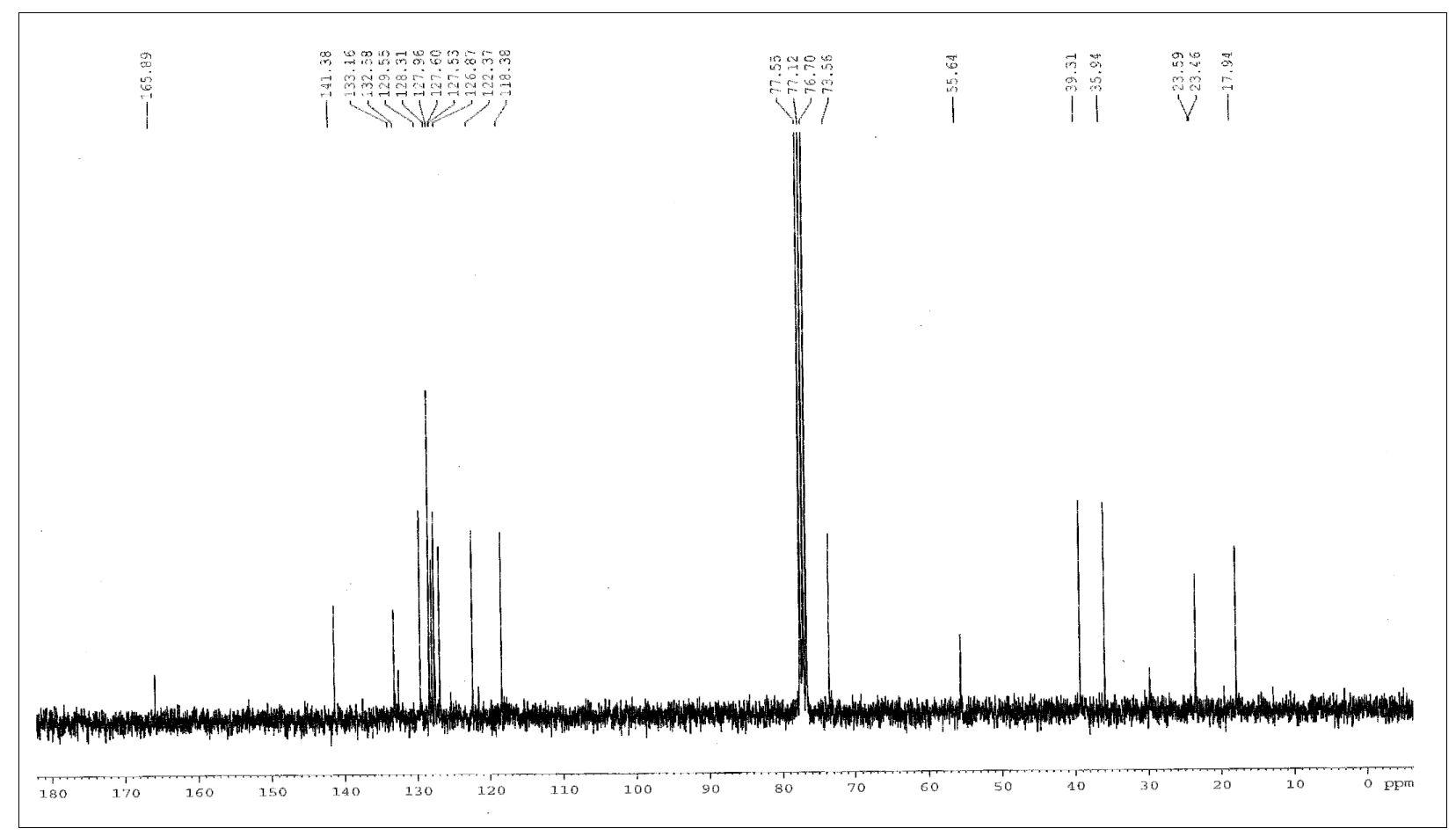


${ }^{1} \mathrm{H}$-NMR spectrum of compound $17\left(300 \mathrm{MHz}, \mathrm{CDCl}_{3}\right)$

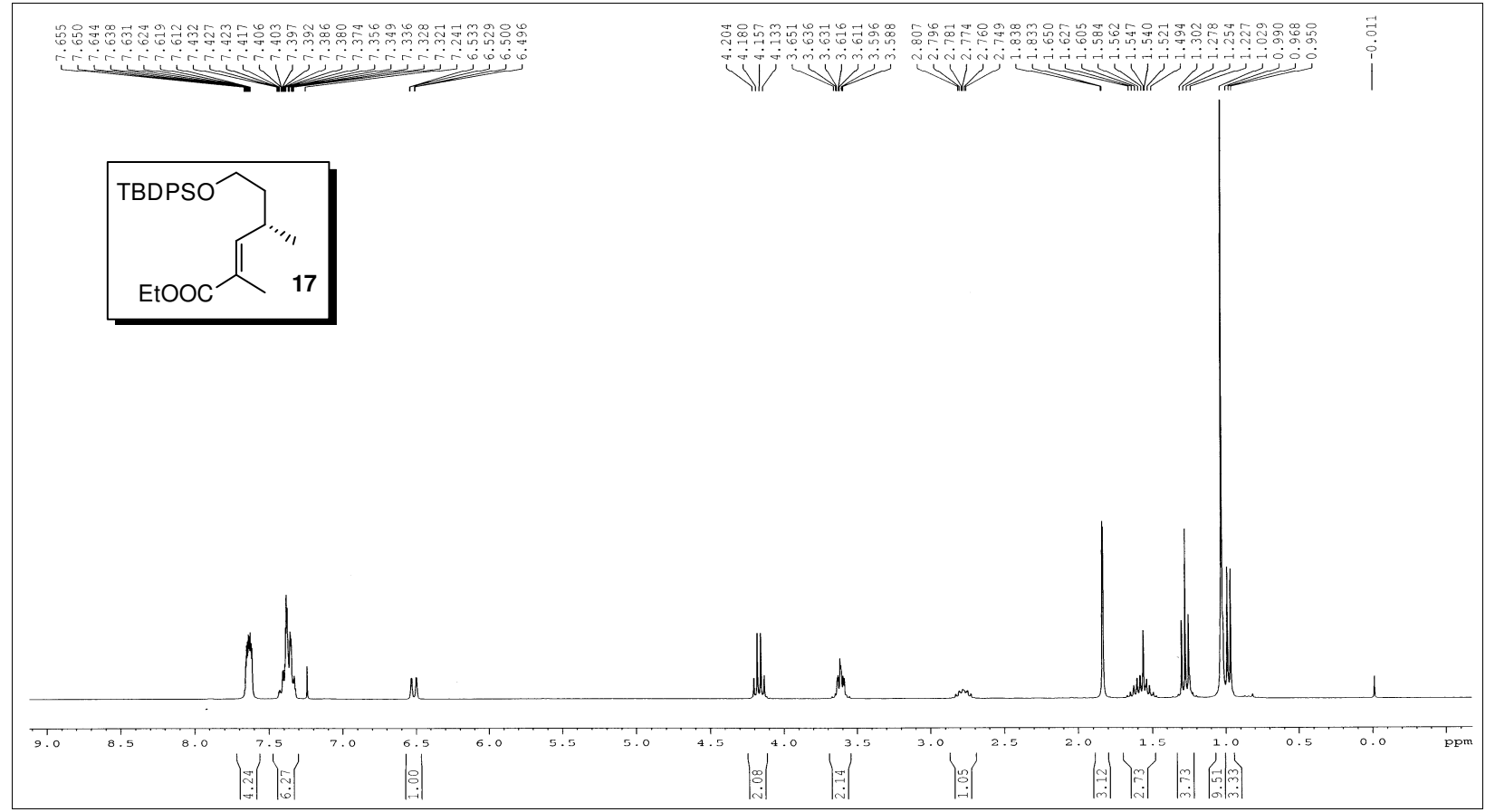

${ }^{13} \mathrm{C}$-NMR spectrum of compound $17\left(75 \mathrm{MHz}, \mathrm{CDCl}_{3}\right)$

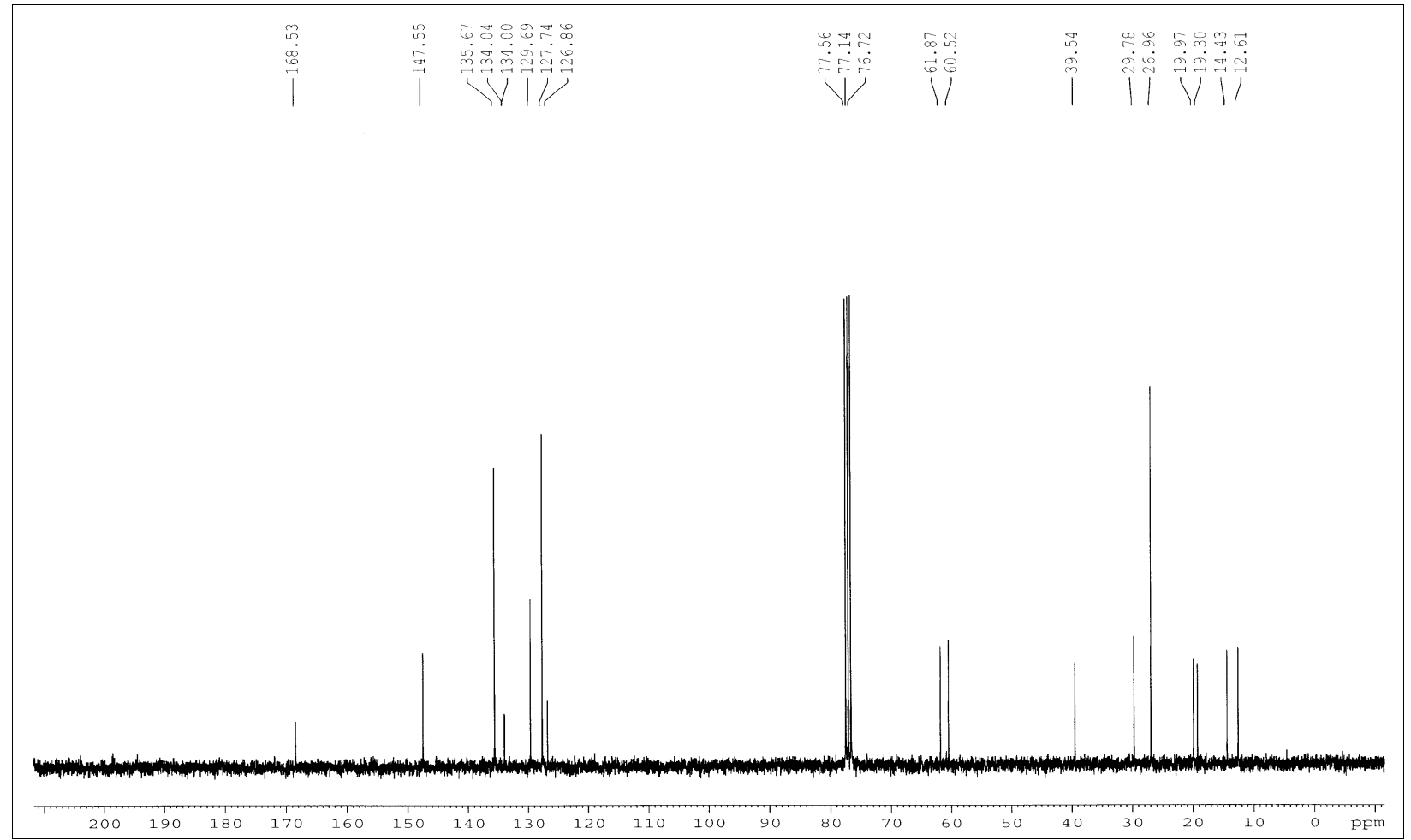


${ }^{1} \mathrm{H}-\mathrm{NMR}$ spectrum of compound $19\left(300 \mathrm{MHz}, \mathrm{CDCl}_{3}\right)$

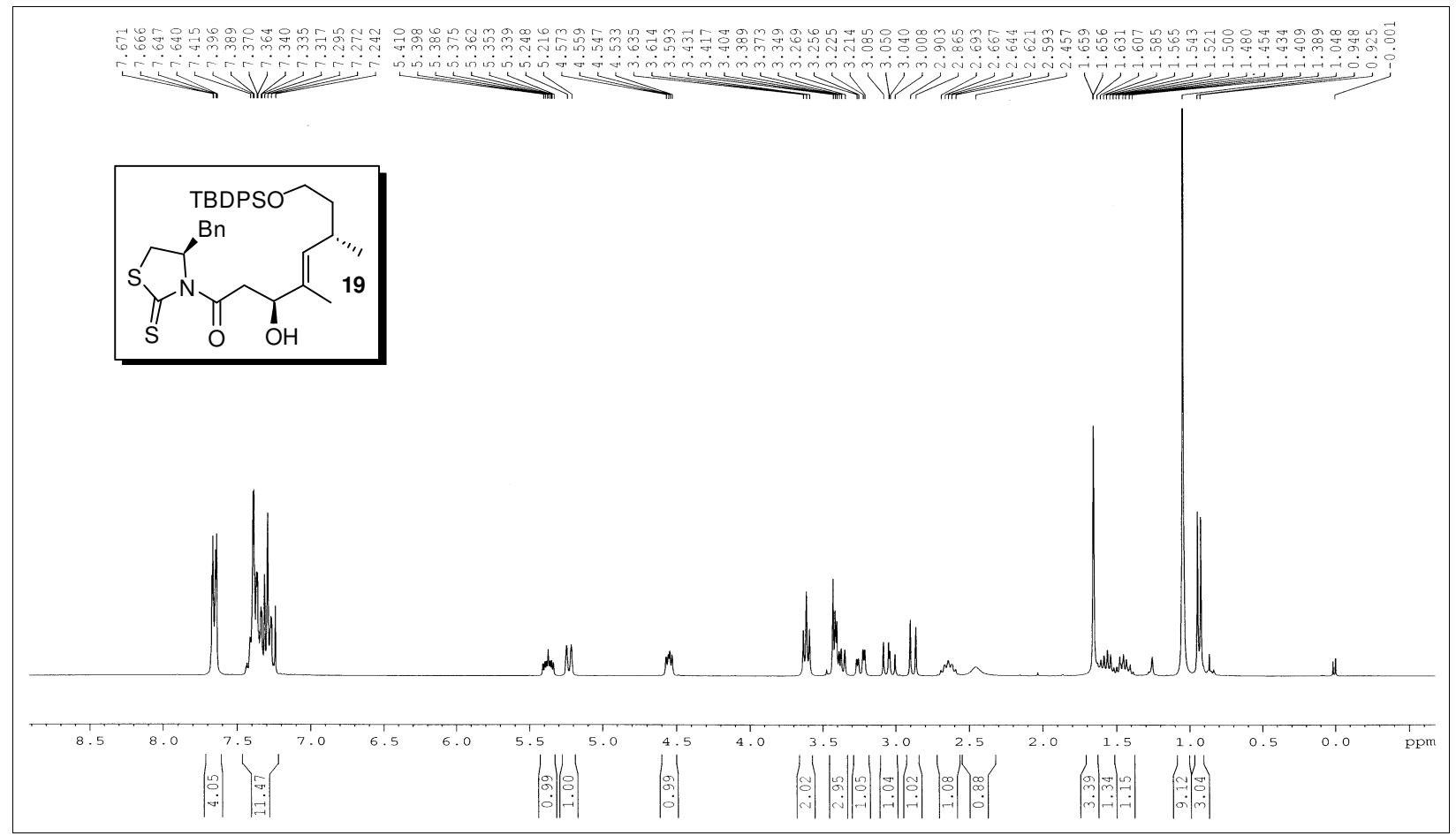

${ }^{13} \mathrm{C}$-NMR spectrum of compound $19\left(75 \mathrm{MHz}, \mathrm{CDCl}_{3}\right)$

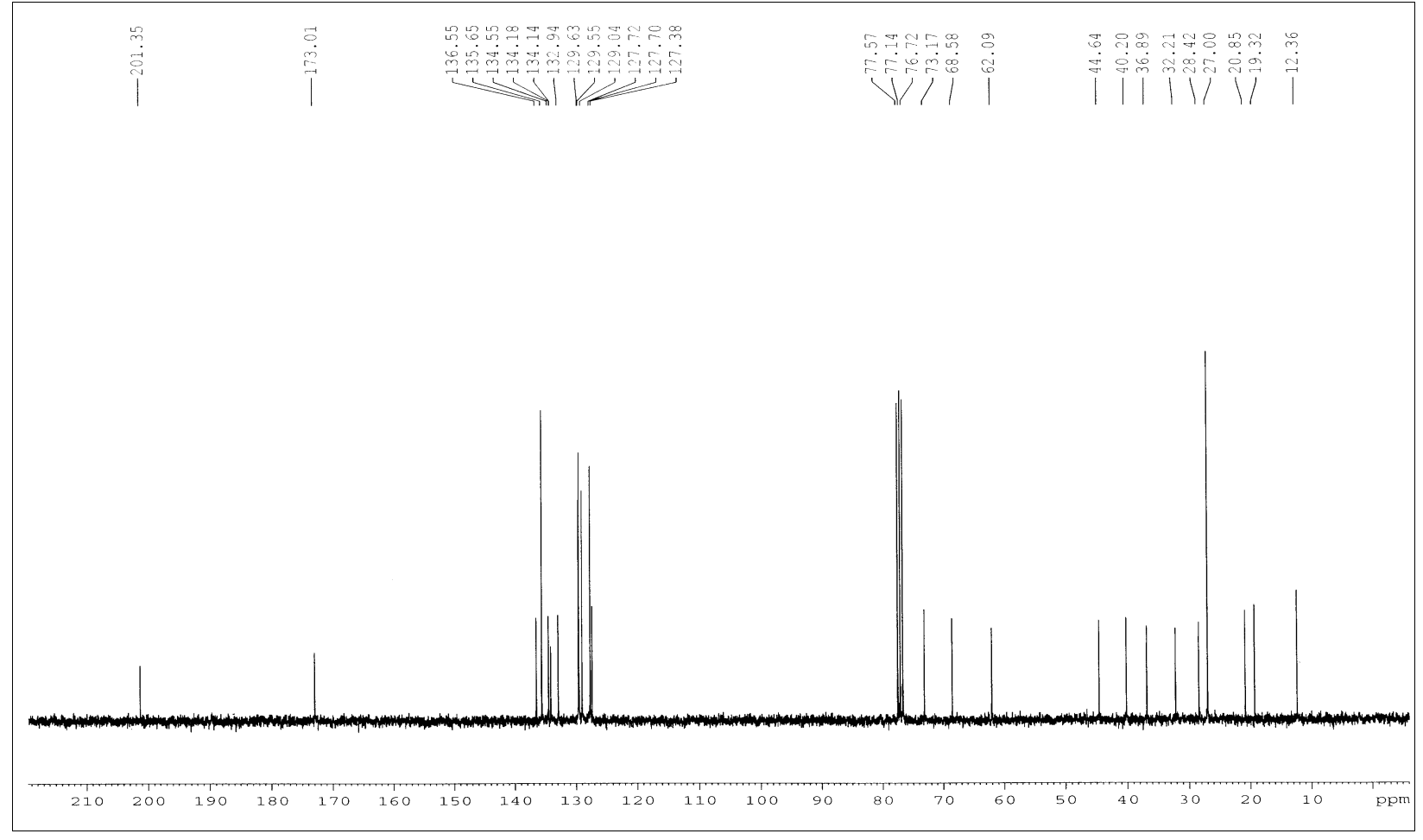

S28 
${ }^{1} \mathrm{H}$-NMR spectrum of compound $20\left(300 \mathrm{MHz}, \mathrm{CDCl}_{3}\right)$

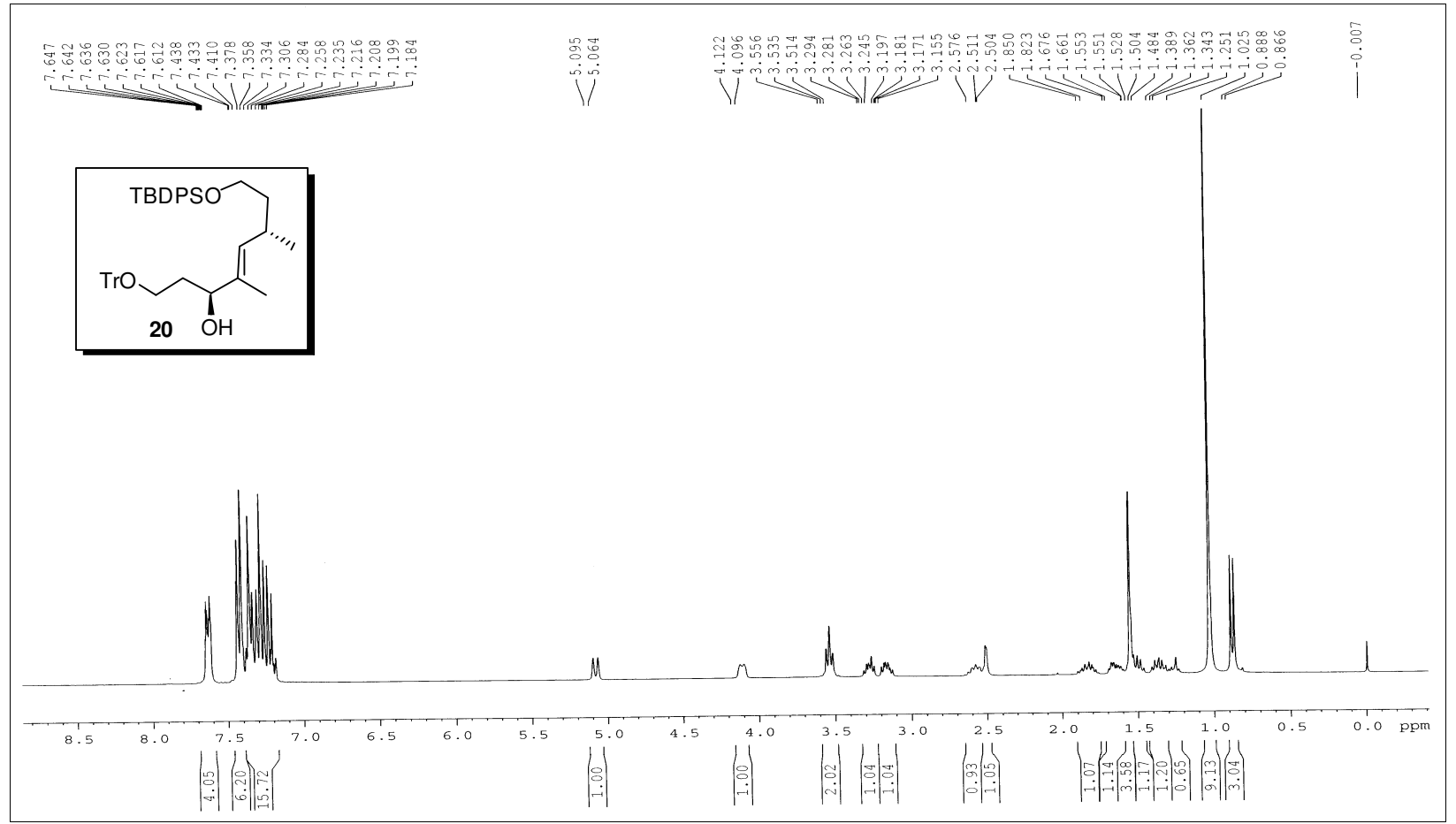

${ }^{13} \mathrm{C}$-NMR spectrum of compound $20\left(75 \mathrm{MHz}, \mathrm{CDCl}_{3}\right)$

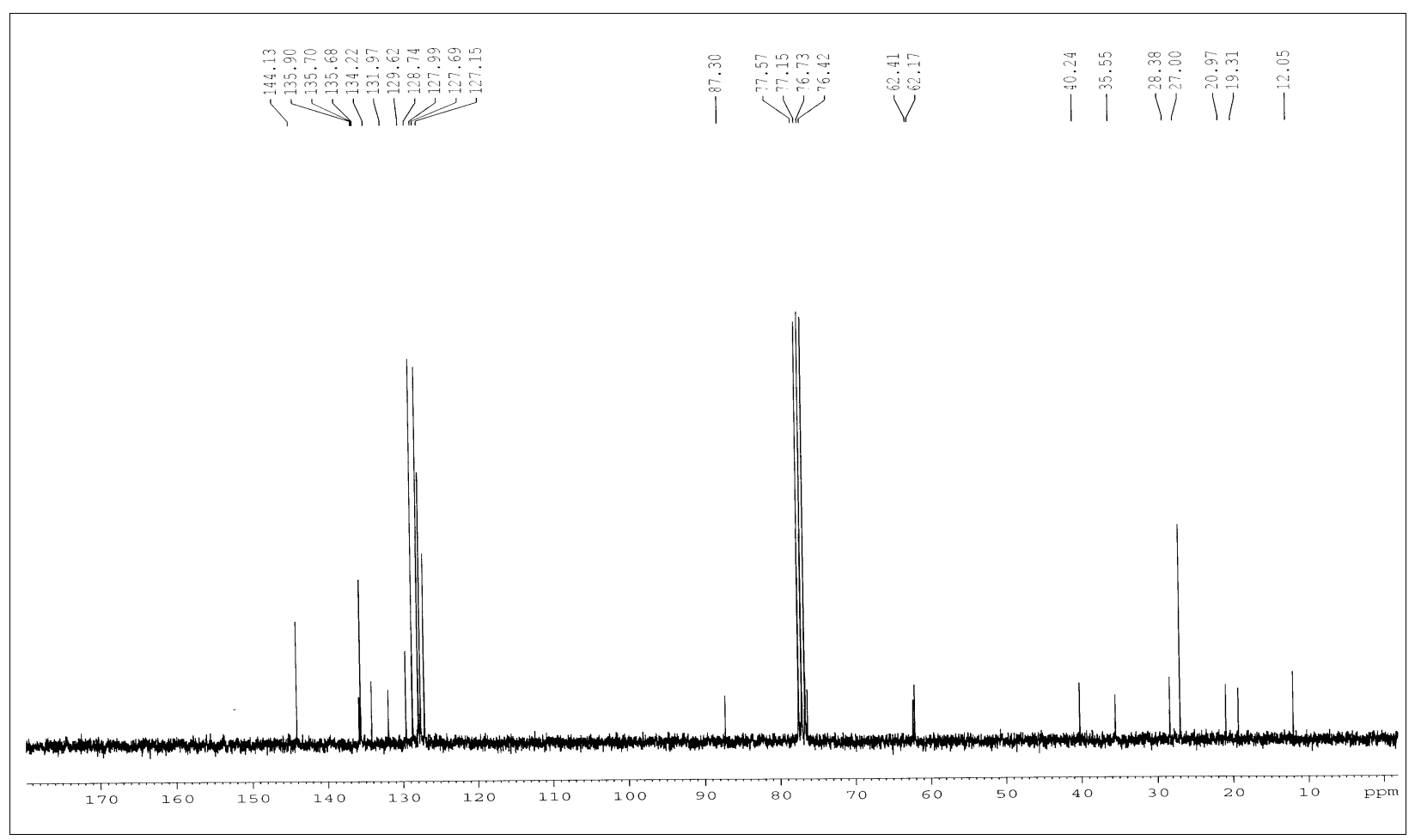


${ }^{1} \mathrm{H}-\mathrm{NMR}$ spectrum of compound $21\left(300 \mathrm{MHz}, \mathrm{CDCl}_{3}\right)$

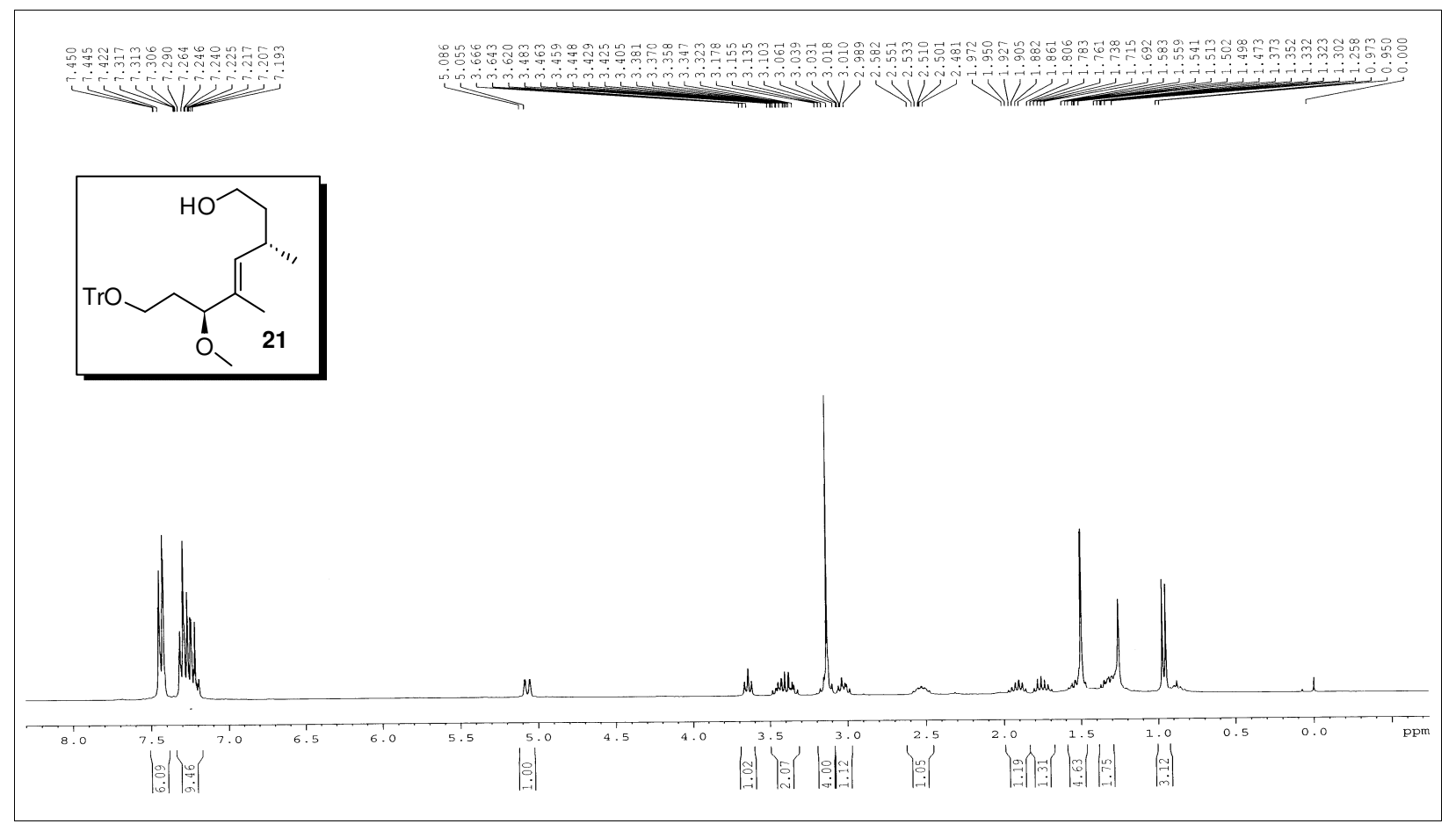

\section{${ }^{13} \mathrm{C}$-NMR spectrum of compound $21\left(75 \mathrm{MHz}, \mathrm{CDCl}_{3}\right)$}

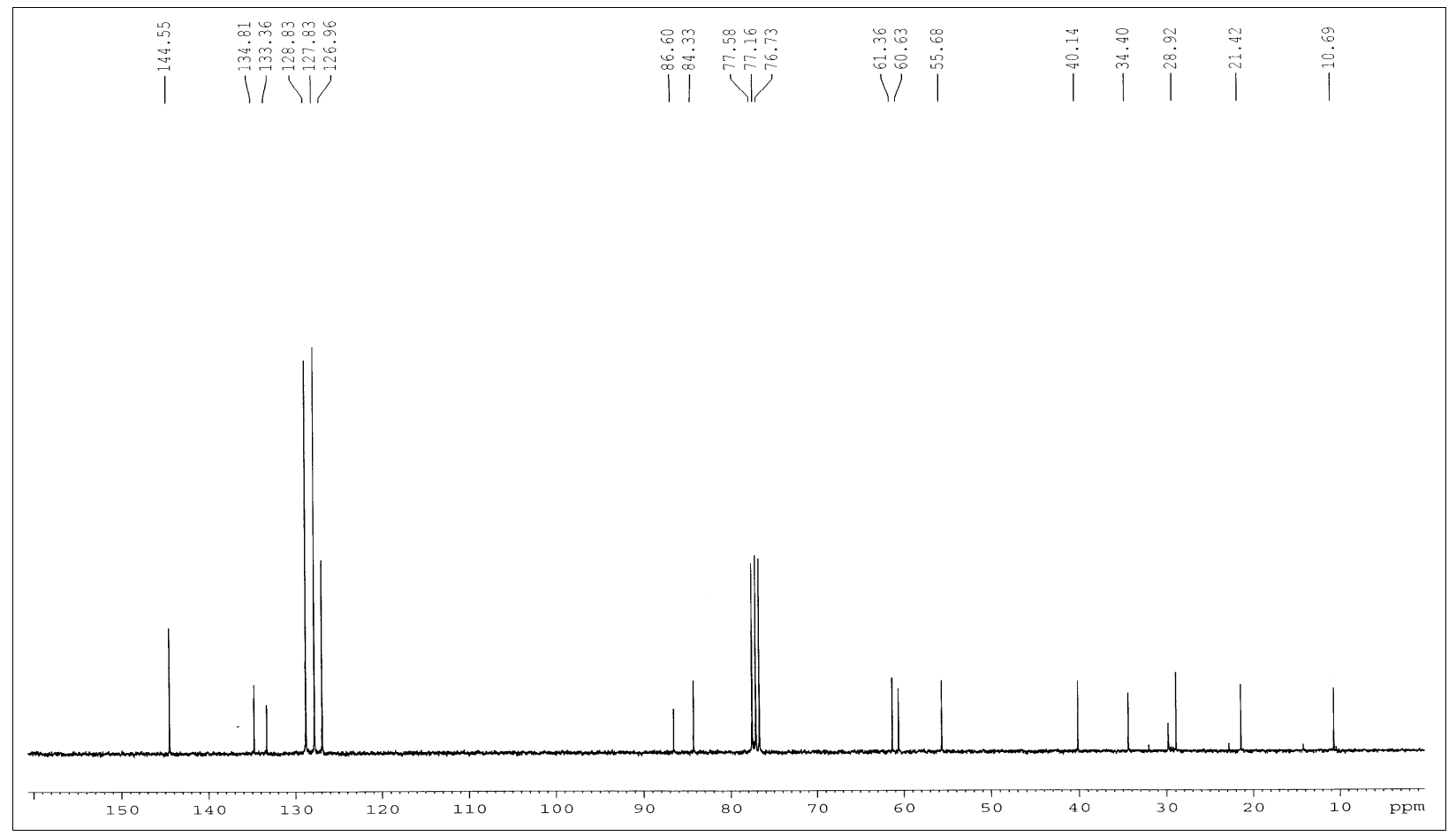

S30 
${ }^{1} \mathrm{H}-\mathrm{NMR}$ spectrum of compound $22\left(500 \mathrm{MHz}, \mathrm{CDCl}_{3}\right)$

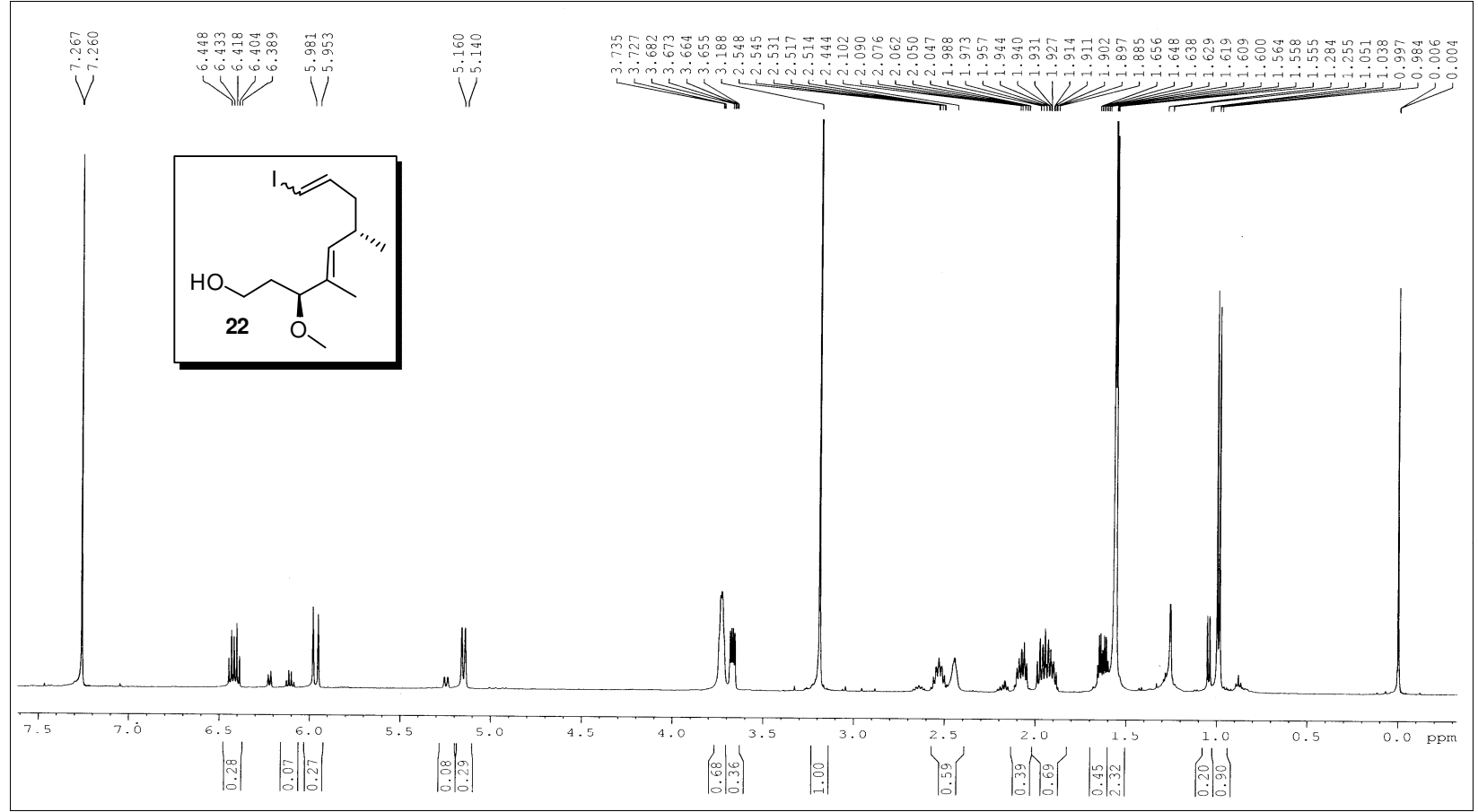

${ }^{13} \mathrm{C}$-NMR spectrum of compound $22\left(75 \mathrm{MHz}, \mathrm{CDCl}_{3}\right)$

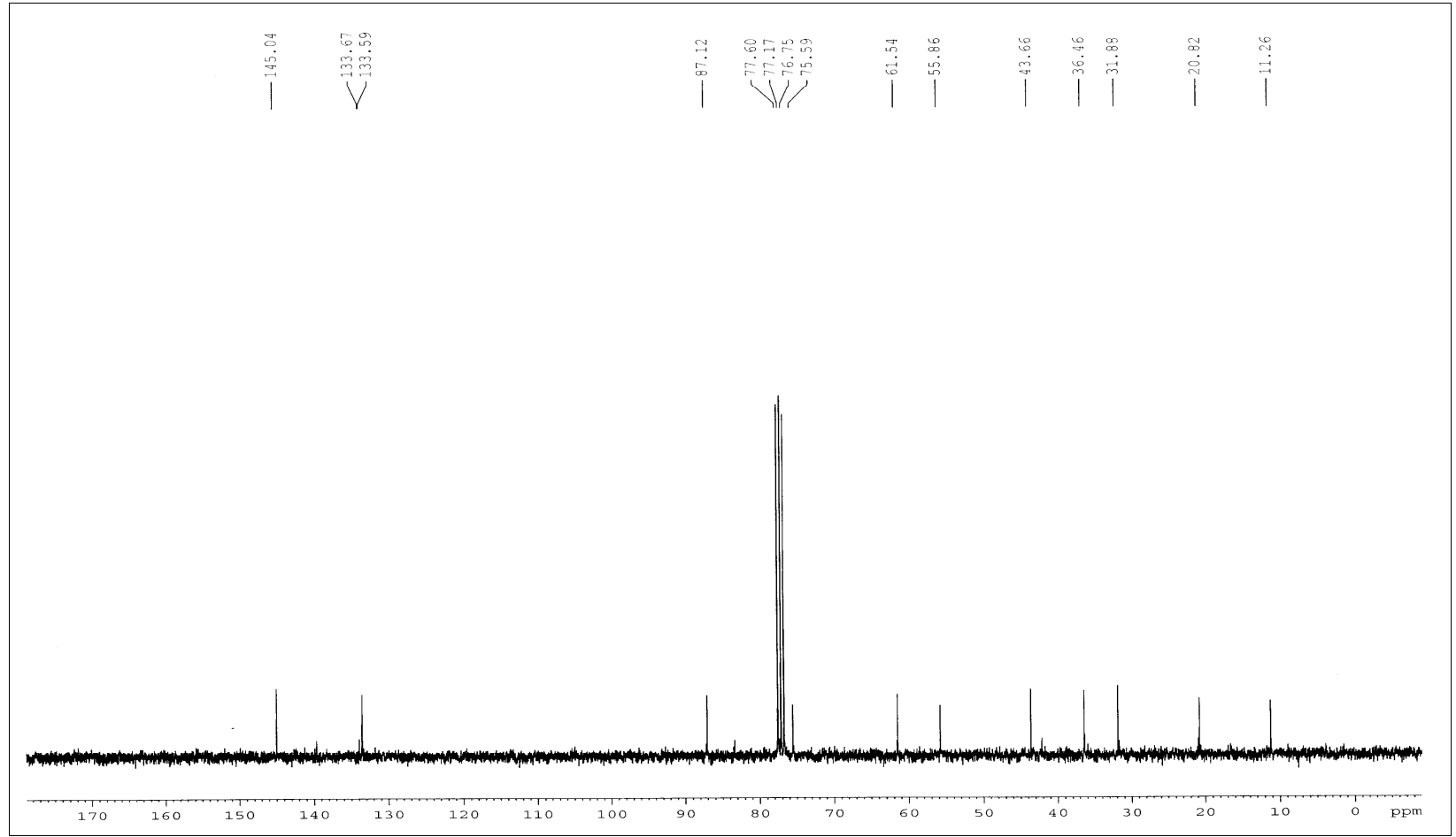

S31 
${ }^{1} \mathrm{H}$-NMR spectrum of compound $10\left(400 \mathrm{MHz}, \mathrm{CDCl}_{3}\right)$

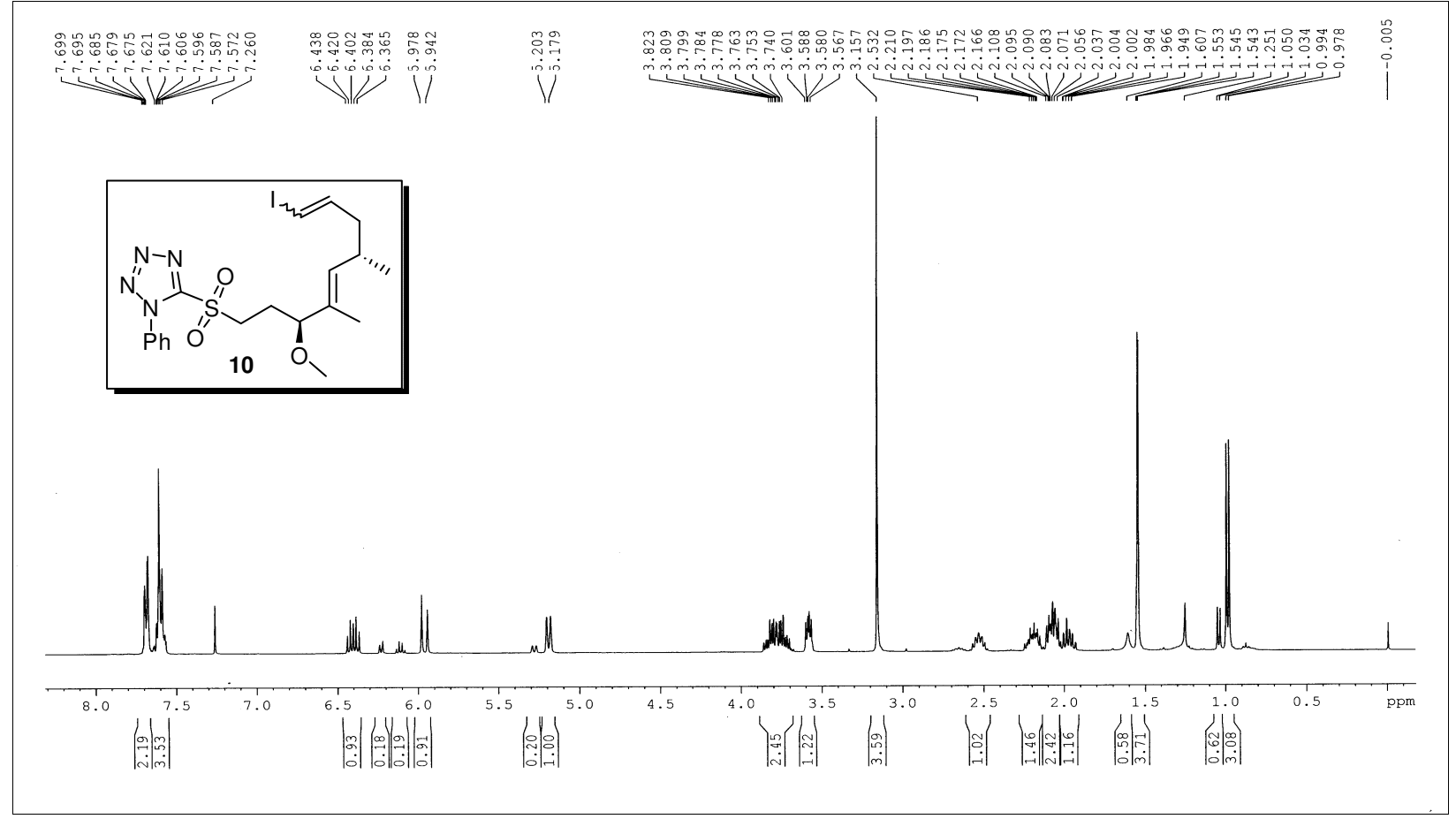

${ }^{13} \mathrm{C}$-NMR spectrum of compound $10\left(75 \mathrm{MHz}, \mathrm{CDCl}_{3}\right)$

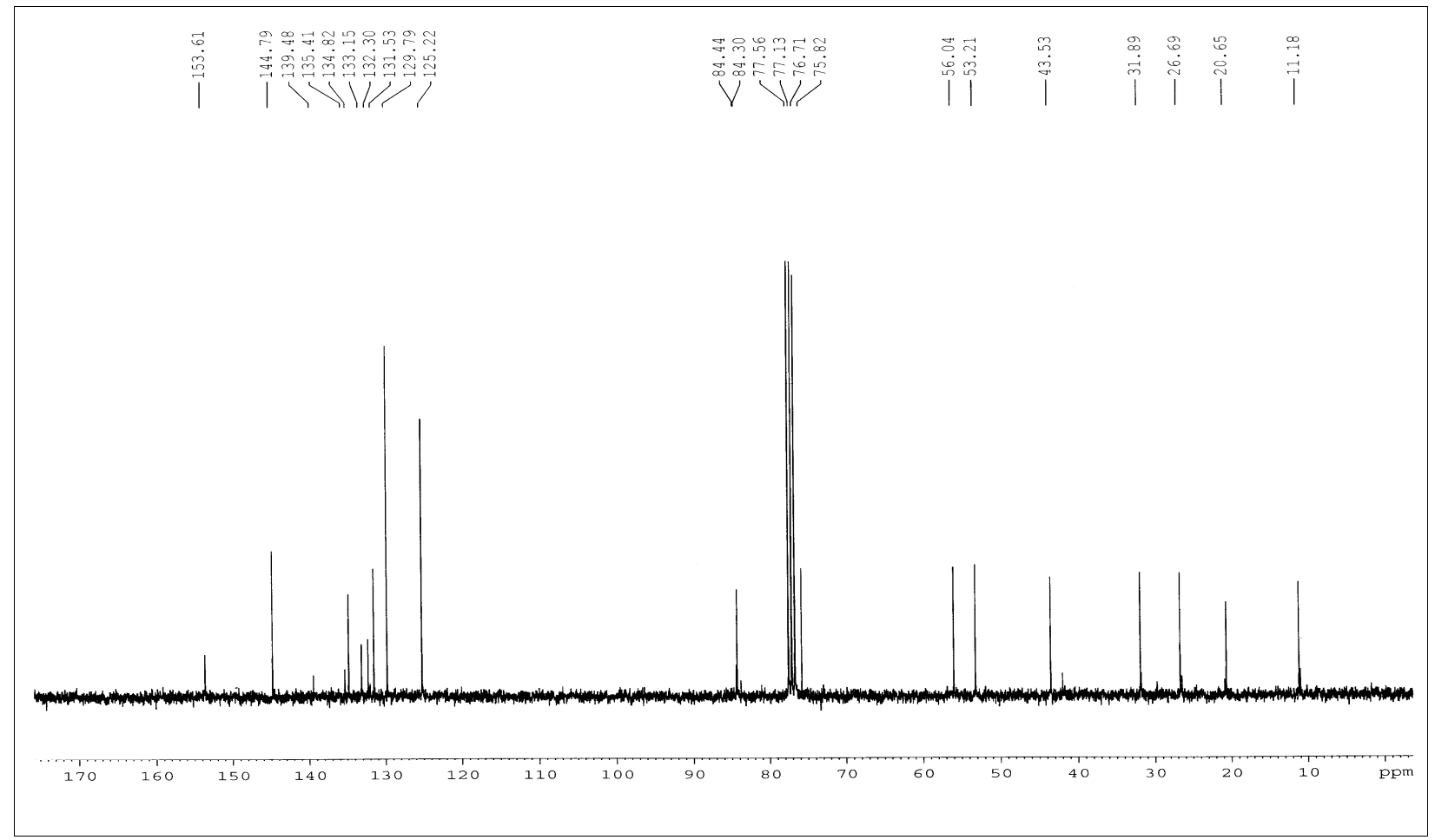


${ }^{1} \mathrm{H}-\mathrm{NMR}$ spectrum of compound 25 (300 $\left.\mathrm{MHz}, \mathrm{CDCl}_{3}\right)$

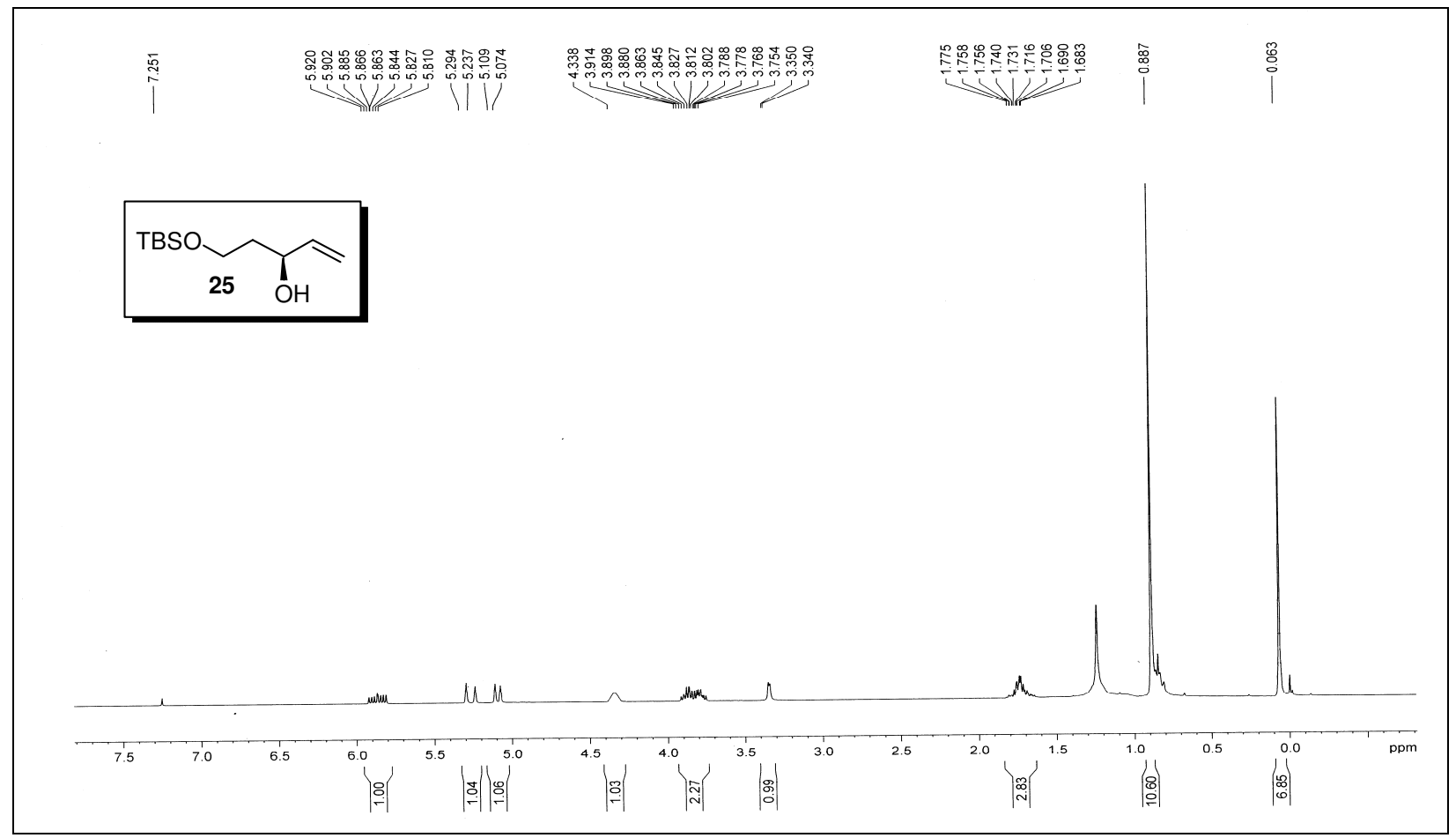

${ }^{13} \mathrm{C}$-NMR spectrum of compound $25\left(75 \mathrm{MHz}, \mathrm{CDCl}_{3}\right)$

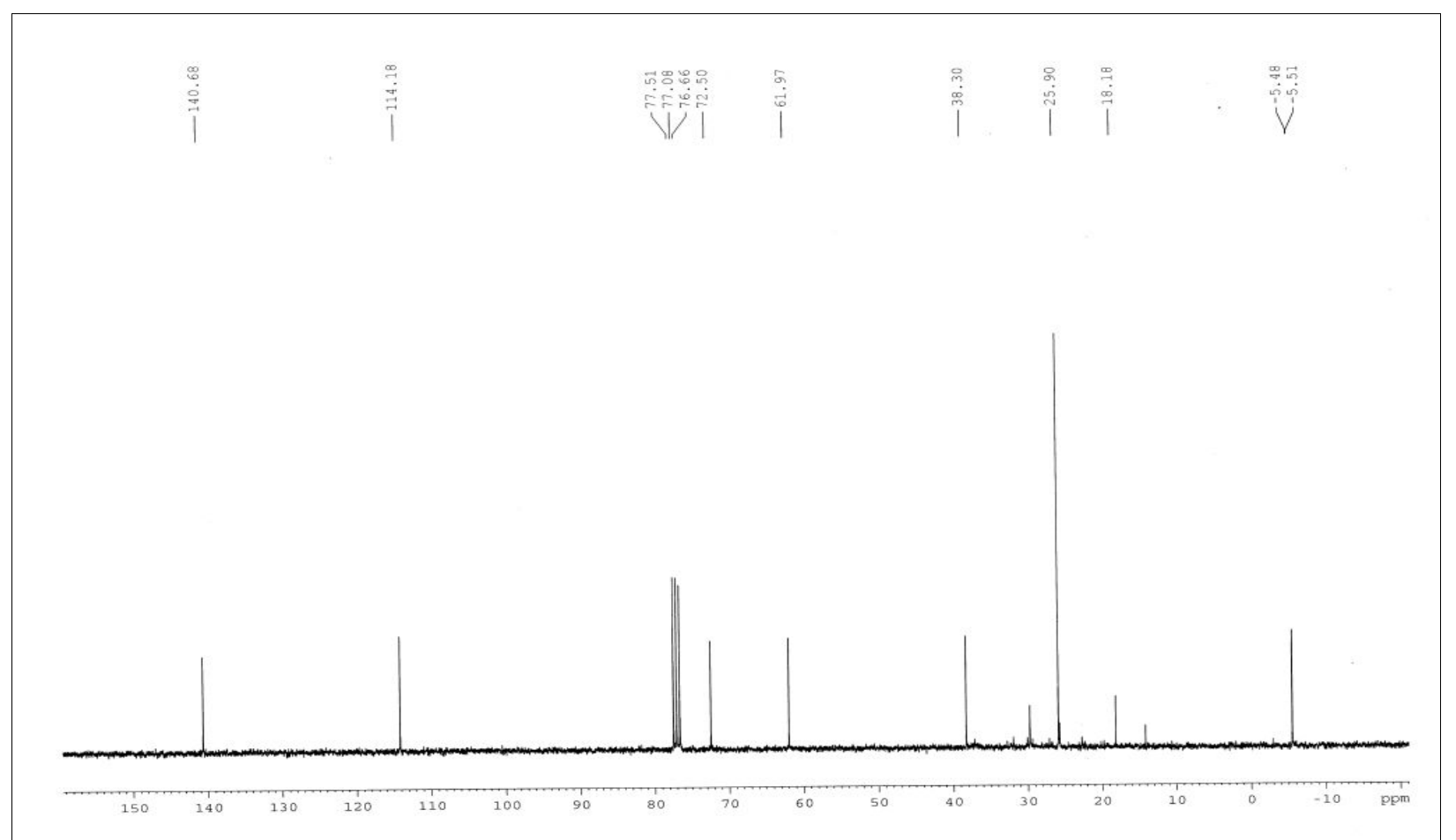

S33 
${ }^{1} \mathrm{H}$-NMR spectrum of compound $26\left(300 \mathrm{MHz}, \mathrm{CDCl}_{3}\right)$

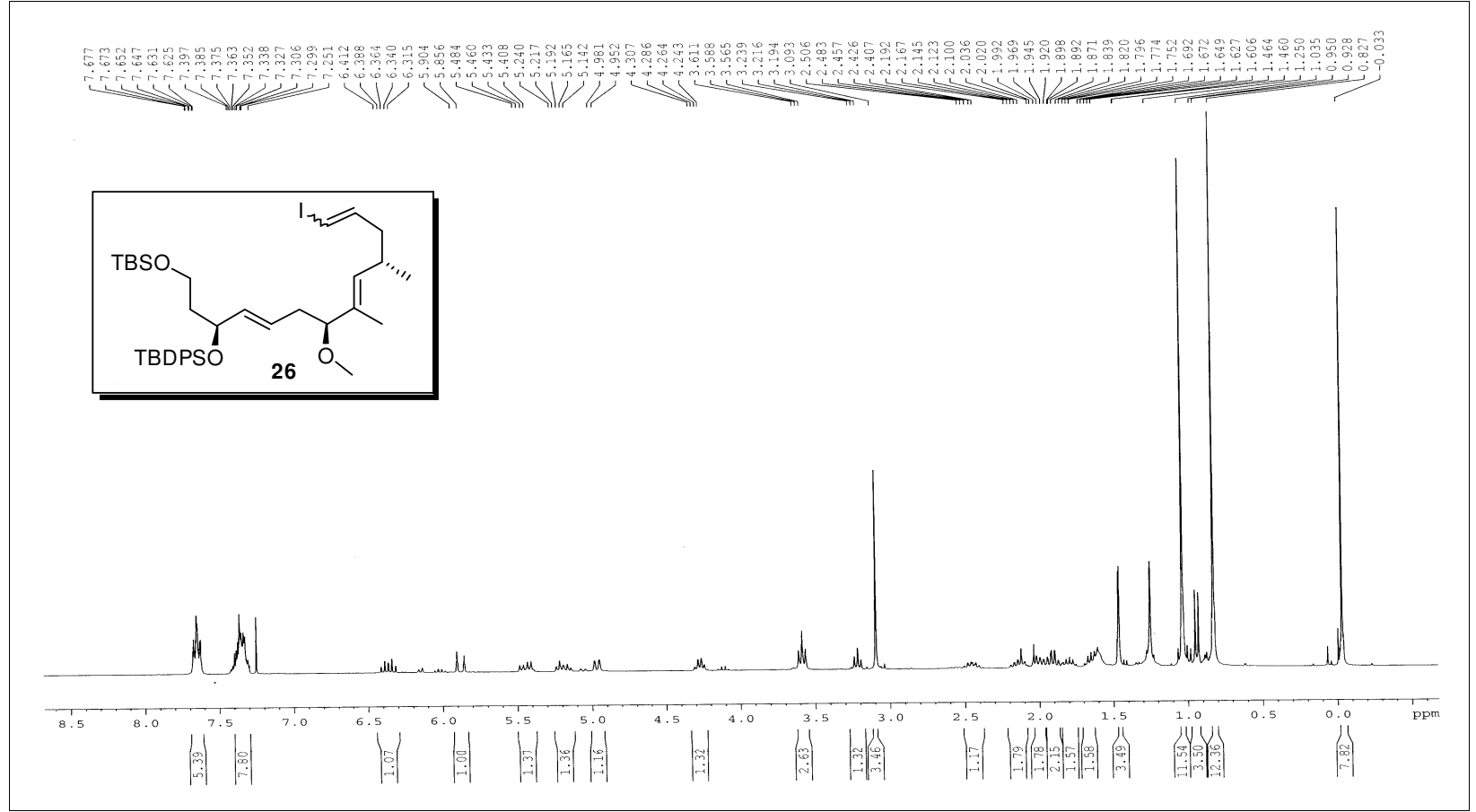

${ }^{13} \mathrm{C}$-NMR spectrum of compound $26\left(75 \mathrm{MHz}, \mathrm{CDCl}_{3}\right)$

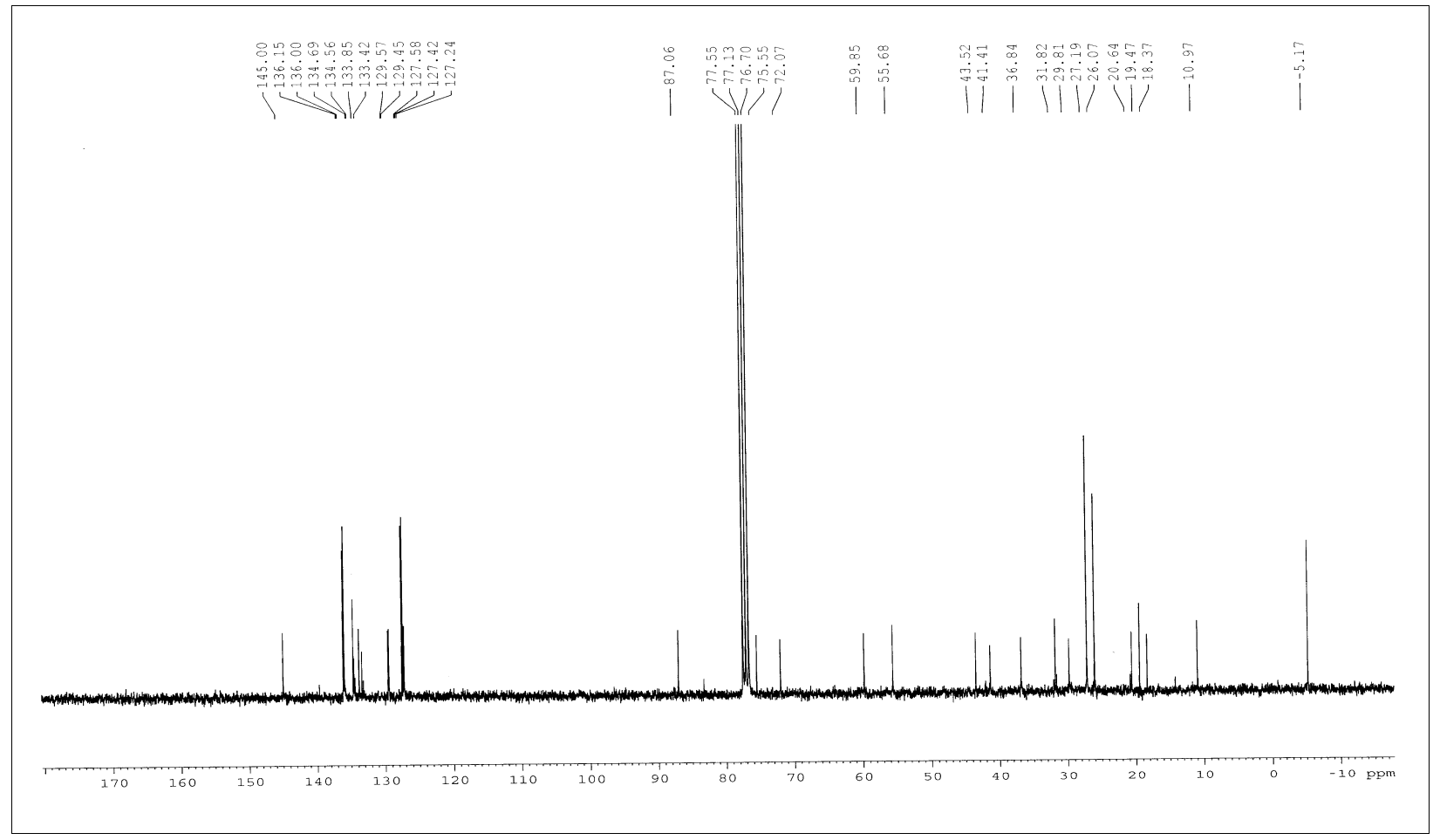


${ }^{1} \mathrm{H}$-NMR spectrum of compound $9\left(300 \mathrm{MHz}, \mathrm{CDCl}_{3}\right)$

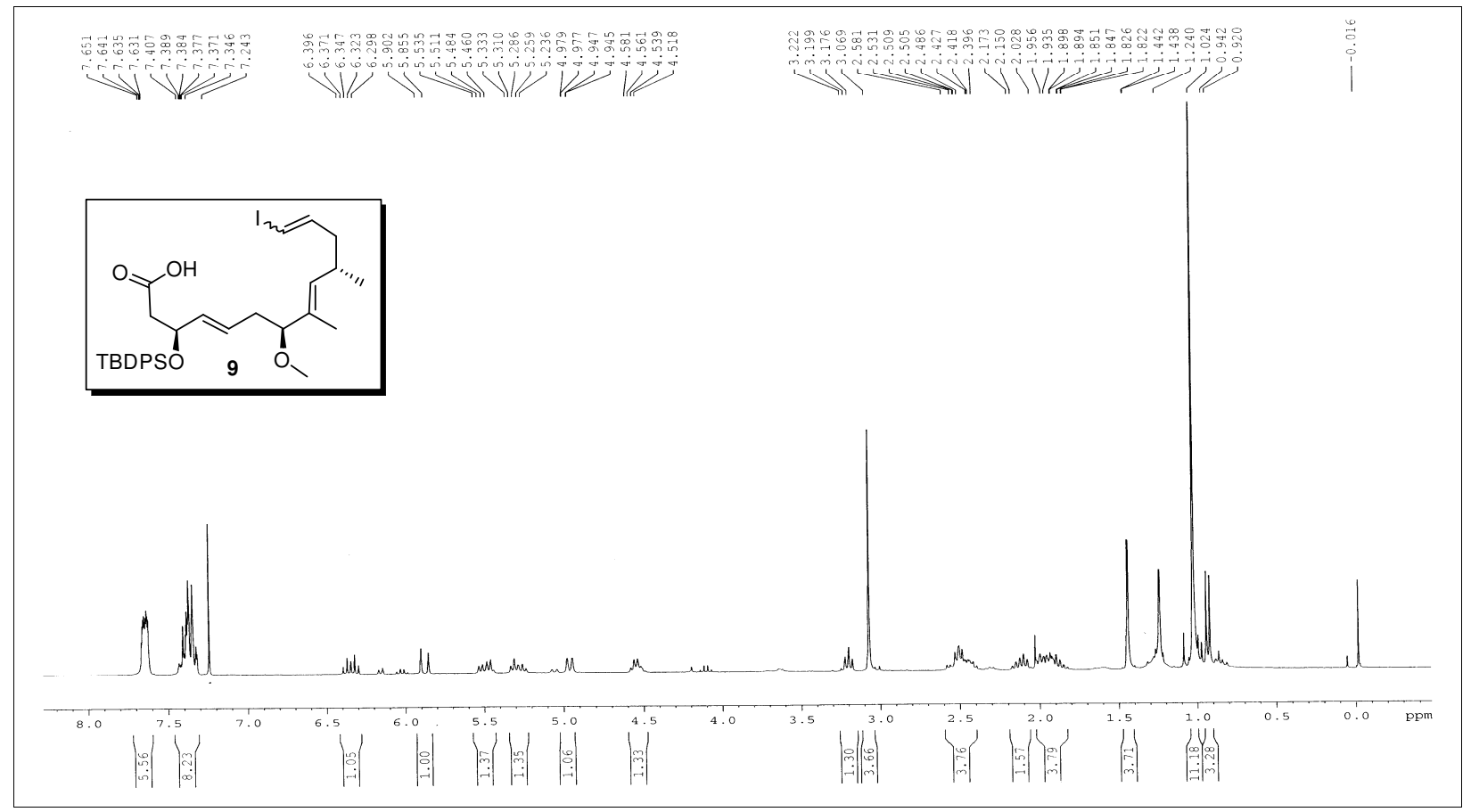

${ }^{13} \mathrm{C}$-NMR spectrum of compound $9\left(75 \mathrm{MHz}, \mathrm{CDCl}_{3}\right)$

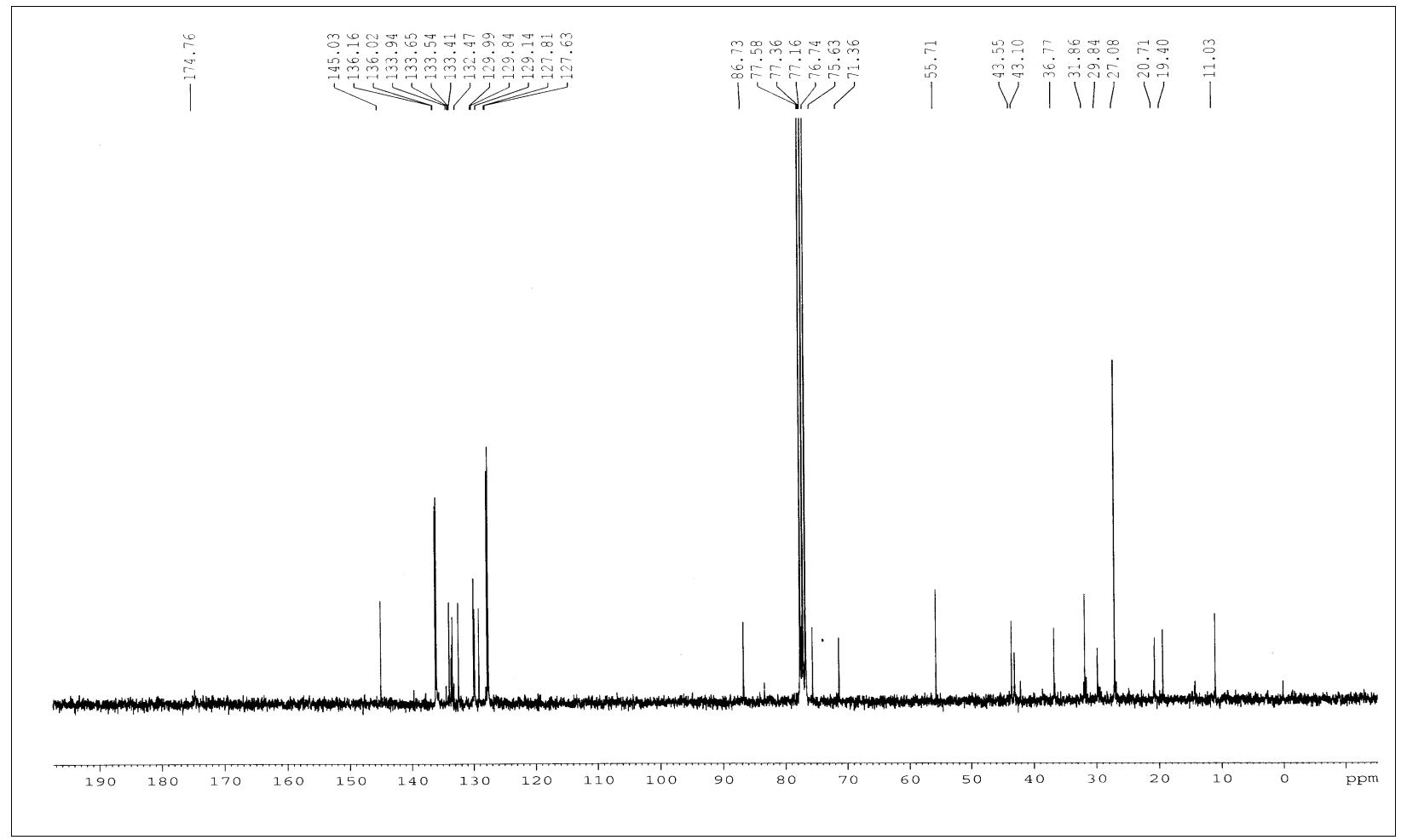

S35 
${ }^{1} \mathrm{H}$-NMR spectrum of compound $7\left(300 \mathrm{MHz}, \mathrm{CDCl}_{3}\right)$

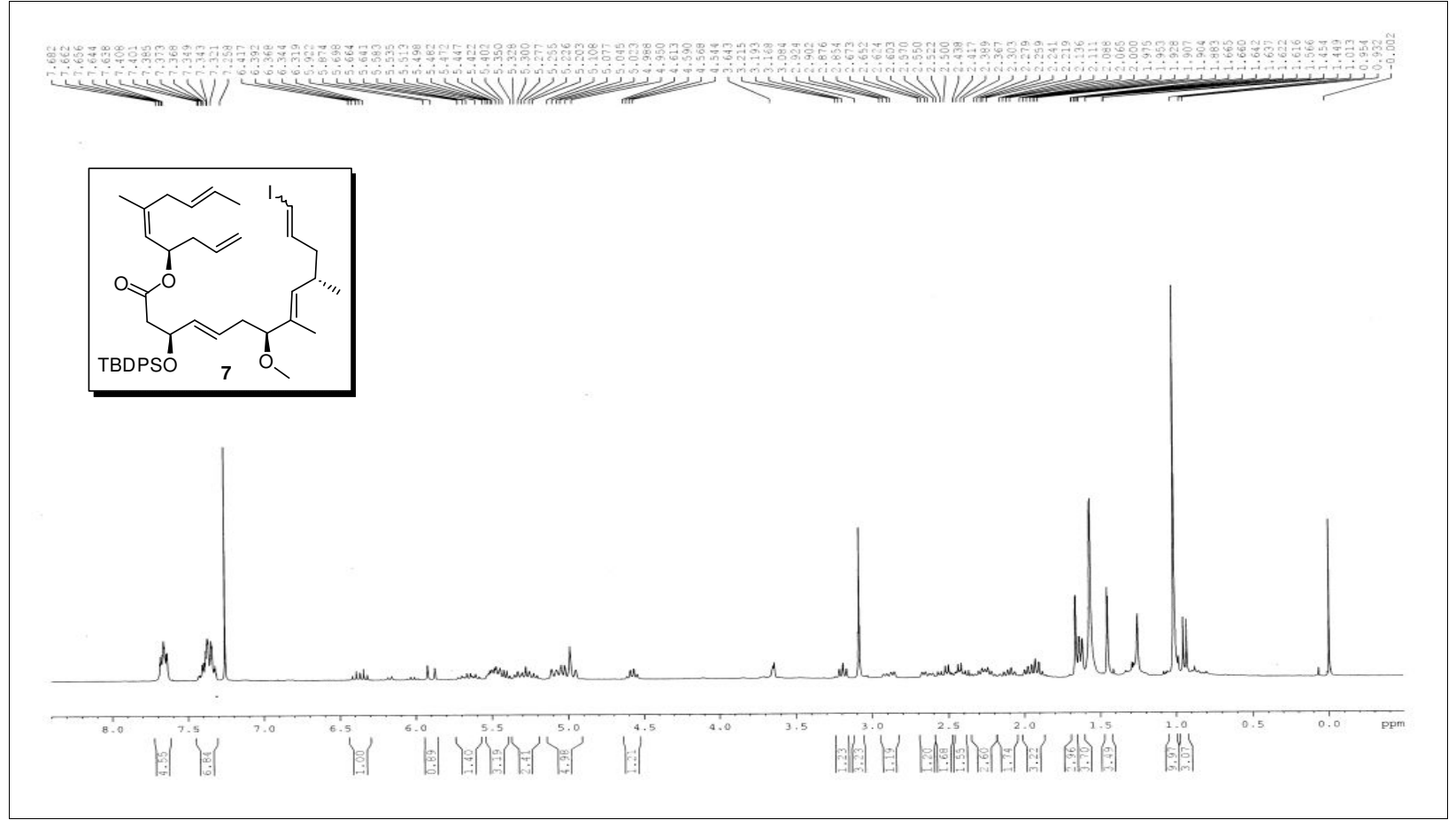

${ }^{13} \mathrm{C}$-NMR spectrum of compound $7\left(75 \mathrm{MHz}, \mathrm{CDCl}_{3}\right)$

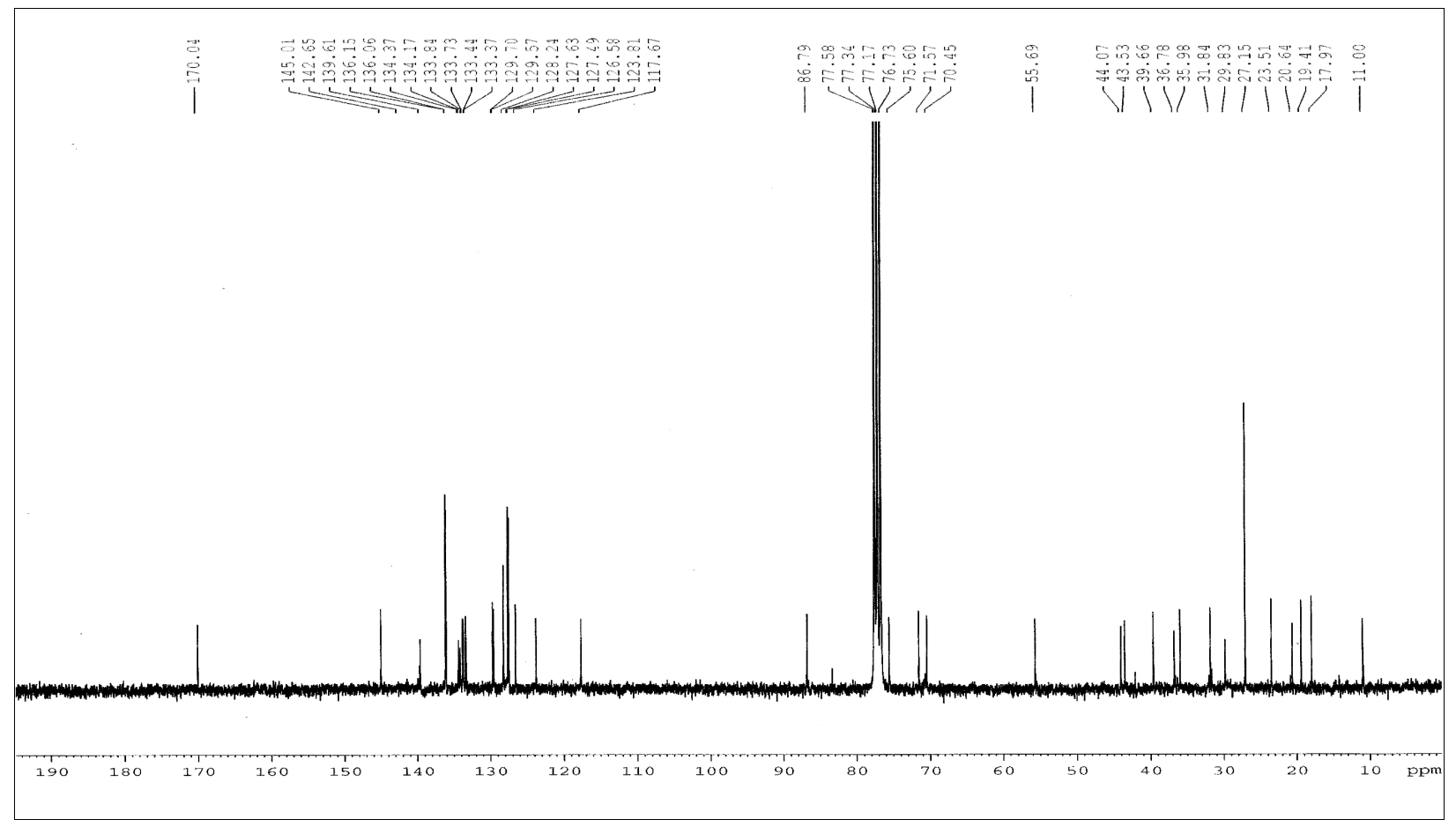

S36 
${ }^{1} \mathrm{H}$-NMR spectrum of Macrocycle $27\left(300 \mathrm{MHz}, \mathrm{CDCl}_{3}\right)$

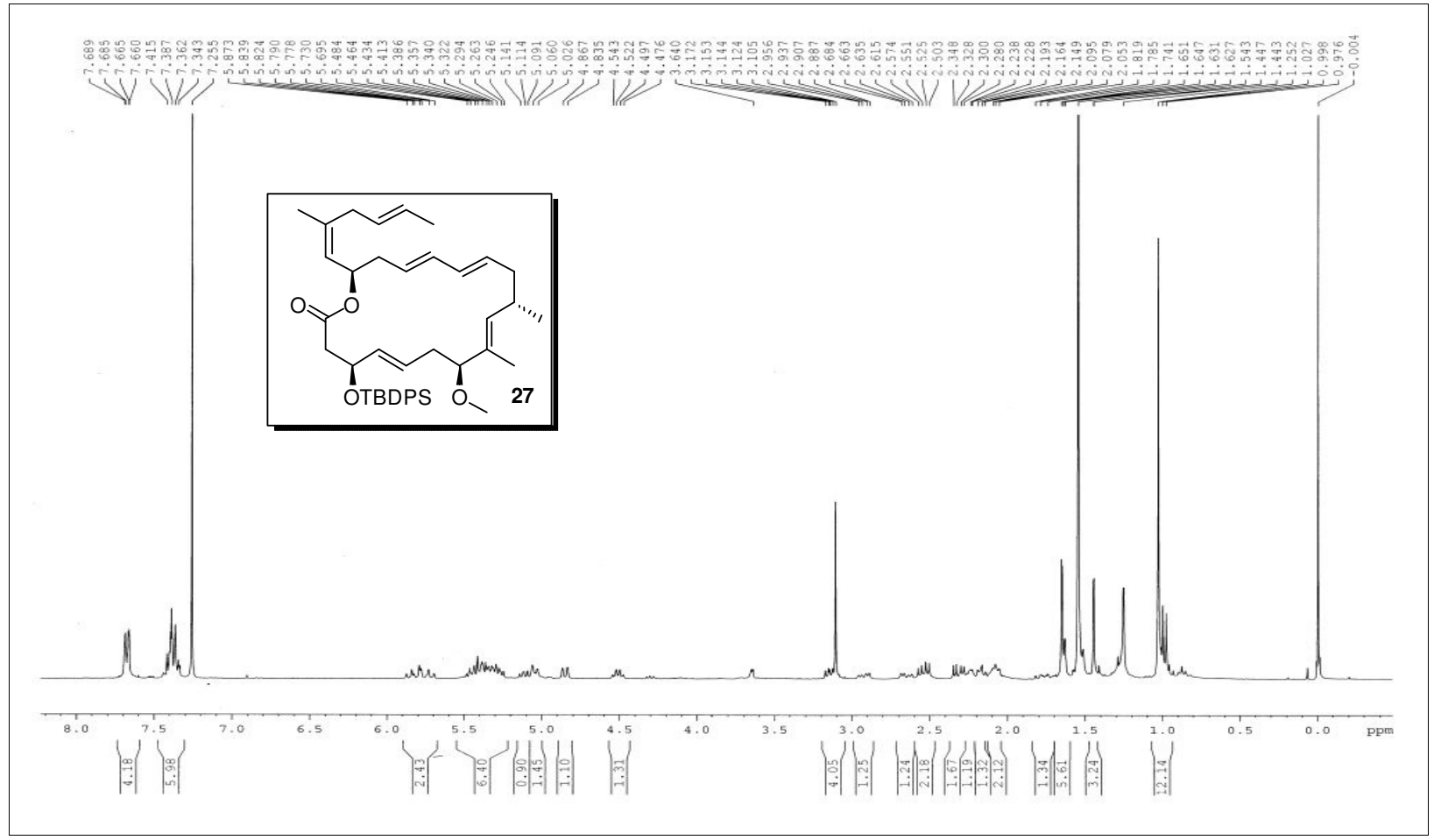

${ }^{13} \mathrm{C}$-NMR spectrum of Macrocycle $27\left(125 \mathrm{MHz}, \mathrm{CDCl}_{3}\right)$

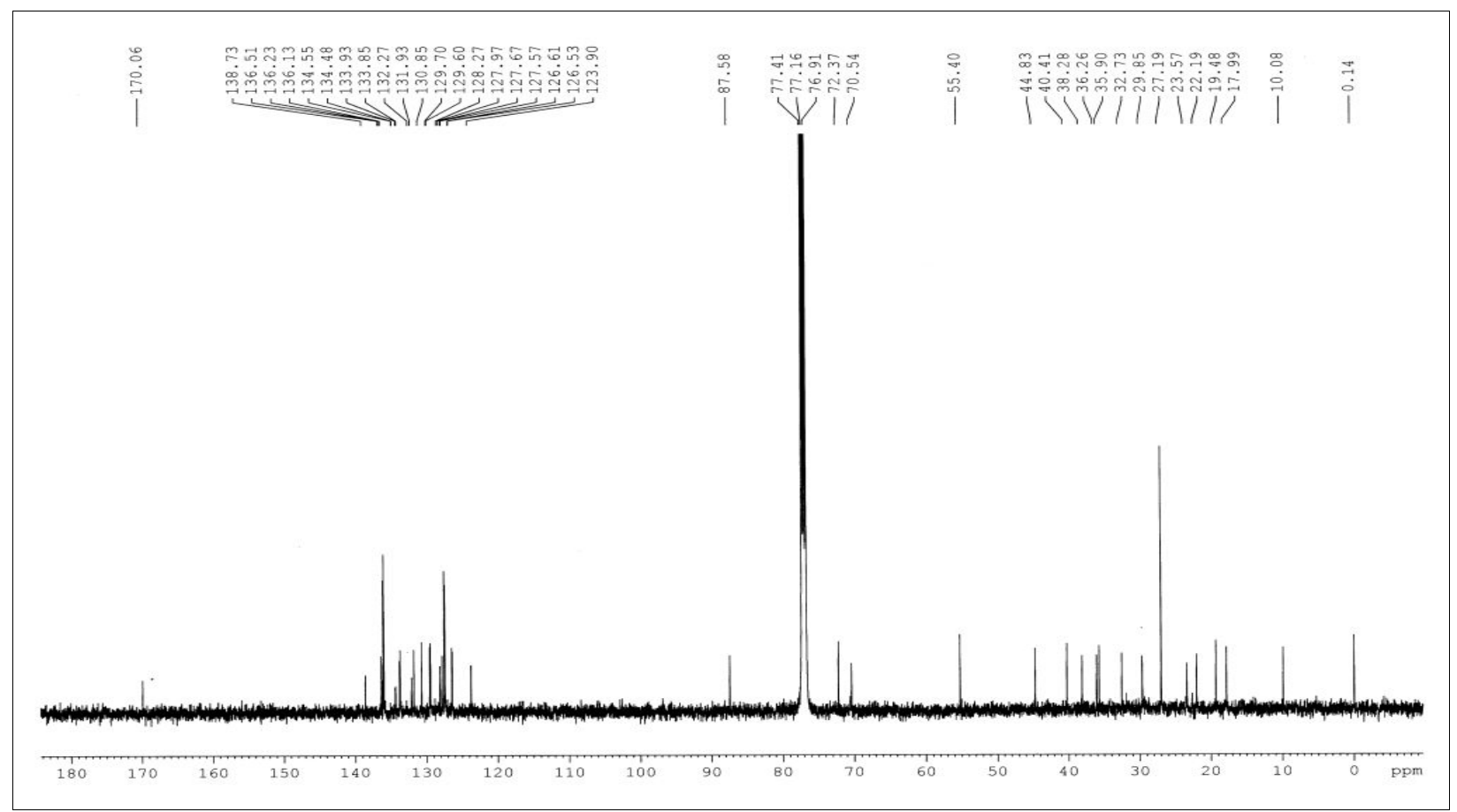

S37 
HSQC spectrum of Macrocycle $27\left(500 \mathrm{MHz}, \mathrm{CDCl}_{3}\right)$ :
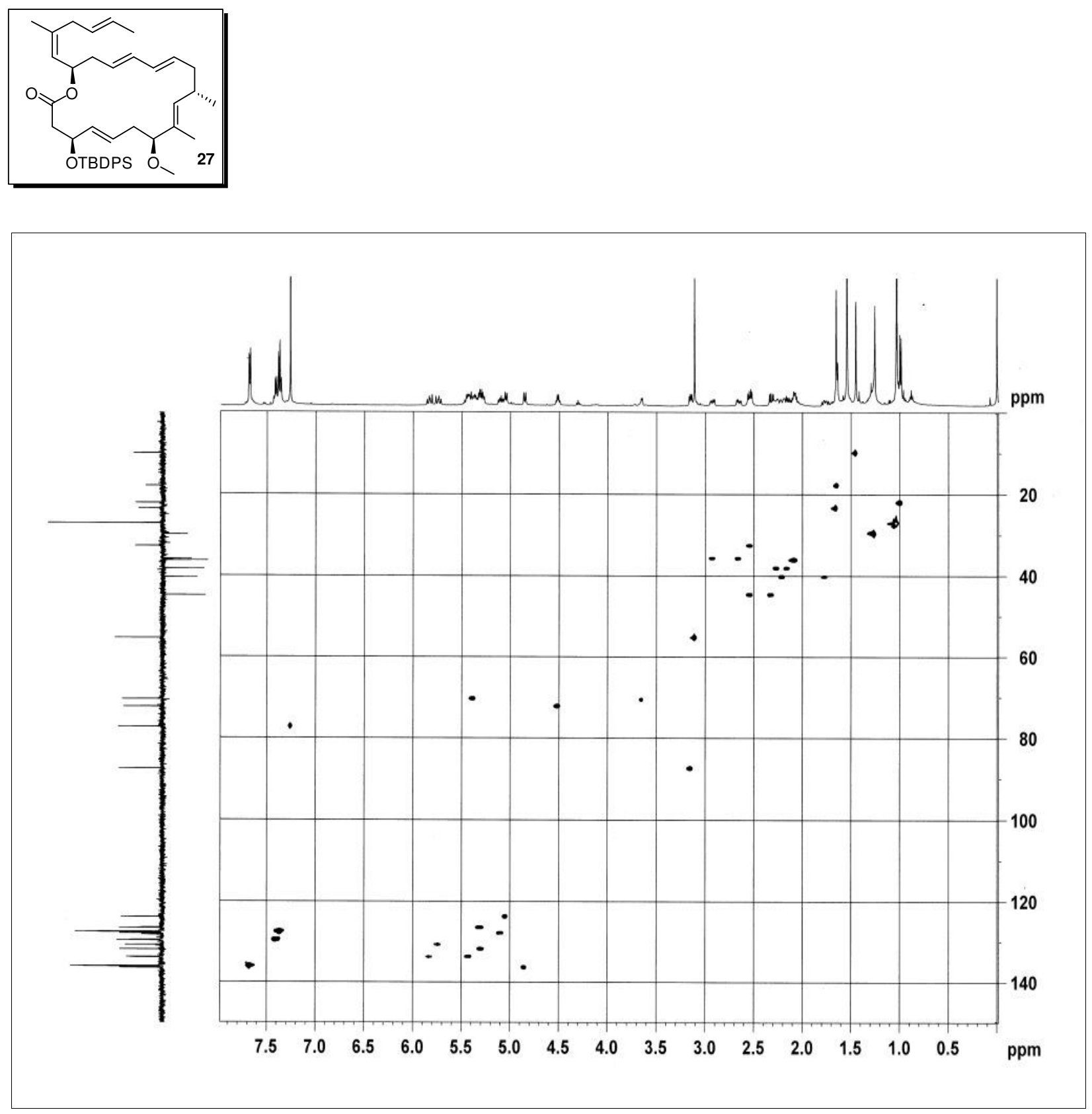
COSY spectrum of Macrocycle $27\left(500 \mathrm{MHz}, \mathrm{CDCl}_{3}\right)$ :
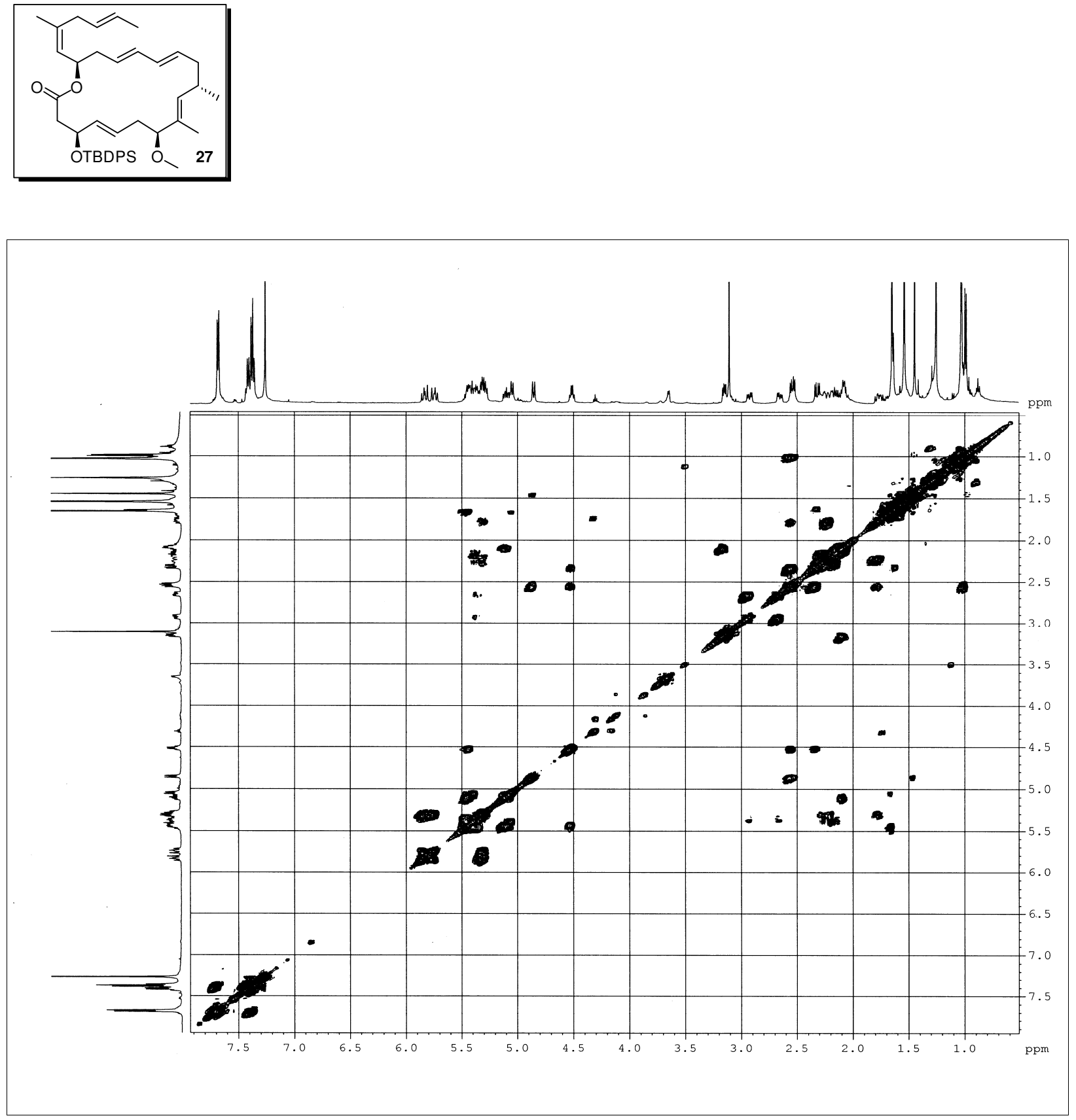
NOESY spectrum of Macrocycle $27\left(400 \mathrm{MHz}, \mathrm{CDCl}_{3}\right)$ :
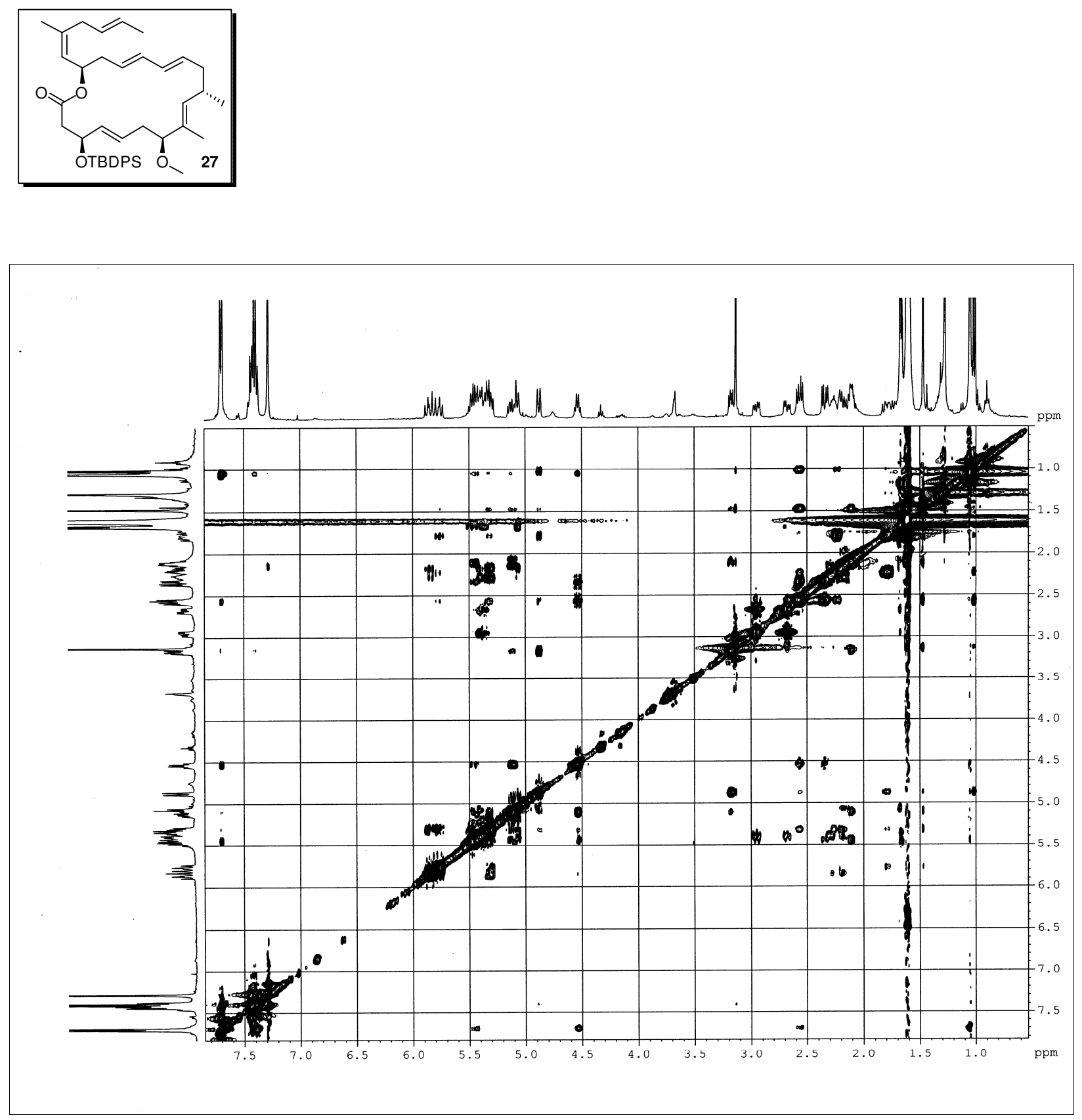
HMBC spectrum of Macrocycle 27 (125 $\left.\mathrm{MHz} \mathrm{CDCl}_{3}\right)$ :
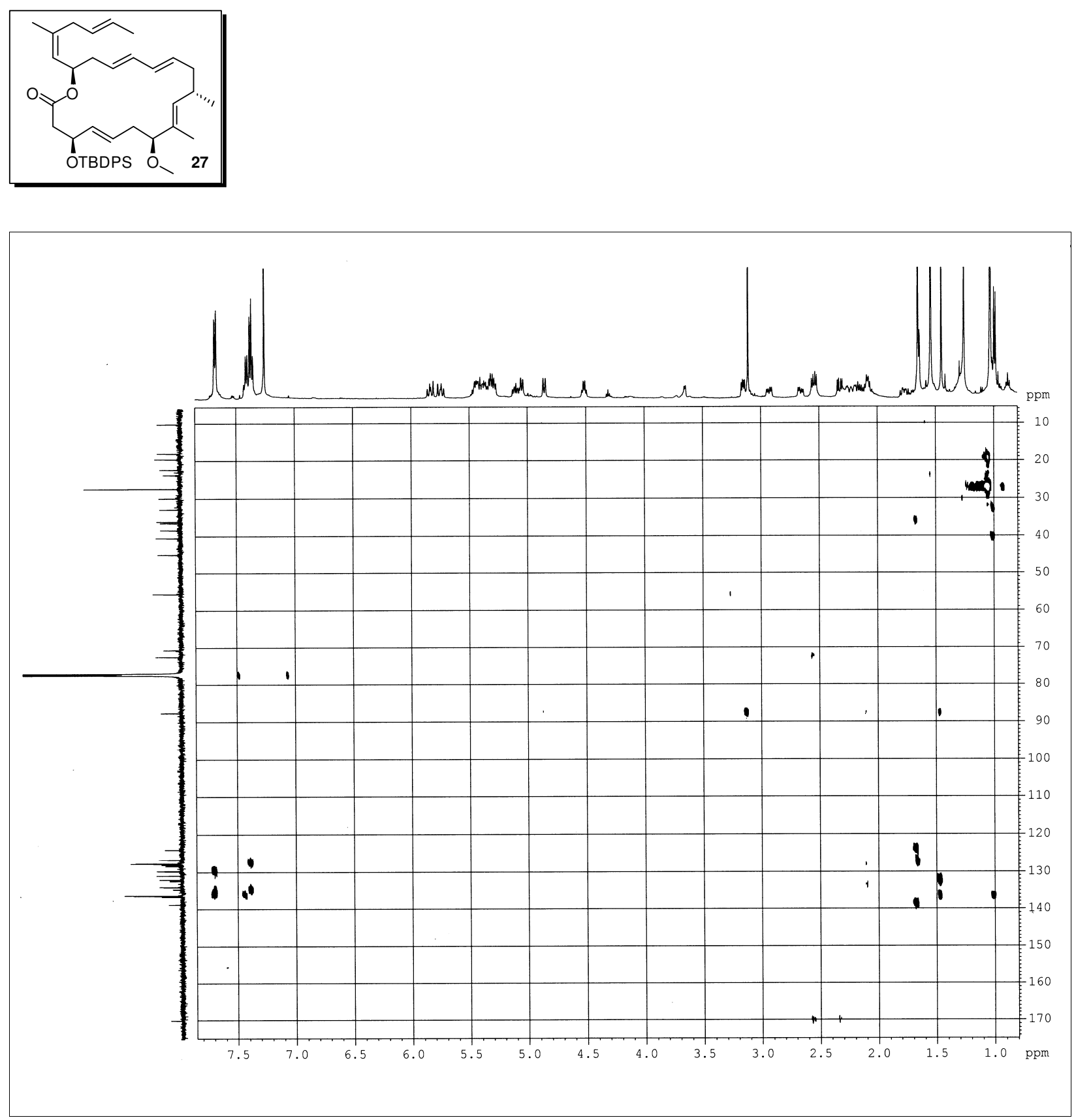
${ }^{1} \mathrm{H}-\mathrm{NMR}$ spectrum of Biselyngbyolide $\mathrm{B}(2)\left(300 \mathrm{MHz}, \mathrm{CDCl}_{3}\right)$

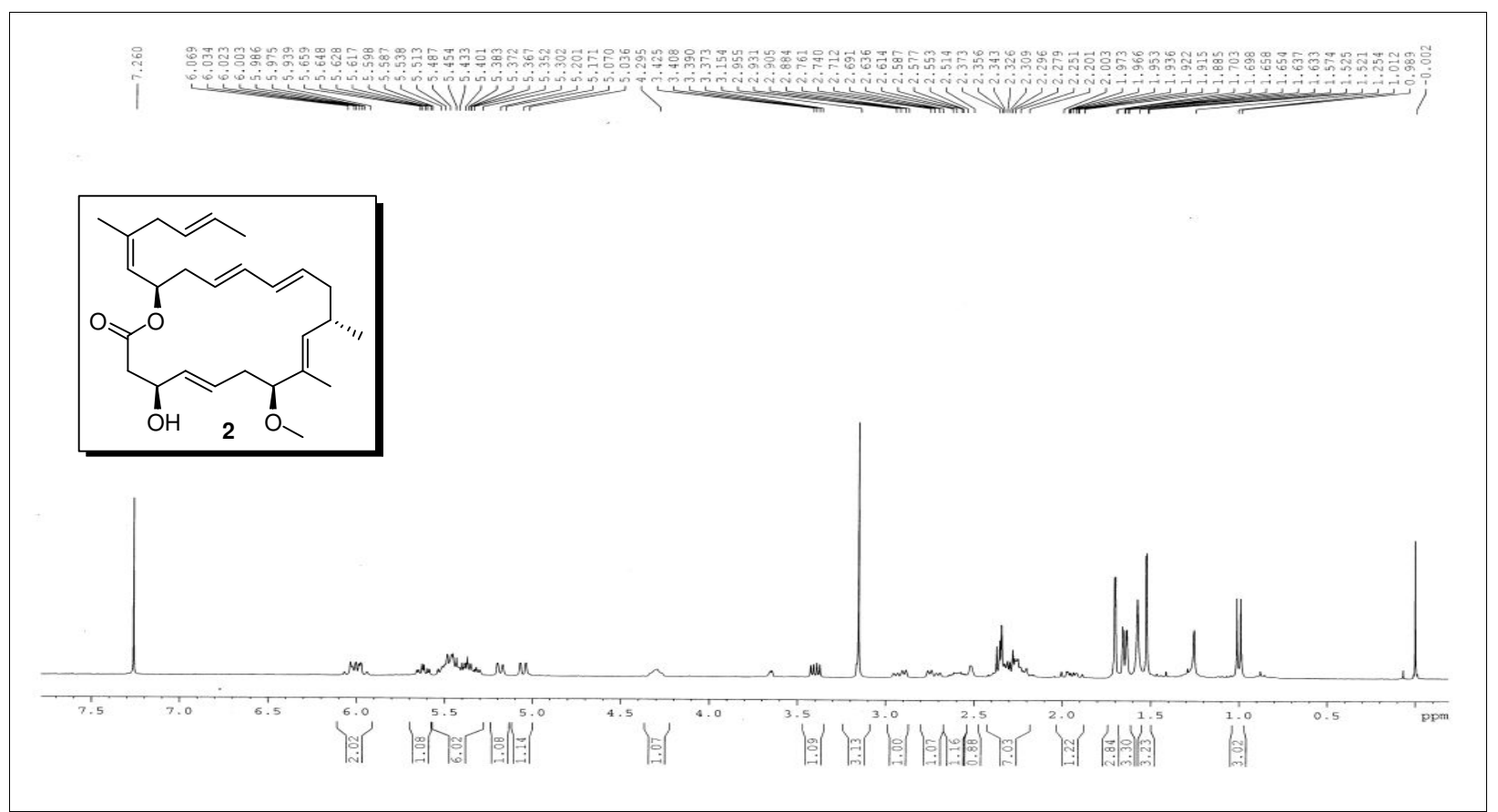

${ }^{13} \mathrm{C}-\mathrm{NMR}$ spectrum of Biselyngbyolide $\mathrm{B}$ (2) (75 MHz, $\left.\mathrm{CDCl}_{3}\right)$

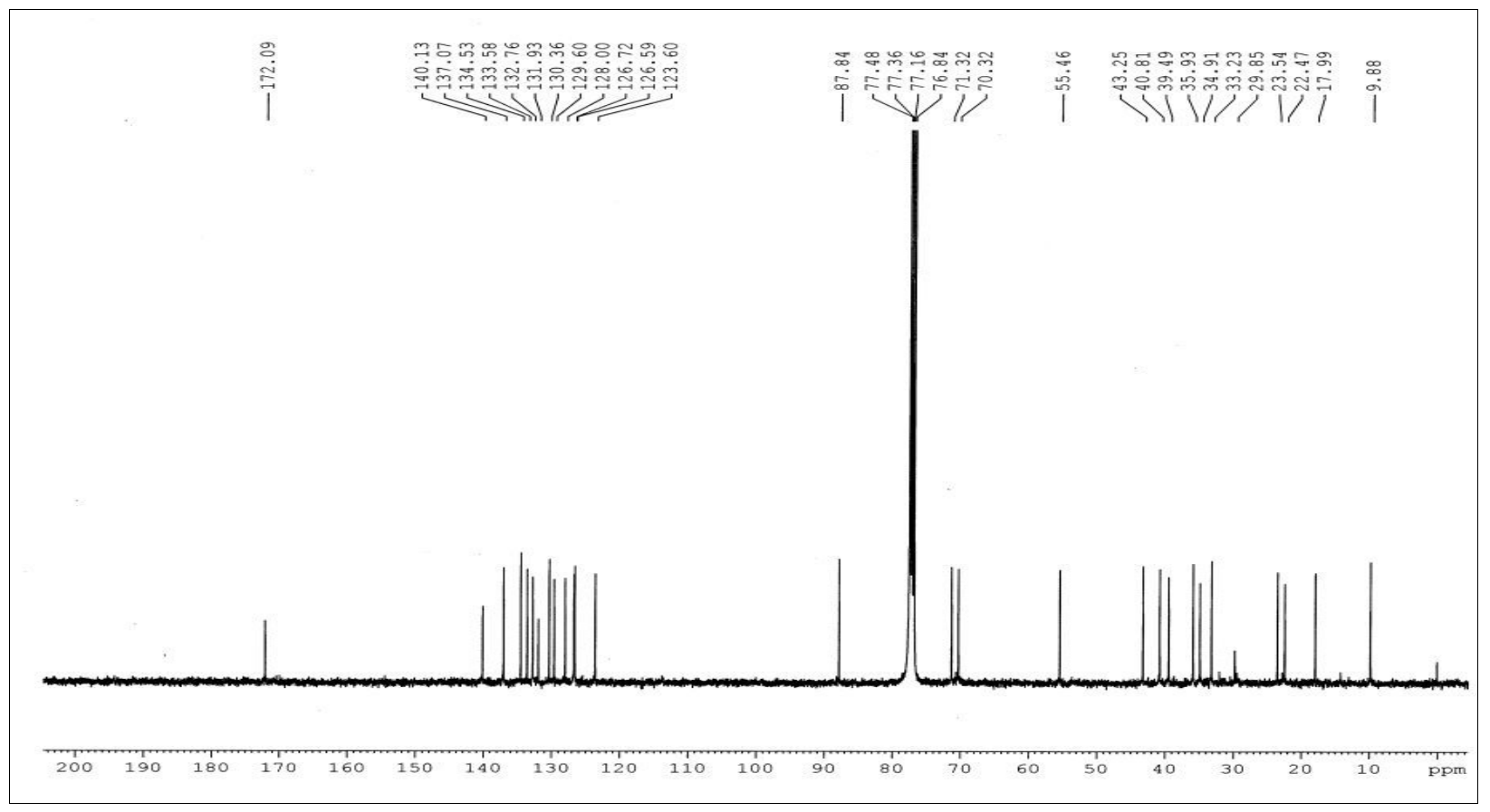


${ }^{1} \mathrm{H}-\mathrm{NMR}$ spectrum of Biselyngbyolide B (2) (300 MHz, $\left.\mathrm{C}_{6} \mathrm{D}_{6}\right)$

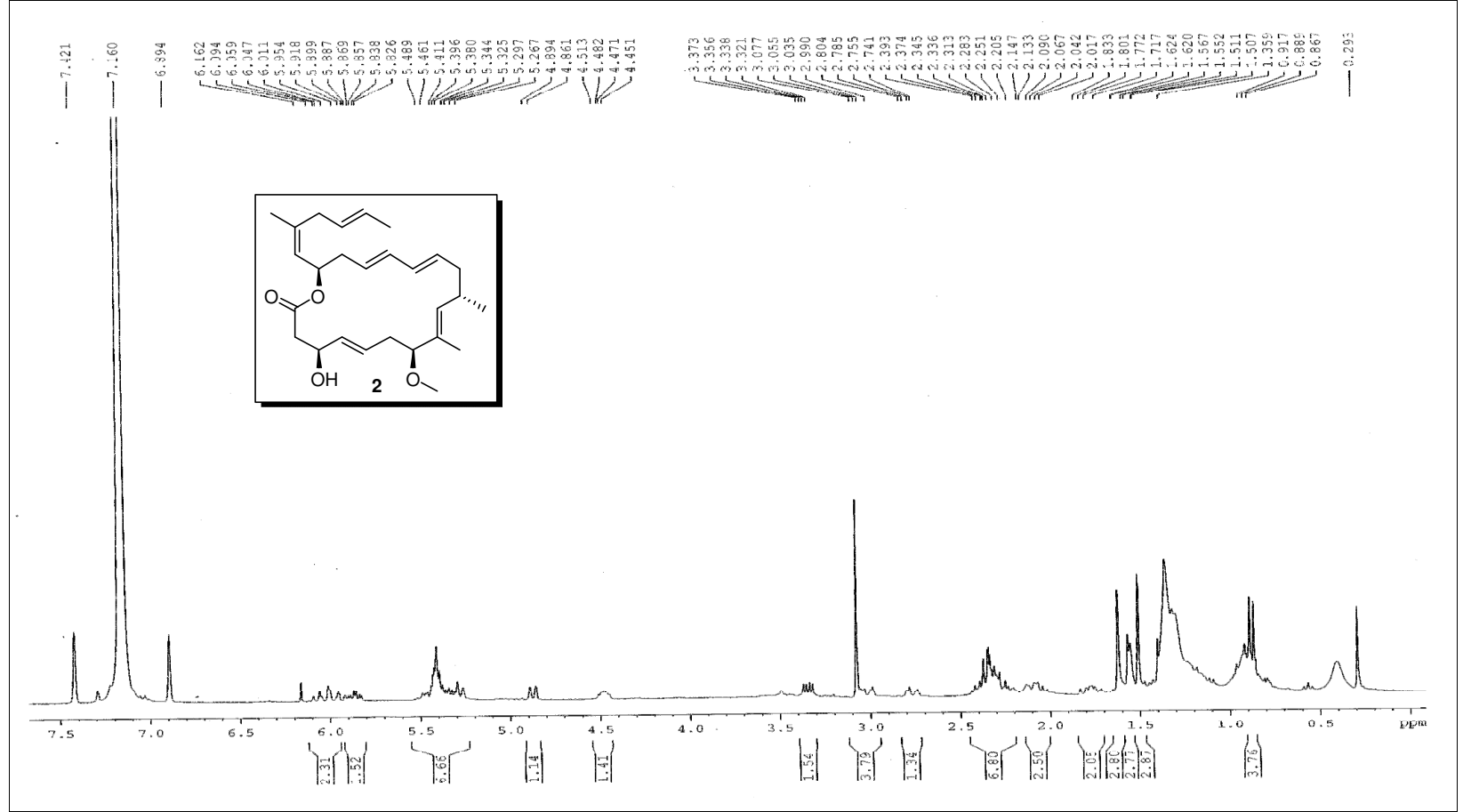

${ }^{13} \mathrm{C}-\mathrm{NMR}$ spectrum of Biselyngbyolide $\mathrm{B}(2)\left(125 \mathrm{MHz}, \mathrm{C}_{6} \mathrm{D}_{6}\right)$

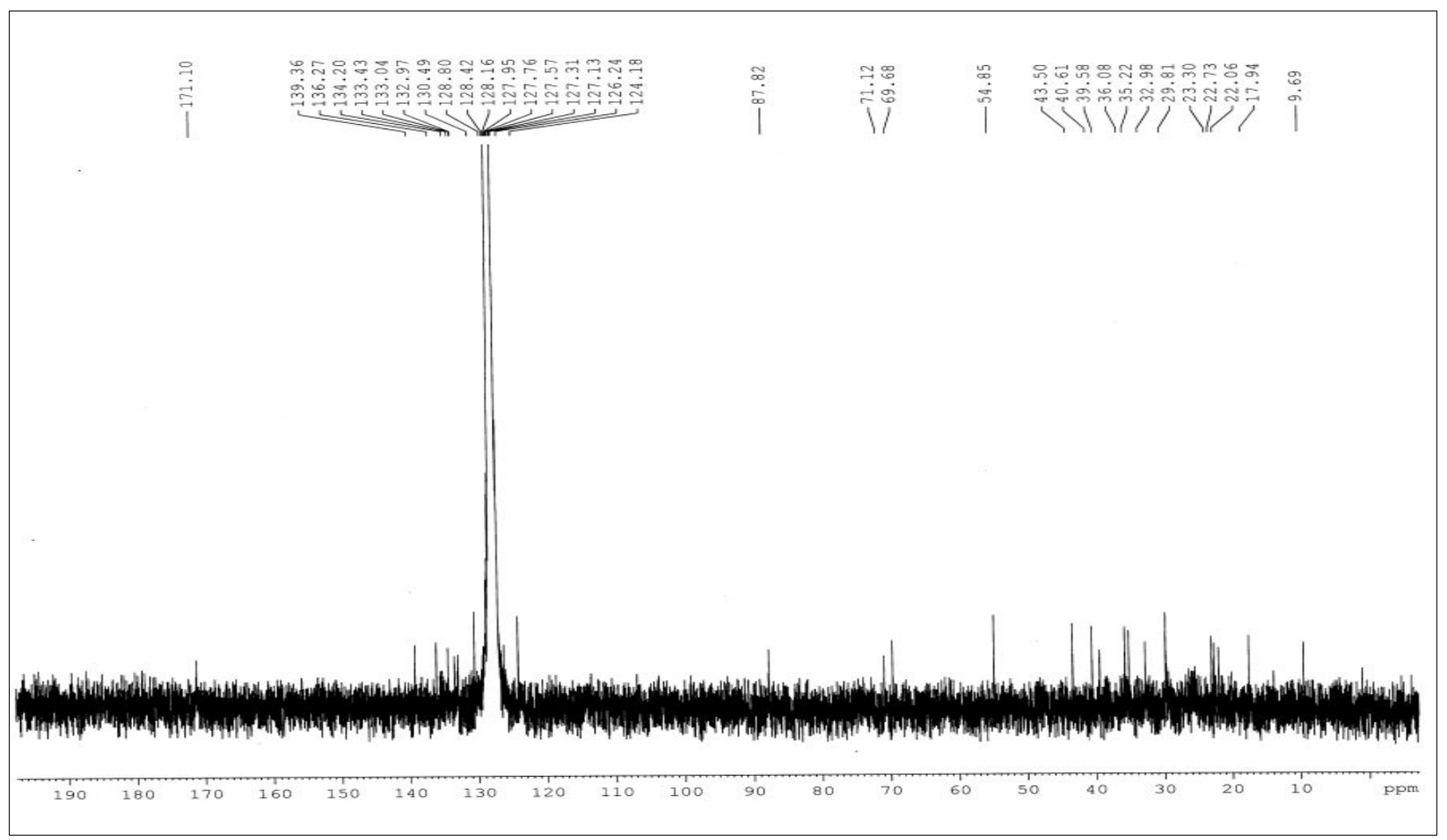


HRMS spectrum of Biselyngbyolide B (2)

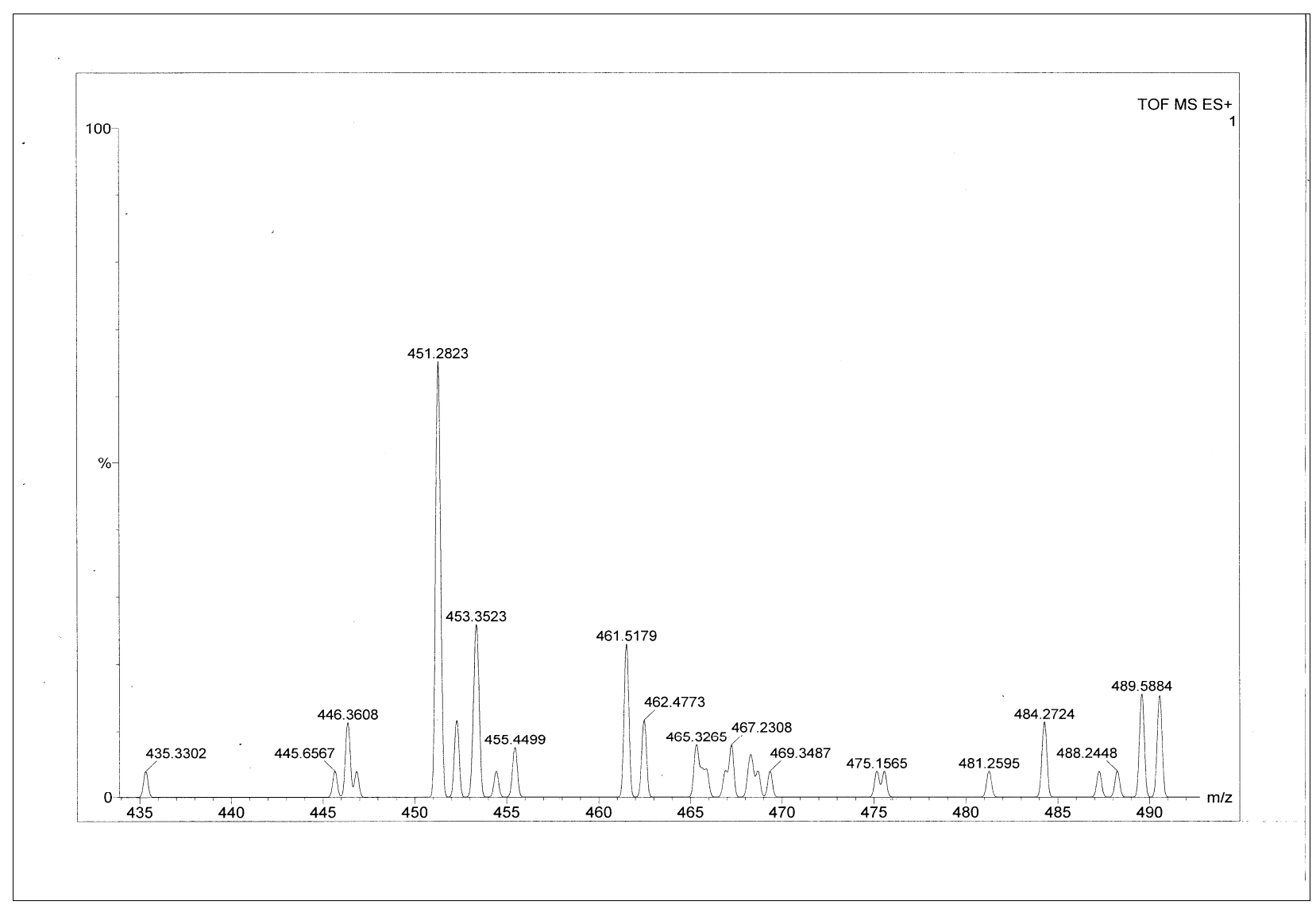

S44 